\title{
Processing Digital Images
}

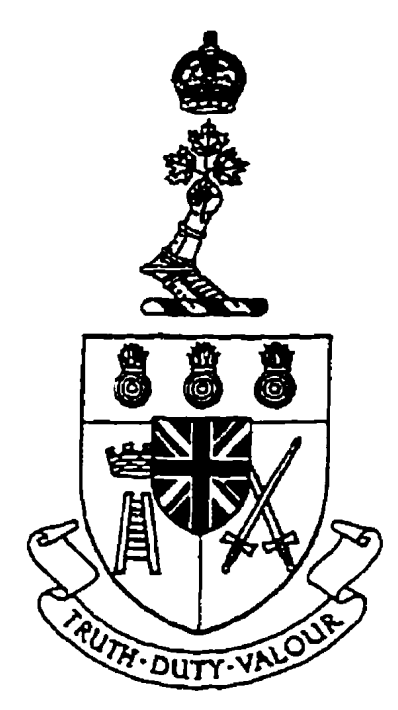

By

Captain John D.A. Bourne, BSc. (RRMC), CD

Canadian Armed Forces

A Thesis submitted to the School of Graduate Studies

in the Department of Mathematics and Computer Science

Royal Military College of Canada

Kingston, Ontario

In partial fulfilment of the requirements for The Degree of Master of Science in Computer Science

September 1996 
National Library of Canada

Acquisitions and Bibliographic Services

395 Wellington Street Ottawa ON K1A ON4 Canada
Bibliothèque nationale du Canada

Acquisitions et services bibliographiques

395, rue Wellington Ottawa ON K1A ON4

Canada
The author has granted a nonexclusive licence allowing the National Library of Canada to reproduce, loan, distribute or sell copies of this thesis in microform, paper or electronic formats.

The author retains ownership of the copyright in this thesis. Neither the thesis nor substantial extracts from it may be printed or otherwise reproduced without the author's permission.
L'auteur a accordé une licence non exclusive permettant à la Bibliothèque nationale du Canada de reproduire, prêter, distribuer ou vendre des copies de cette thèse sous la forme de microfiche/film, de reproduction sur papier ou sur format électronique.

L'auteur conserve la propriété du droit d'auteur qui protège cette thèse. $\mathrm{Ni}$ la thèse ni des extraits substantiels de celle-ci ne doivent être imprimés ou autrement reproduits sans son autorisation. 


\section{Dedication}

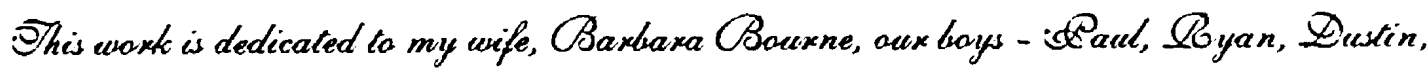
Daniel and SYCichael - and my mother for theix kind support, understanding and encouragement in the hursuit of my OTlaster's degree. 


\section{CAcknowledgements}

Qf is with genuine pleasure that $Q$ t recognixe the great assistance afforded me luy my

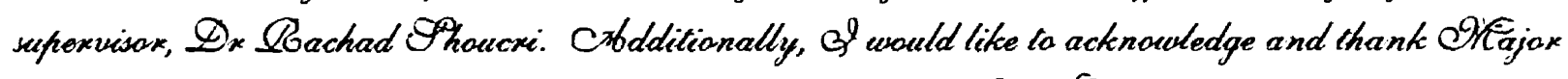

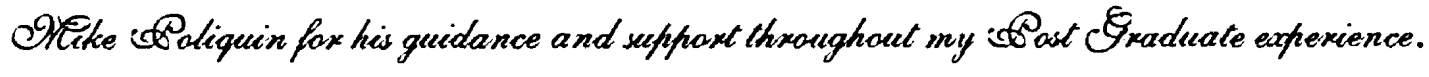


Qita

Qriginally from Ealgaxy, Crblherta, Eaptain Sthn David Crbndreun Bounne jained the Eanadian. Faxces in. Tuly of 1983. Abe shent the forst foux years of his ca reex completing a Bachelor's

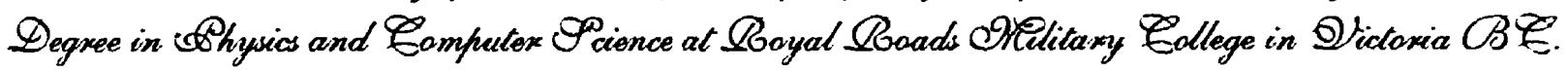

Crbftex completing his degree and the summex thase training required of an axmoux officex, Fecond-Dieutenant Bauxne was fasted to balgaxy to sexwe with an Crbxmoux Bogiment, Daxd Firathoona's Obaxse (Boyal Danadians). Duxing these three years he sexved with his Boegiment

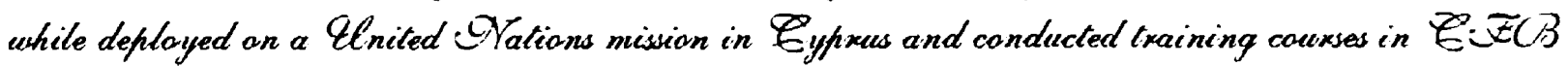
Gagetoun and EOB Baxden. Abe was promoted to Eaptain in OTay of 19.90.

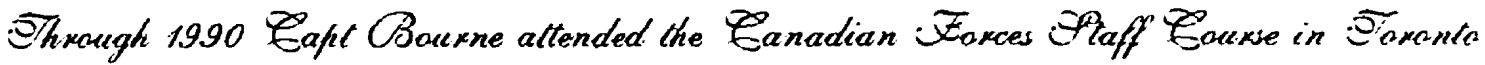
Qntario and was frosted to the Begulax Fuphart Flaff Officex pasition with the Faskatcheruen Dragoans in Mrose Vaus, Oaskatchewan.

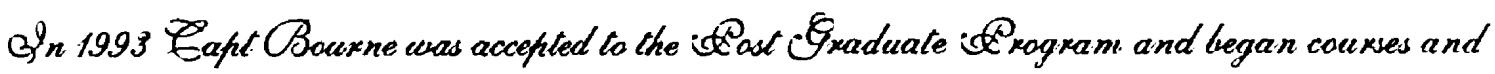

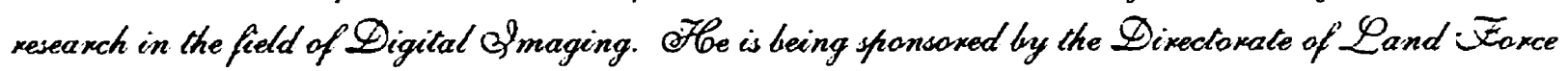
Development, STational Defence headquarters, Ottawa.

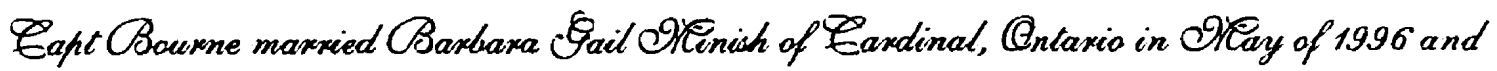
cogethex they are raising fure children - Drichaed, Daniel, Dustin, Ruyan and Baul.

v 


\section{Table of Contents}

\section{Opening Material}

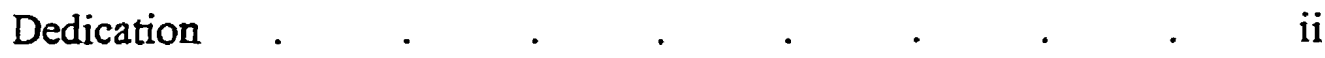

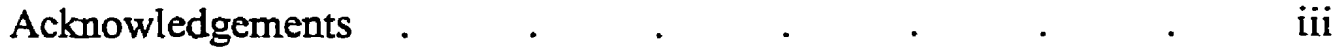

Vita . . . . . . . . . . . . . . .

Abstract

Table of Contents

List of Figures

Chapter One

Introduction

1-1 Opening Comments

1-2 Aim

1-3 Thesis Overview

1-3.1 Limitations to the Aim .

1-3.2 Implementation

1-3.2.1 Hardware used . . . . . . $\quad 6$

1-3.2.2 Software used $\quad$. $\quad$. $\quad$. $\quad$. $\quad 7$

1-3.2.3 Programming Approach . . . 7

1-3.3 Thesis Organization

Chapter Two

Theoretical Background - General

2-1 Introductory Comments .

2-2 TIFF format

2-3 M-mode Echocardiograms

2-4 Image Processing

2-4.1 General .

2-4.2 Unary Operations

2-4.3 Binary Operations 
Edge Detection - Theory

3-1 Introductory Comments . . . . . . . . 34

3-2 Properties of Edges . $\quad . \quad . \quad . \quad . \quad . \quad . \quad 35$

3-3 Overview of Edge Detection Techniques . . . . 38

3-4 LoG Filter . . . . . . . . . . . . 48

3-5 DoG Filter . $\quad . \quad$. $\quad . \quad$. $\quad . \quad$. 58

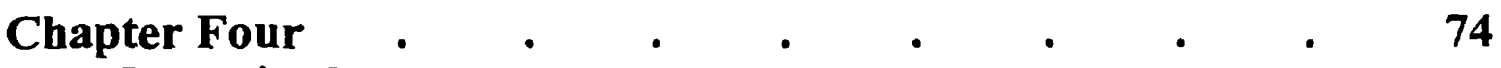

Image Processing Program

4-1 Introductory Comments . . . . . . . 74

4-2 Program Design and Listing . . . . . . 76

4-3 Processing Environment . . . . . . . . 77

4-4 Importing Images $\quad . \quad$. $\quad . \quad$. $\quad$. $\quad . \quad 85$

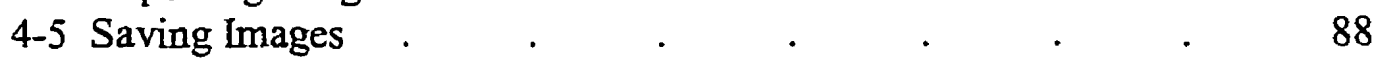

4-6 Deleting Images . $\quad . \quad$. $\quad$. $\quad$. $\quad$. $\quad$. 91

4-7 Image Processing 93

4-7.1 Add . $\quad . \quad$. $\quad . \quad$. $\quad . \quad$. $\quad .93$

4-7.2 Brightness . . . . . . . . . $\quad$. 94

4-7.3 Crop . $\quad . \quad$. $\quad . \quad . \quad . \quad . \quad .95$

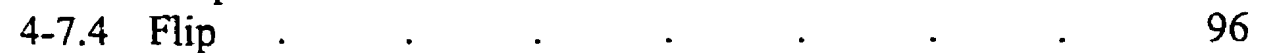

4-7.5 Histogram Operations. $\quad$. $\quad$. $\quad$. $\quad$. 99

4-7.6 Multiply . . . . . . . . 102

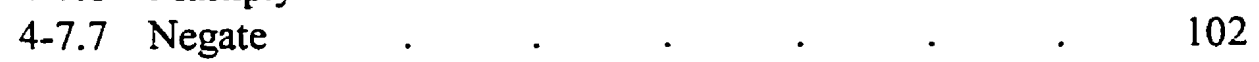

4-7.8 Subtract . . . . . . . . . 103

4-7.9 Trim . . $\quad . \quad . \quad . \quad . \quad . \quad . \quad 103$

$4-7.10$ Zoom . $\quad . \quad . \quad . \quad . \quad . \quad . \quad . \quad 104$

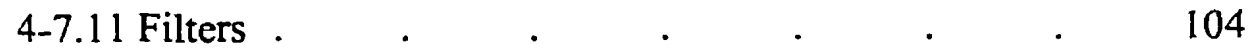

4-7.11.1 Median . . . . . . . 104

4-7.11.2 Smoothing . . . . . . 105

4-7.11.3 Derivative of a Gaussian . . . . 105

4-7.11.4 Laplacian of a Gaussian . . . . 106 


\section{Chapter Five}

Experimentation and Results

5-1 Introductory Comments .

5-2 Experimental Results Using the LoG Filter . . . 111

5-3 Experimental Results Using the DoG Filter . . . 118

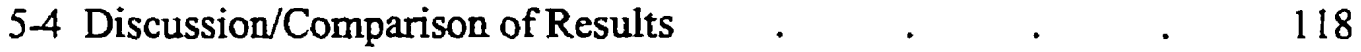

\section{Chapter Six}

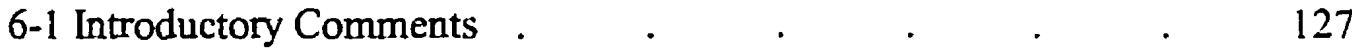

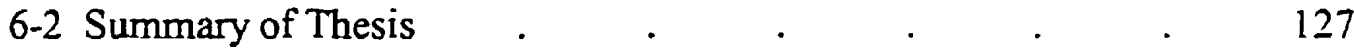

6-3 Recommendations for Future Research . . . . . 130

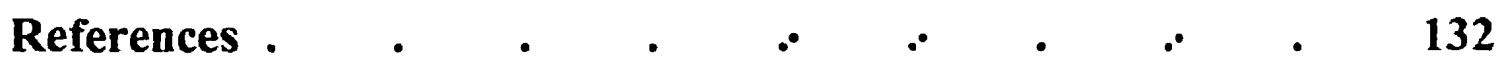

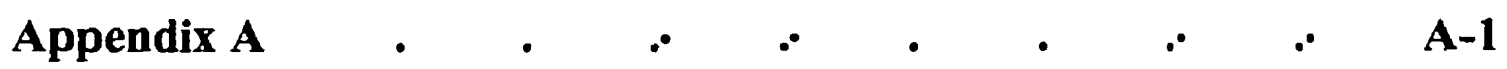
List of $\mathrm{C}++$ Drivers

Appendix B

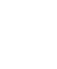

List of $\mathrm{C}++$ Libraries $\dot{\text { Used by the Program }}$

Appendix C

List of Processing Operations

Appendix D

List of Common Tags and Data Types Used in TIFF 


\section{List of Figures}

Number Title Page

2-I IFH, IFD, IFD Entry and Standard TIFF File Layout 15

2-2 The Digitization of an Image 22

3-1 Examples of Step Edge 36

3-2 Example of Transitional Edge and Noise Effects 37

3-3 Edge Classification Problem 38

3-4 Effects of Low Pass and High Pass Filters 40

3-5 Sharpening Using a Low Pass Filter 41

3-6 Examples of First derivative Filters 42

3-7 Image Selected for Edge Detection Example 54

3-8 Convolution Kernel for LoG with $\sigma=1.0$ and $M=9$

3-9 Example of LoG Convolution 56

3-10 Normalized Pixel Values after LoG Filtering 57

3-11 Convolution Kernels for DoG with $\sigma=0.5$ and $\sigma=0.8 \quad 61$

3-12 DoG Search Paths 62

3-13 $n_{1}$ : Result after Convolution of Example with $G_{x}$

3-14 $\mathrm{n}_{2}$ : Result after Convolution of Example with $\mathrm{G}_{\mathrm{y}} \quad 66$

3-15 Amplitude Array for Gaussian Smoothed Image Gradient, 67 Based on $\mathrm{n}_{1}$ and $\mathrm{n}_{2}$

3-16 Angle Array for Gaussian Smoothed Image Gradient, 68 Based on $\mathrm{n}_{1}$ and $\mathrm{n}_{2}$

3-17 Array of all Maxima Encountered $\quad 69$

3-18 Array of all Minima Encountered 70

3-19 Initial Array of Edge Points (where angles match) 71

3-20 Final Edge Map after Gap Closing Efforts 72

4-1 Program Workspace $\quad 78$

4-2 Image Processing Program Command Set 81

4-3 Image Display and Movement When One or Two Images are 83 Displayed in the DISPLAY_AREA

4-4 Example of Autoreduction 86

4-5 Example of Histogram Window 98

4-6 Example of Histogram Preview Option 101

5-1 Test Image of M-mode Echocardiogram 109

5-2 TIFF Information for Test Image 110

5-3 Normalized Gray Scale Result after LoG Filtering ( $\sigma=1.0) \quad 112$ 
5-4 Black and White (+ve/-ve Regions) Result after 113

$$
\text { LoG Filtering }(\sigma=1.0)
$$

5-5 Edge Map Derived from LoG Filtering $(\sigma=1.0) \quad 114$

5-6 Edge Addition of Figure 5-5 to Original Test Image $(\sigma=1.0) \quad 115$

5-7 Edge Map Derived from LoG Filtering $(\sigma=2.0) \quad 116$

5-8 Edge Map Derived from LoG Filtering ( $\sigma=3.0) \quad 117$

5-9 Edge Map Derived from DoG Filtering $(\sigma=0.5) \quad 119$

5-10 DoG Edge Map Thresholded with all Contours Set 120 to Black ( $\sigma=0.5)$

5-11 Edge Map for Figure 5-9 Thresholded (lower) at Gray 121 Level $20(\sigma=0.5)$

5-12 Addition of Figure 5-11 ( $\sigma=0.5)$ to Original Image 122

$5-13$ Edge Map Derived from DoG Filtering $(\sigma=1.0) \quad 123$

5-14 Thresholding of Figure 5-13 in Black $(\sigma=1.0) \quad 124$

$5-15$ Subtraction of LoG Images with $\sigma=3 \& \sigma=2 \quad 125$ 


\section{Chapter One}

\section{Introduction}

\section{1-1 Opening Comments}

Of the five senses, probably the most powerful and most relied upon is vision. Being able to see images of the world provides a vast expanse of information on what is before a person and where it is located [Marr82]. Various tools have been developed over the ages to improve man's ability to capture and exploit this information such as the easel, telescope, microscope and camera. With the onset of the information age newer more sophisticated technoisgies bave appeared such as $\mathrm{x}$-ray machines, radar, sonar, electron microscopes, thermal imagery, computed tomography scans, and magnetic resonance imagery. All of these devices share the common trait that they are tools designed to broaden or deepen man's perspective within this world and beyond.

In modern society, the computer has emerged as a powerful new tool in the processing of the mass of visual and 2-dimensional information provided by sources such as those mentioned above. This information is passed to the computer in the form of a digital image, which is a discrete array of optical densities or intensities represented by integers. The processing and enhancement of digital images affects a broad range of scientific applications such as 
engineering, design, weather analysis, geographical analysis, weapon design, non-destructive testing, astronomy, robotics, topography, photography, medical analysis, nuclear physics, and a host of other areas [Ekst84]. Indeed, any discipline which uses information derived from optical, acoustical or electro-magnetic sensors can benefit from some degree of image processing.

To date, it has been difficult to exploit this new realm of technology due to hardware constraints. For example, to store an image which is 1000 pixels wide by 1000 pixels in height using 164 gray scales takes 1 megabyte or eight million bits of memory. Most processing techniques sample each pixel of the image, often multiple times, so that for an image containing $n$ pixels, the process may take from order $n$ operations to order $n^{2}$ operations or higher to execute. Owing to their great magnitude and hardware limitations, such processes often took hours to complete. Today's computer systems are much better equipped to handle the scale of these operations. Storage devices capable of storing over one gigabyte of information are now common and have much faster acquisition (read/write) times than previous devices. Also, very large scale integration (VLSI) technology is speeding up the rate at which information is transferred and processed within the computer as has the addition of integrated math coprocessors. Additionally, although the vast majority of computers today are still based on the classic Von Neumann design principles (sequential data processing), considerable research is being conducted into parallel processing architectures which are often naturally suited to algorithms used in many applications [Akl89]. Parallel processing breaks down a problem into many smaller parts, each of which is solved simultaneously by a different processor. Examples of problems suited to parallel processing include search, sort, and combinatorial or numerical 
problems - which include image processing functions such as point processes and convolution. As parallel processing machines become more advanced and available, they can have the effect, in these areas, of reducing order $\mathrm{n}(\operatorname{logn}), \mathrm{n}^{2}$ or higher order operations down to as low as order n operations [Akl85]. All of these factors are reducing the time required to conduct image processing down to reasonable levels and, in many cases, allowing real time analysis. Finally, in concert with this, advances in video technology have allowed much more intricate and accurate displays of the original images and the results of processing [Ekst84].

Once an analog signal is digitized, a vast number of processes are available to manipulate the pixels, which define the image, in order to improve the information provided to the user. The results of such processes are generally an enhancement of the image in some manner or a restoration of the image after some form of degradation. Some basic processes include magnification, rotation, and linear brightening. Going further, an image may be thresholded or equalized in order to accentuate certain intensities in the image (contrast enhancement). As a degree of noise is introduced whenever an image is acquired via optical or electronic means, another form of image enhancement is the reduction of the degrading effect of this noise. Other forms of restoration include deblurring and interpolation. An image may also be analysed using various matbematical techniques to accentuate certain aspects of the image such as contour detection, texture analysis, motion analysis and scene analysis.

This world of machine vision is generally thought of in two levels - low level vision and high level vision. Low level vision includes all the processes wherein a basic image is altered in order to improve the quality or representation of the image [Ekst84]. High level vision involves the analysis of an image to determine content and involves such operations as pattern 
recognition, texture analysis, and scene analysis [Chel85].

\section{1-2 $\underline{\text { Aim }}$}

The aim of this research has been to explore aspects of low level vision such as image storage, display and enhancement as well as develop a user-friendly software package in the $\mathrm{C}$ language capable of performing these functions. The application program's operations were to range from point processes such as negation, orientation changes, addition of two images. subtraction of images, histogram techniques and brightening to area processes used in algorithms such as median filtering and edge detection.

This research is intended to augment the work of the thesis advisor, Dr Rachad Shoucri in his study of M-mode Echocardiograms. As such, edge detection and enhancement was selected as the primary application and the techniques explored in this research are applied to digitized representations of M-mode Echocardiograms to test their effectiveness and usefulness in this field.

\section{1-3 Thesis Overview}

\section{1-3.1 Limitations to the Aim}

A number of limitations have been adopted in order to confine the scope of our work to a realistic subset of the image processing/analysis domain. To begin with, there are a number 
of methods for storing images in memory including:

TIFF - Tagged Image File Format;

GIF - Graphics Interchange Format:

Bmp - Bit map used by OS/2, MSWindows;

PCX - Used by PCPaintbrush and others;

TGA - Targa file format; and

EPSF - Encapsulated post script.
[Lind91][Luse93]

[Liaw95]

[Lind91]

In our case, the TIFF format was selected primarily owing to its widespread acceptance in the field [Lind91][Luse93]. Nonetheless, new modules could be added with relative ease to allow the application program to import and use images saved in any of the above formats.

It was also decided that compressed files would not be supported in our application. There is a compressed TIFF format (in TIFF 5.0); however, compression algorithms were considered beyond the scope of this research and as such were not implemented. Nonetheless. it is possible to add a module to the existing program to read and write in compressed formats if considered necessary at some future date.

Thirdly, the program was developed on an Intel machine and, at present, supports TIFF's Intel format but does not support images stored in TIFF's Motorola format; although this too could be readily added as necessary (see section 2.2 for more information on TIFF). In any case, the application program itself is designed for use on an Intel platform and has not been tested on a Motorola system.

The final restriction adopted has been to confine images to gray scale only ( 256 levels). This has been done for two reasons. First, M-mode Echocardiograms are displayed in gray scale and, as they are the focus of the application, development in gray scale was considered the first priority. Secondly, this restriction was adopted in order to allow sufficient opportunity to explore 
a number of different techniques for the enhancement of the M-mode images, particularly as there is a great deal more information available on research into algorithms suitable for gray scale images than for colour images.

\section{1-3.2 Implementation}

\section{1-3.2.1 Software:}

The program developed as part of this thesis was written in the $C$ programming language. It was written and compiled using Borland $\mathrm{C}++$ version 3.1 [Borl92-1 - Bor192-4] and uses Borland $C++$ class libraries. Later versions of $C++$ such as Borland's version 4.5 are suitable for editing; however, it is not possible to run the application program in a Window's environment as Borland $\mathrm{C}++4.5$ does not support the graphics mode used by version 3.1 [Fais91]. The graphics drivers required to run the program are listed in Appendix A. The overall program was designed as a project wherein most functions are independently compiled and linked to the main program (main.c) prior to program execution. Within the functions which were developed for the thesis are many standard C+ library routines (for example "sprintfo"). The various standard routines offered by Borland $\mathrm{C}++[$ Borl92-1] are grouped into libraries of related routines. This necessitates the inclusion of the libraries which contain any of the standard routines used within the application program. A list of the standard libraries which were used is contained in Appendix B. Finally, a summary of the processing operations which can be done by the program - including the name of the applicable program function and a thesis page reference wherein an explanation of the operation can be found - is provided as Appendix C. 


\section{1-3.2.2 Hardware:}

The application program was developed using an Intel based Pentium $90 \mathrm{MHZ}$ computer with a monitor used in the VGA mode. The computer contained $16 \mathrm{MB}$ of RAM and a $500 \mathrm{MB}$ hard drive. Performance details provided are based on this architecture. The application program requires an Intel 286 or better, $640 \mathrm{~KB}$ of RAM and an EGA, CGA or VGA monitor. In addition, a Hewlett Packard ScanJet IIc scanner was used to digitize all of the images presented in this work.

\section{1-3.2.3 Programming Approach:}

The application program contains over 9000 lines of code and its executable file takes approximately $300 \mathrm{~K}$ of memory. It was designed using a modular approach called top-down design. Top-down design is the step-wise refinement of a large program designed to aid the reader in following the flow of the program as easily as possible and is based on the idea of "divide and conquer" [Mill87] [Hext90]. The overall program is designed such that the flow runs from top to bottom, the general to the specific, where small sub-tasks are at the leaves of a program flow tree [Ling79]. At each stage, a new module was added ("divide") using a "program stub" [Ling79] and then developed, refined and tested ("conquer") prior to the addition of the next capability. As often as possible, redundant code was avoided by creating smaller routines to handle common "housekeeping" tasks and having all larger modules call on these when required. For example, a module was created to print error messages to the screen whenever an error in input or program execution occurs. Another example is the function bello which is called whenever the program wants the computer to sound a bell to alert the user to 
some message being displayed. The overall task is thus decomposed into sub-tasks; which can in turn be further decomposed into sub-sub-tasks and so on. This forms a program hierarchy. which is displayed in section 4.21 within the application program chapter. Note that modules designed for use by other modules in "housekeeping" tasks are not displayed, rather, only those modules which are part of the user's command execution are included. As a result of the use of top-down design, new modules and/or improvements to existing modules can be done with relative ease as related modules are easily located and the program flow is readily identifiable.

\section{1-3.3 Thesis Organization}

This work has been broken down into five additional chapters. Chapter Two provides the foundation of the theoretical background required for the research. It begins with a more detailed explanation of the Tag Imaged File Format (TIFF) used by the application program in the retrieval and storing of images. Additionally, some of the basic theory behind M-mode Echocardiography is presented as background information for the discussion on the results of the image enhancements done on a digitized image of an M-mode Echocardiogram. Finally, background is provided into the different aspects of image processing which are applied in the program including both unary and binary operations. Chapter Three expands the theoretical background for the research by providing detail on edge detection theory. First, the general properties of edges are discussed, including a review of some of the classic spatial domain techniques which detect edges by looking at the discrete mathematical function of digitized images. This is followed by a detailed look at two techniques used to find edges in gray scale images: the Laplacian-of-a-Gaussian (LoG) filter and the Derivative-of-a-Gaussian (DoG) filter. 
Chapter Four provides details on the application program and the algorithms used to implement the various unary and binary operations discussed above, as well as the two edge detection techniques. It also discusses the overall capabilities/limitations of the program. Chapter Five provides example results from the use of the edge detection techniques on an M-mode Echocardiogram image as well as some concurrent examples of some primary program operations (unary and binary). It ends with a discussion and comparison of the results. The final Chapter, Six, provides the concluding material, including a review of the lessons which were leamed through the research and recommendations for future work in this field.

At the end of the thesis is a list of references used and a number of appendixes which are included to provide additional background material. Appendix A provides a list of $\mathrm{C}++$ drivers used to run the application program. Appendix B contains a list of the $\mathrm{C}++$ standard libraries used by the application program. Lastly, Appendix D provides a list of TIFF data types and some common TIFF tags. 


\section{Chapter Two}

\section{Theoretical Background - General}

\section{2-1 Introductory Comments}

This chapter provides an overview of some of the general background theory for the research. To begin with, the Tagged Image File Format (TIFF) will be explained in detail as the application program has been developed using this format exclusively to date. This will be followed by some background on M-mode Echocardiograms to aid in appreciating the results derived from applying some image processing techniques to such images. The last section of the chapter explores numerous low-level image processing techniques. It begins with an explanation of the categories of processes that are done on digitized images within the spatial domain - point, area, algebraic and geometric. A number of specific operations are then examined which fall into two basic groupings: unary and binary operations. Unary operations include such procedures as cropping, negation, scaling, adjusting brightness, histogram techniques, and median filtering. Binary operations include addition, subtraction and multiplication of images. The next chapter will then explore more intricate processing techniques related to the detection of contours. 


\section{2-2 TIFF Format}

In order for a machine to display and manipulate an image, certain key questions need to be answered. For example:

1. What are the width and height of the image?

2. Is the image colour, bi-level or gray scale?

3. If the image is colour, what is the correct colour mapping?

4. If the image is gray scale, how many levels are there?

5. What is the correct photo interpretation of the pixel values?

6. What is the scale of the image? (Pixels/cm, pixels/inch)

7. Is the image file compressed and, if so, what type of compression is used?

8 . What is the image's orientation?

Without this information, a bit map of the pixels of an image is a meaningless list of numbers. As such, some means of relaying this and other crucial information must be provided to the machine. This requirement led to the development of a variety of image file formats. As formats evolved, a number of problems were encountered which left the industry striving to find improvements to existing formats (like PCX) or to develop of a new format which could become the industry standard. The Tagged Image File Format was born of this effort and was designed to be a flexible and comprehensive means for storing bi-level, gray scale or full colour digital images. Its success is evidenced by the fact that every major scanner and desktop publishing program supports TIFF [Lind91].

In essence, images stored in TIFF contain a bit map of the pixel values of the image and a group of standardized descriptive tags which provide crucial information on the type of image and how it is to be displayed. The developers of TIFF designed the format to accomplish three goals: extendability, portability and revisability [Lind91]. The first goal was intended to allow for the addition of new image types and fields to the array of descriptive tags as new 
developments arose. Additionally, all older applications were to be able to decipher newer images within the limits with which they were designed. The second goal was intended to ensure that TIFF images could be interpreted regardless of the machine or operating system which was reading them (such as Intel and Motorola systems). The final goal was adopted to ensure that images stored in TIFF could be edited as well as read.

So, how does the TIFF system work? All TIFF files are comprised of three basic components: an image file header (IFH), an unlimited number of image file directories (IFDs) and the image's pixel information (see Figure 2-1). A TIFF header is an eight byte sequential data segment which is always located at the beginning of a TIFF file. It is, in fact, the only TIFF element which has a fixed location. It is made up of three fields which indicate order, version and the offset to the first IFD. The order field is a two byte segment which, at present, is always either " $\Pi$ " or "MM" denoting Intel-style ordering or Motorola-style ordering. Note that this is not meant to imply that an Intel machine cannot read a TIFF file written in Motorola-style ordering, rather that the file has been saved in a fashion that is easiest for the type of operating system specified to read. If this field contains the value $I I$, each segment of bytes from that point onwards is to be read right- to-left, ie a four byte segment containing the hexadecimal values $00 I E 330 A$ would be joined together and read as the single hex value $A 33 I E 00$. If the Motorola -style ordering is used, segments are to be read left-to-right. Thus the value of the segment stated above would be $I E 330 \mathrm{~A}$ (hex). The version field is a two byte segment which is byte-order sensitive and always contains the value 42 if the file being read is a TIFF file. It is not, as one might expect, intended to refer to the version of TIFF that the image was stored in but rather just to confirm that a TIFF reader can read this file. The final field is an four byte 
segment which provides the offset, from the beginning of the file, to the first IFD (note again that this, like all segments, is byte-order sensitive).

An IFD is basically a collection of descriptors (labelled with tags) for the image contained in the file. It is comprised of three fields: count, array and offset. The count field is a two byte segment which indicates the number of 12 byte segments that are contained in the array field. The array field is comprised of count 12 byte segments, each of which is referred to as an IFD entry. The offset field is a four byte segment which indicates the offset, from the start of the file, to the next IFD. Its value is 0000 if no further IFDs exist. If there is more than one IFD present, the file contains more than one image.

IFD entries are used to describe some characteristic of the image and indicate where in the TIFF file the details of the description can be found. The 12 bytes are broken down into four fields: tag, type, count and valofs. The tag field is a two byte field which is a standardized index used to describe the information to which the entry refers. For example, the tag 256 (dec) informs the reader that the entry contains information on the width of the image in pixels. There are a wide variety of tags which, together, cover all the information that a machine would require to interpret the image stored within the TIFF file. As new capabilities in image display and processing emerge, new tags can be added to the library of standardized tags available; thus fulfilling the first goal of the TIFF designers. A list of common tags is attached as Appendix D for easy reference. It should be noted that each TIFF file contains only those tags required to interpret the image contained within the file. The next field is a two byte segment which indicates the type of data to which the entry refers. Appendix D also contains a list of TIFF data types. The count field is a four byte segment which specifies the number of elements of the 
specified type which comprise the tag's information. The size of the tag's data is thus the product of the count field and the size of the type of data referenced:

$$
\text { data_length }=\text { count } * \text { sizeof }(\text { type }) \text {. }
$$

If data_length is less than 32 bits, the four byte valofs field contains the information required. If it is greater than 32 bits, the valofs field is an offset (from the start of the file) to the first memory location where the information is stored. For reasons which are not apparent, if the valofs field contains the tag's data, it is left justified regardless of the order specified in the header [Luse93].

The actual bitmap of the pixels which comprise an IFD's image is stored in one or more strips within the file. Three tags indicate how the bitmap is stored:

273 (hex) - strip offsets: an array of count strip offsets. If there is only one strip (usual case) valofs is the offset to the entire sequential bitmap. If there are multiple strips, valofs points to the first memory location containing the first of count four byte strip offsets;

279 (hex) - strip byte counts: number of bytes within each strip; and

278 (hex) - rows per strip: number of image rows covered by each strip. Rows = height if only one strip is used.

Strips are used in order to allow IFDs and IFD entry data to be stored anywhere within the TIFF file. Usually only one IFD is present, stored at the end of the file, and all of the pixel information is contained in one strip between the IFH and the IFD. However, the ability to break the image down into multiple strips, use multiple IFDs and store all information except the IFH anywhere within the TIFF file is provided for in order to allow the format to be as flexible as possible. Figure 2.1 provides a graphical illustrations of the IFH, IFD, IFD entry and a standard TIFF file layout. 


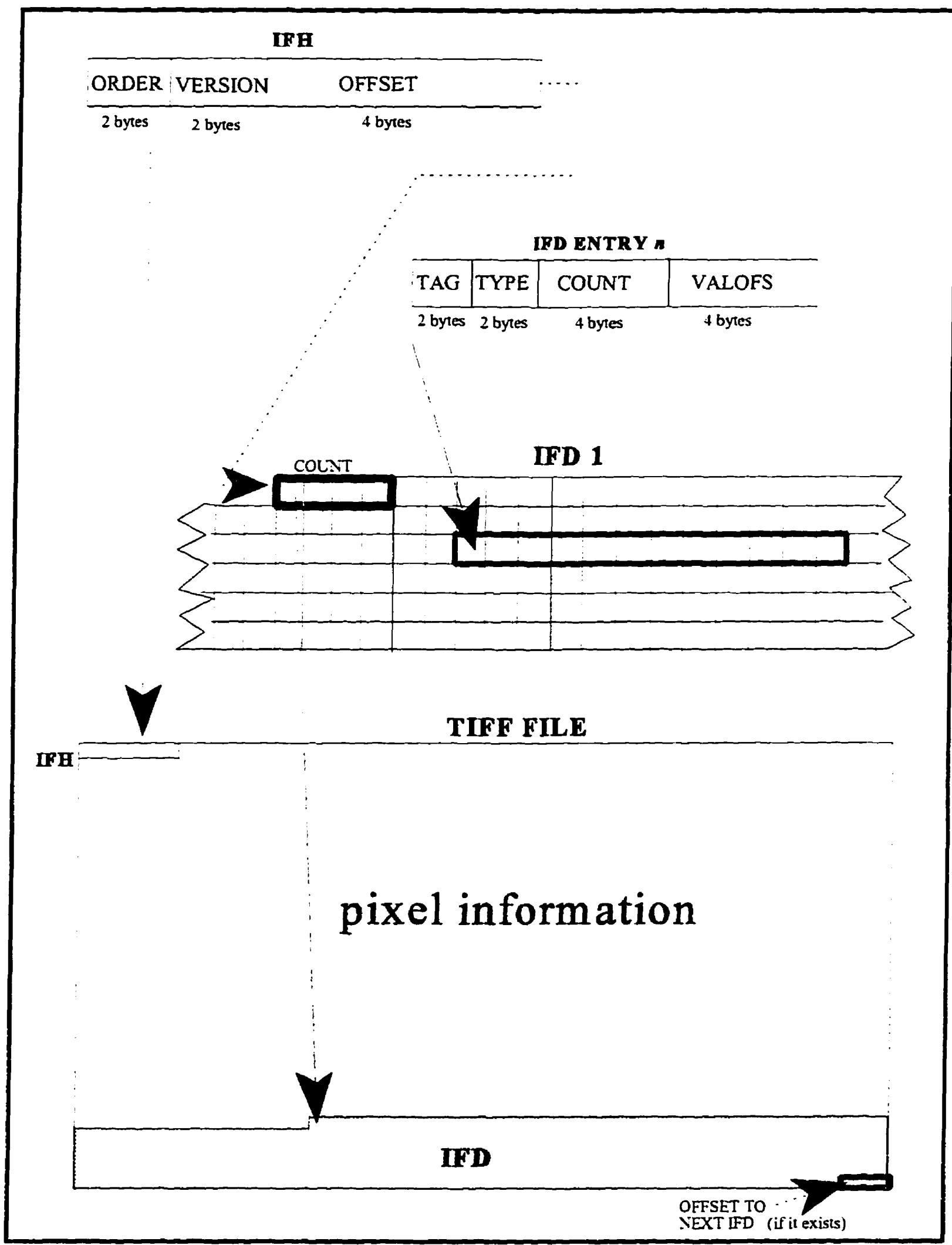

Figure 2-1 IFH, IFD, IFD Entry and Standard TIFF File Layout 
The final aspect of TIFF files that merits specific mention is file compression. As was pointed out previously, a vast amount of storage space may be required to store the bit map of the image. To counter this, the specifications for TIFF recommend the support of four different types of file compression. The tag 259 (103 hex) is used to label the IFD entry which indicates the type of compression that is used within the TIFF file. Currently, there are six standard values (ValofS) for compression formats which may be supported [Lind91]:
( ValorS $=1$ )
( ValofS $=2$ )
No compression but bytes are tightly packed;
$($ ValofS $=3$ )
CCITT Group3 1-Dimensional modified Huffman RLE:
( ValofS $=4$ )
Facsimile compatible CCITT Group 3;
( ValofS $=5$ )
Facsimile compatible CCITT Group 4;
$($ ValofS $=32773)$
LZW (Lempel-Ziv \& Welch); and
Packbits (MacIntosh).

All TIFF readers must support at least one of these formats. In any case, it must be emphasized that only the raster information of a file is compressed under any of these schemes: the IFH, IFDs and IFD entries are never compressed. For this research, only ValofS $=1$ is supported, ie compression techniques were not investigated. For more information on the compression schemes outlined above, the reader should refer to Lindley's (1991) text.

\section{2-3 M-mode Echocardiograms}

This paper is not concerned with the specific analysis of images used in Echocardiography. Such matters are left to highly skilled professionals who are well versed in the human anatomy and trained to decipher the information contained in such images. Rather, the material presented below is aimed at providing background to the reader on the ultrasound process so that the benefit of techniques for enhancing images described in later chapters can be 
readily associated with the "improvement" of images such as these. In the case of Echocardiography, improvement would depend on the reduction of noise and sharpening of lines within an ultrasound image without a loss of spatial precision. So, before using an M-mode image as the subject of examples of edge detection and enhancement, we must first look at what these images represent.

Initially, the primary means used to detect heart problems in patients was angiography which uses X-ray technology. With the development of ultrasound, doctors were provided with a means of diagnosing patients without the use of potentially harmful ionizing radiation techniques [Pell93]. Ultrasound uses the same technology as that used to detect submarines (asdic/sonar) and was thus heavily influenced by military scientific advances during the Second World War [Laur81]. Due to the absence of side effects, since that time ultrasound has become one of the most widely used techniques for evaluating cardiac performance and detecting/diagnosing cardiac problems in patients [Pell93] [Ruiz95] [Unse89].

Ultrasound imaging uses the reflection of high frequency sound to observe internal organs within patients [Tuai85]. The ultrasound waves are normally produced by exciting a piezoelectric element with a rapidly varying voltage [Laur81]. This acoustic energy, typically having a frequency of approximately 3 to 7 megahertz, travels through the body as a longitudinal pressure wave front [Tuai85]. If the wave hits a region with a higher or lower density the wave will be partially reflected back to the element where it is converted into an electrical signal whose amplitude is proportional to the strength of the returning wavefront. Higher frequency waves are better at detecting detail within the subject structure (hence higher resolution): however, the higher the frequency the lesser the penetration as higher frequency waves lose their 
energy detecting these smaller changes and thus have less penetrating power. As such. ultrasound instruments can be set at a higher frequency to detect structures in the near field (closer to the element and influenced by diffraction) and lower frequency to detect changes in the far field (further from the element and not subject to diffraction) [Tuai85]. Thetime that it takes the wave to return provides information on the distance to the structure causing the reflection and the strength of the returning wavefront indicates the relative difference in acoustical impedance that was encountered.

There are several different ways in which ultrasound information can be displayed. These include [Laur81]:

A-mode: Also called amplitude mode, this format displays the amplitude of reflected echos as vertical spikes on a horizontal axis calibrated for distance (from the element);

B-mode: $\quad$ Same principle as A-mode except that echos are shown as dots whose intensity reflects the amplitude of the reflected energy. Also called twodimensional imaging [Tuai85], this technique provides cross-sections of slow moving or stationary structures; and

M-mode: A continuous flow of B-mode images along one dimension recorded over a period of time.

For the results chapter of this paper, we are concerned with M-mode Echocardiography. A-mode was not considered as it is seldom used except for calibration and it provides much less information than either B-mode or M-mode. B-mode is a suitable candidate; however, it is 
relatively inaccurate for measurements such as wall thickness and M-mode is widely accepted in this regard [Pel193] [Coll87]. As Dr Shoucri is concentrating on M-mode for this reason, an M-mode image was thus selected. The processes described in the following chapters can. nonetheless, be applied to any of these techniques.

A typical M-mode image consists of a set of wavy lines moving left to right across the image. The horizontal axis relays the time that echos are received, starting on the left. The vertical axis relates spatial information to the user - the distance that the echo occurred from the element. The location of the element is at the top of the axis. The brightness of any pixel on the image indicates the relative strength of the echo received.

Unfortunately, images obtained through the use of ultrasound are often degraded by "speckle." [Tuai85] [Ruiz95] Speckle is the result of the phase sensitivity of the ultrasound process; reflected waves can have their amplitudes altered by constructive and destructive interference from other returning wavefronts. These other wavefronts typically result from the scattering of beams by structures encountered whose lateral dimensions are less than or equal to the wavelength of the beam [Tuai85]. It is interference such as this and the noise generated by the sensors which are used in Echocardiography [Pell93] [Unse89] which undermine the benefits and degrade the accuracy of the analysis of ultrasound images.

As the wavefronts penetrate a heart under examination, interfaces between the cardiac wall, valves, blood and muscle tissue cause echos which are detected by the element. The flow of blood, stroke volumes, thickness of walls, and other crucial parameters can also be deduced from the images [Unse89]. Such data is determined by analysts who manually take measurements between structures contained within the M-mode images. Consequently, it is 
crucial that the information in an M-mode image be as clear and sharp as possible. Any reduction in noise and speckle would benefit measurement as would a sharpening of the exact location of edges within the image - leading to a higher accuracy in measurements and an increased chance of disease detection [Tuai85] [Unse89]. Throughout this paper, numerous means by which this enhancement may be accomplished will be discussed and examples of the results of some of the techniques when applied to an M-mode image will be provided.

\section{2-4 Image Processing}

\section{2-4.1 General}

Now that some of the peripheral background theory behind the thesis has been presented. the next stage is to begin an exploration of operations that are commonly done to digital images. The types of operations that are applied to an image are application specific. For this work. a number of operations have been selected which can be applied during the analysis of M-mode Echocardiograms - which have the added benefit of being reasonably common in other image processing applications.

To begin with, it is necessary to briefly review some of the concepts associated with computer vision. As computers work with numerical rather than pictorial or analog 
information, it is necessary to sample the continuous domain (the real world). This sampling process is called digitization and involves the division of continuous images into small equal sized parts called picture elements or pixels [Cast79]. These elements generally form an array of $\mathrm{N}$ columns and $\mathrm{M}$ rows (see figure 2-2). A pixel at spatial coordinates $(x, y)$ is represented by an integer value which represents the sampled intensity of the original image at that location. The entire digitized image can then be represented mathematically as a discrete function:

$$
\begin{gathered}
f(x, y) \quad \text { where } x, v \in \text { Integer } \\
0 \leq x<M \text { and } \\
0 \leq y<N
\end{gathered}
$$

For this thesis, the integer value for each pixel represents a gray value for the intensity at that point. The number of gray levels that are used depends on the hardware limitations of the digitizer and the preference of the user. In this case, 256 gray levels is the established norm. As such, each pixel requires one byte of storage space to store its intensity value - eight bits allows the storage of numbers between $0\left(2^{0}-1\right)$ and $255\left(2^{8}-1\right)$.

Algorithms used in the processing of these digitized images fall into four basic categories: point, area, algebraic and geometric [Cast79] [Bank92]. Point processes are all operations wherein pixels are changed based on a mathematical operation on that pixel's value only. The value of neighbouring pixels or the pixels of other images are not involved in these processes. They are used to provide contrast enhancement, contrast stretching and gray scale manipulation [Cast79]. An example of a point process is negation where, in our case, a pixel's gray value is inverted by subtracting its original value from 255; ie white (0) becomes black (255) and vice versa. 


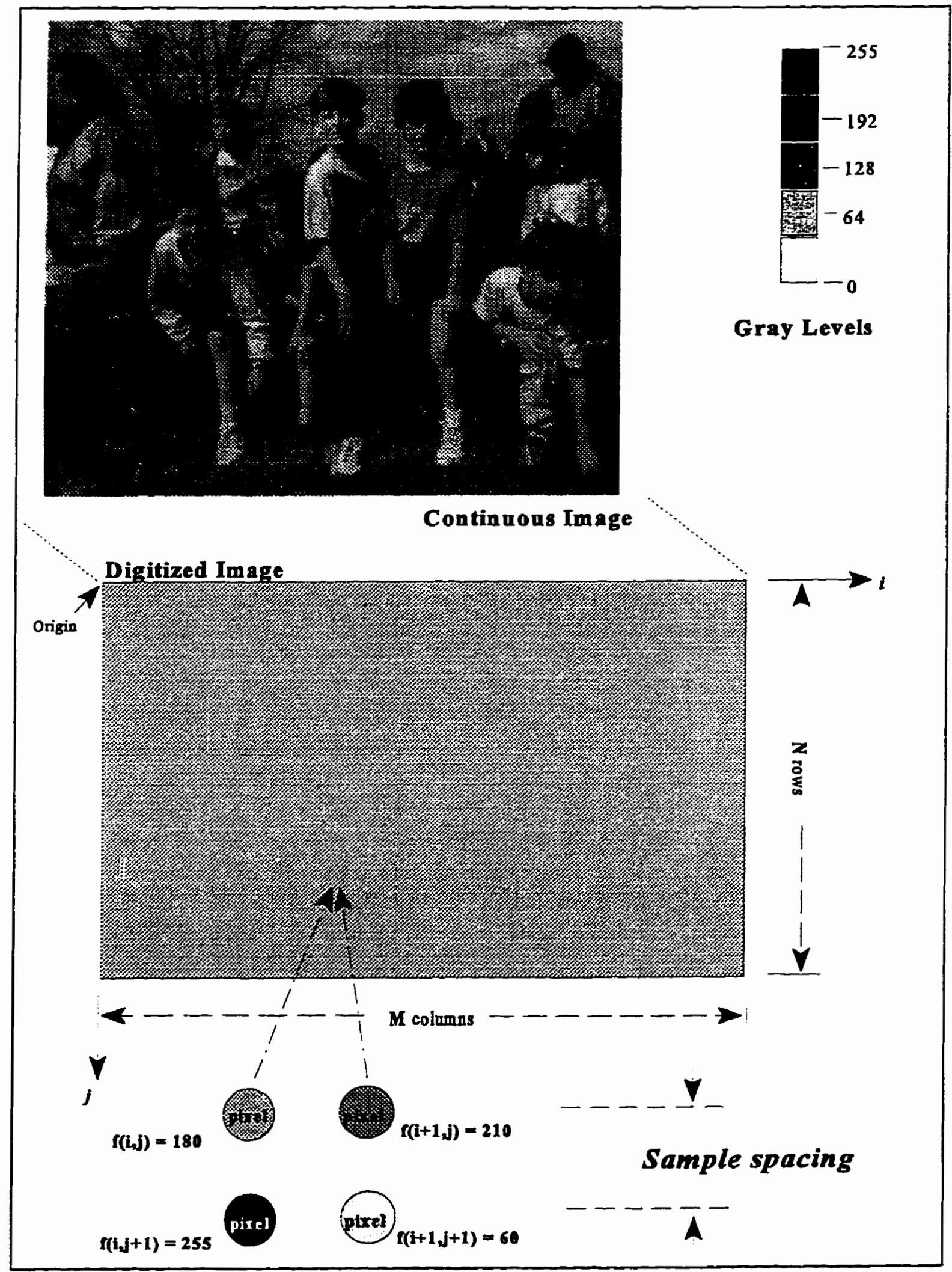

Figure 2-2 The Digitization of an Image 
Area processes are those processes where each pixel is altered by some mathematical operation done on it and a number of its neighbours. The number of neighbours involved depends on the application. These processes generally involve a procedure called convolution which will be discussed in more detail shortly. Some examples of area processes include smoothing, differentiation, and Laplacian filtering.

The next type of process, algebraic, involves operations which require two images in order to arrive at the end result. In this case, some mathematical operation is done on a pixel-bypixel basis for pixels at the same spatial coordinates within the two images. These processes are particularly useful in the reconstruction of an image from multiple images of the same scene which have each been degraded in some fashion. Other examples include the simple addition. subtraction, multiplication and division of images.

The final type of process is geometric. In geometric processes, the value of a pixel is not altered, rather its spatial coordinates are changed. Some examples of these processes are scaling, flipping and rotation, where each pixel within an image is moved from some position $(\mathrm{x}, \mathrm{y})$ to a new location $\left(x^{\prime}, y^{\prime}\right)$ according to an appropriate mathematical relation.

Now that we have considered the types of processes, we can now explore a number of specific operations which can be performed on digitized images. These operations have been grouped according to whether they are unary or binary in nature. Unary operations include all operations which involve only one image. Conversely, binary operations are operations which involve two images. It should be noted that operations done on a large number of images can always be broken down into single stages involving either a unary or binary operation. As such, 
all operations fall into these two categories, and each operation involves one of the four types of processes described earlier.

\section{2-4.2 Unary Operations}

Initially, there were seven types of fundamental unary operations that were studied as part of this research. They include negation, brightness adjustment, cropping, scaling, histogram equalization, histogram thresholding and median filtering. Chapter four will provides the theoretical background for two additional unary operations related to edge detection.

The first operation to be considered is a point process and is the simplest operation negation. To negate an image, the following transformation is applied:

$$
f_{n}(x, v)=255-f(x, v)
$$

The result of this transformation is to make all black pixels white, make all dark grey pixels light grey, have all mid-gray pixels remain mid-gray, make all white pixels black and so on. The end result is directly analogous to photographic negatives.

The next operation, again a point process and also one of the simplest, is brightmess adjustment. As the name implies, this operation aims at improving an image's detail by adjusting the brightness of its pixels. There are three ways in which this can be done. The first is by the simple linear addition or subtraction of a constant value from each of the pixels. This uniformly brightens or darkens the image. Mathematically, this can be represented as follows:

$$
\begin{aligned}
& f_{B C}(x, v)=f(x, y) \pm \Delta \\
& \text { where } \Delta \in \text { Integer }
\end{aligned}
$$


The second is by adjusting each pixel value to some percentage of its original value (non-linear):

$$
\begin{aligned}
& f_{B P}(x, y)=f(x, y) \times \Delta \\
& \text { where } \Delta \in \Re \text { and } 0 \leq \Delta<\infty
\end{aligned}
$$

The final type is logarithmic. In this case, the image function has each pixel value adjusted to its logarithmic value:

$$
f_{B L}(x, y)=\log [f(x, v)]
$$

In the latter two cases, different intensities within the image are emphasized. In the first case. the entire image is brightened or darkened uniformly. In the second and third cases, the transform increases or decreases the overall brightness of the image and can be used to improve the contrast in the darker regions. One question which arises out of the application of these operators is what to do about pixels whose values are adjusted outside of the range $0 \rightarrow 255$ ? Generally, the simple solution is followed and those values over 255 are set to 255 and those below 0 are set to 0 . Another possibility is to normalize the entire distribution of intensities such that the pixel(s) with the highest intensity value are set to 255 , the lowest is (are) set to 0 and the ones in between are scaled between 0 and 255. In this work, the former procedure is used.

Cropping is the first example of a unary operation which is geometric. It involves the removal or cropping of one section of an image. Mathematically, this can be represented as:

$$
\begin{aligned}
& \text { image } \\
& f(x, y): \begin{array}{l}
0 \leq x<M \\
0 \leq y<N
\end{array} \Rightarrow f_{C}(x, y) \quad \text { cropped image } \\
& \text { where } f_{C}(x, y)=f(x, y) \text { for } \begin{array}{l}
x_{\min } \leq x \leq x_{\max } \\
y_{\min } \leq y \leq y_{\max }
\end{array} \\
& \text { and } f_{C}(x, y)=0 \quad \text { otherwise }
\end{aligned}
$$


Here $\left(\mathrm{x}_{\min }, \mathrm{y}_{\min }\right)$ is the upper left comer of the area to be cropped and $\left(\mathrm{x}_{\max }, \mathrm{y}_{\max }\right)$ is the lower right comer of the area to be cropped. Note that the value of any pixel is not altered, only the spatial coordinates and limits of the image function are changed.

Another unary operation which is geometric is scaling. If an image is made larger, a number of pixels will end up being duplicated in order to "fill-in" the larger image. Conversely. if an image is reduced in size, some pixels are lost. The algorithm for scaling an image is as follows:

1. Determine the scaling factor, $\delta$

2. Multiply the width and the height of the old image by $\delta$ to get the new images width and height

3. Set a vertical counter, vcount (real) to 0

4. For each row of the new image

- set a horizontal counter, xcount (real) to 0

- for each column of the new image

- copy the pixel in the old image at Integer(xcount), Integer(vcount) to the new image

- increment xcount by $\delta$

- increment ycount by $\delta$

As the scaling factor is increased, larger and larger squares will be displayed to the point that they are readily visible within the image. This occurs because there is no allowance made for interpolating values for the new pixels located in between the original ones. As they are merely taking on the value of their closest neighbour both vertically and horizontally, squares are formed. Conversely, as images are reduced, there is a loss of information within the image which cannot be recovered without referring to the original image.

The next two processes, thresholding and histogram equalization, are point processes and they are based on histogram modification. A histogram of an image is "a graphical representation of the frequency count of the occurrence of each intensity in the image."[Galb90] 
In our case, the number of possible gray values is 256 , labelled $0-255$ along the abscissa. The vertical axis of the histogram is linearly scaled from $0 \rightarrow$ maxcount where maxcount is the greatest frequency count for any gray level within the image. The histogram of an image thus contains no spatial information whatsoever.

Once the histogram of an image is obtained, a user can implement an operation called thresholding. Thresholding is the altering of all pixel values below a lower limit ' $l$ ' to 0 and/or the altering of all pixel values above an upper threshold ' $u$ ' to 255. For example, if a user opted to threshold an image at $l=20$ and $u=200$, all pixels with an intensity of $0-20$ would be changed to 0 and all pixels with an intensity of $200-255$ would be set to 255 . This technique is useful in emphasizing certain intensities within an image by increasing their contrast with the background or removing them by making them the same intensity as the background.

A histogram has other useful properties. A narrow histogram, for example, indicates a low amount of contrast within an image [Galb90]. Hence, by spreading out the distribution of intensities, we improve the contrast within the image. Recall that it is easier for the human eye to see contrast within an image if neighbouring pixels have large differences in their intensity values. The farther apart the gray levels, the easier it is to notice the change. One means of creating this effect is through histogram equalization. An equalized histogram is a histogram whose gray levels have a uniform density. As such, intensities which occur more frequently are spread out more than those used less frequently. To create this new histogram, we begin with the histogram information of the original image and denote the frequency count at any intensity ' $k$ ' for the original image ' $A$ ' as $N_{A}(k)$. We next look at the cumulative histogram of ' $A$ ' which is a graphical representation showing, for each gray level ' $k$ ', the total number of pixels within 
the image whose gray value is $\leq \mathrm{k}$. Mathematically, this is expressed as

$$
C_{A}(k)=\sum_{j=0}^{k} N_{A}(j) \quad 0 \leq k \leq k_{\max }
$$

where $k_{\max }$ is the highest gray value possible (in our case 255 ). We then calculate the probability of a pixel having a value $\leq \mathrm{k}$ as

$$
F_{A}(k)=\frac{C_{A}(k)}{n_{A}}
$$

where $\mathrm{n}_{\mathrm{A}}$ is the total number of pixels in ' $\mathrm{A}$ '. We have then that

$$
\begin{gathered}
0 \leq F_{A}(k) \leq 1 \\
\text { and } F_{A}\left(k_{\max }\right)=1
\end{gathered}
$$

We use these formulae to calculate the equalized histogram gray value frequency counts, $N_{B}(k)$ as follows

$$
N_{B}(k)=k_{\max } \cdot F_{A}(k)
$$

$N_{B}(k)$ is the equalized histogram values of ' $A$ ' and this histogram could then be displayed in the same manner as the original histogram values $N_{A}(k)$. The new image, ' $B$ ' is created from these adjusted gray values. 
The next area of discussion for this chapter, and the subject of the next, is filtering. Filters are an area process which manipulate the pixel values of an image by looking at the neighbours of the pixel. Linear filters do so through a process called convolution. Although median filtering is a non-linear technique and does not involve convolution, it effects its area process through the use of a filter mask - of which a convolutional mask is a subset. Thus, before proceeding with a discussion of filters it is important to understand the concept of convolution and filter masks.

Convolution comes from linear systems theory which is a branch of mathematics used to describe the effects of various filters on electrical and optical systems [Nibl86]. In the continuous sense, convolution in one dimension is expressed as

$$
g(x)=\int_{t=-\infty}^{\infty} f(t) h(x-t) d t
$$

where $h(t)$ is called the impulse response and completely characterizes the filter [Nibl86], $g(x)$ is the output of the filter and $f(x)$ is the input. In the discrete case, this becomes

$$
g(i)=\sum_{k=i-w}^{i+w} f(k) h(i-k)
$$

where the impulse response function is 0 outside of a finite range $-\mathrm{w} \leq k \leq \mathrm{w}$. This means that the output $\mathrm{g}(i)$ for any point $i$ is a weighted sum of input pixels surrounding $i$ 
(within $\pm w$ pixels) where the weights are given by $h(k)$. In two dimensions. we have

$$
g(i, j)=\sum_{k=i-w}^{i+w} \sum_{l=j-v}^{j+v} f(k, l) h(i-k, j-l)
$$

where the filter is of dimension $2 w+1$ by $2 v+1$.

Effectively, discrete convolution is effected by "sliding" the mask, $\mathrm{h}$, over the original image, $f$, from left to right starting from the top and, for each pixel, summing the weighted (using the coefficients of h) $2 w+1$ by $2 v+1$ neighbouring pixels for each point . Note that the output of the filter operation will be an image which is $2 w$ pixels narrower and $2 \nu$ pixels shorter than the original. This occurs because the mask begins $w$ pixels in and $v$ pixels down from the top left corner of the image. All border pixels are normally lost because there is not sufficient image information available to apply the mask at these points. Extrapolation procedures can be involved such that the output is the same size as the original image: however, in this thesis all border regions are simply lost.

Convolution has several very useful properties which will be used in mathematical derivations in the next chapter. Specifically, convolution is:

1. communicative

2. distributive over addition

3. associative

$$
\begin{array}{ll} 
& f^{*} g=g * f \\
& f^{*}(g+h)=f^{*} g+f^{*} h \\
\text { or } \quad & \frac{d}{d}\left[g^{*} h\right)=\left(f^{*} g\right) * h \\
& d t g]=f^{\prime} * g=f * g^{\prime}
\end{array}
$$

for both digitized and continuous domains. 
Median filtering is a non-linear technique designed to smooth an image and reduce the effects of speckle, or isolated spike, noise. An MxN median filter mask (where $\mathrm{M}$ and $\mathrm{N}$ are odd) takes the $\mathrm{MxN}-1$ neighbours of a point $(i, j)$ and the point itself and orders the pixel values from lowest to highest. The median value is then determined, i.e. the pixel value where $50 \%$ of the other pixel values are below it and $50 \%$ are above it. This number is then recorded for that point. This processes continues for all non-border points in the image (as discussed above). The resulting image will have speckle noise removed as any singular speckles are removed during this operation for they can never be the median value when surrounded by pixels which are not "noisy." The drawback to median filtering is that it can mottle an image by smoothing over texture and edge information [Tuai85].

\section{2-4.2 Binary Operations}

By definition, all binary processes are algebraic processes as they always involve two images. There are never more than two images involved in any one process because, as mentioned earlier, all image processes can be broken down into a series of unary and binary operations. The binary operations that will be discussed as part of this work are addition, subtraction and multiplication. The division of two images is similar in nature but is not covered here as no useful application within the spatial domain could be discovered.

The first process, addition, involves the pixel-by-pixel addition of two images to produce a third image. Mathematically, this is expressed as:

$$
\begin{array}{r}
A(i, j)=f(i, j)+g(i, j) \quad \text { for } 0 \leq i<M \\
0 \leq j<N
\end{array}
$$


where $A(i, j)$ is the output image and $f(i, j)$ and $g(i, j)$ are input images of equal dimension $(M x N)$. An important application of this technique is the averaging together of degraded images of the same scene to reduce the effects of random noise. Addition may also be used to superimpose one image upon another. A problem is encountered in that some pixels may end up with values $>255$. To counter this, a person can either set all output $>255$ at 255 or normalize the values resulting from the addition. That is, determine the lowest result, $l$, the highest result, $h$, and transform all intermediate values using the formula

$$
\text { newvalue }=255 \cdot \frac{\text { oldvalue }-l}{h-l}
$$

The next binary operation is subtraction. Mathematically, this is expressed as:

$$
\begin{array}{r}
A(i, j)=f(i, j)-g(i, j) \text { for } 0 \leq i<M \\
0 \leq j<N
\end{array}
$$

where $A(i, j)$ is the output image and $f(i, j)$ and $g(i, j)$ are input images of equal dimension $(M x N)$. Again, the problem arises as to what to do with values falling below 0 . As with addition, the output image can simply set all pixels with values $<0$ to 0 or normalize the result using the same equation as shown above. Subtraction is typically used to remove an undesired pattern or section from an image. This may be some noise or other contamination within an image. It can also be used to detect the differences between two images of a scene. This is particularly useful in motion analysis and in some edge detection schemes (see Chapter Three).

Multiplication is the last binary operation to be covered. It is used primarily in the frequency domain (low and high pass filtering) although it can be useful in the spatial domain 
when one wishes to "mask-off" a certain portion of an image. For example, a doctor may wish to edge enhance a small section of a large MRI image in order to save time during what could be a rather exhaustive computation, then add that section's contour map back to the original image. To do this, he/she would blank-out a large portion of the original image by multiplying it with a mask which removed all detail outside of the required area. When the enhancement algorithm is applied to this smaller image, much fewer computations could be required if the process was of $\mathrm{O}(n \log n)$ or $\mathrm{O}\left(n^{2}\right)$ for example. The processed image (still the same overall dimensions as the original) could then be easily added to or subtracted from the original. Of course, if a capability to crop and overlap is available, this same process could be accomplished in that manner instead.

The multiplication operation is expressed mathematically as:

$$
\begin{array}{r}
M(i, j)=f(i, j) \cdot g(i, j) \quad \text { for } 0 \leq i<M \\
0 \leq j<N \\
\text { and } i, j \in \text { Integer }
\end{array}
$$

where $M(i, j)$ is the output image and $f(i, j)$ and $g(i, j)$ are input images of equal dimension $(M x N)$.

Having examined the types of processes that can be performed on images and several fundamental operations, the next chapter will explore the more intricate problem of detecting edges, or contours, within digitized images. 


\section{Chapter Three}

\section{Edge Detection - Theory}

\section{3-1 Introductory Comments}

One of the most useful operations in automated image analysis is edge detection [Torr86][Chen89]. The extraction of features such as edges is essential in the classification of boundaries, matching images, and texture analysis and can be further developed into routines for target recognition, character recognition, scene analysis, motion analysis and numerous biomedical and industrial applications [Ekst84] [Torr86] [Cann86] [Free91][Deri93]. Most of these applications involve two stages. The first stage is generally the detection and localization of edges. During this stage the analysis of images is simplified immensely as the amount of data to be processed and the effects of noise and irrelevant details are drastically reduced, with a concurrent preservation of useful structural information about object boundaries and their locations [Cann86] [Berg87]. The second stage includes operations such as boundary detection, classification and segmentation [Torr86]. Korn refers to this first stage as low level vision and the second stage as high level vision [Korn88]. The research presented throughout the rest of this chapter addresses only low level vision.

The chapter begins with an outline of the properties of edges. This is followed by an 
overview of many of the mathematical techniques currently employed to detect edges. Two classic approaches are then presented in detail, specifically the Laplacian of a Gaussian (LoG) Filter and the Derivative of a Gaussian (DoG) Filter.

\section{3-2 Properties of Edges}

In general terms, edges are discontinuities in the intensities which comprise an image. These are recorded when a flux of photons or other form of electromagnetic radiation is converted to a set of intensity values by a sensor. They arise from certain physical phenomena including differences in surface reflectance and texture, illumination, surface orientation and depth [Berg87][Marr82]. If sufficient noise is present, it may have the effect of creating false contours or it may degrade/blur existing contours. The ease with which the human eye detects any of these discontinuities as edges within digitized images depends on the relative differences in gray levels between adjoining pixels, ie through relative differences in brightness.

The most basic type of edge occurs when their is a "large" difference in intensity between two adjoining monochromatic areas. "Large" means readily detected by the human eye. For

example, as the human eye can readily detect 40 different shades of gray [Cast79], a difference of one shade is sufficient to be noticeable. Three or more shades is thus more pronounced and so on. This type of edge is called a step edge and examples are provided in Figure $3-1$. In the real world, however, edges are seldom this sharply defined. Normally, they are transitional in 


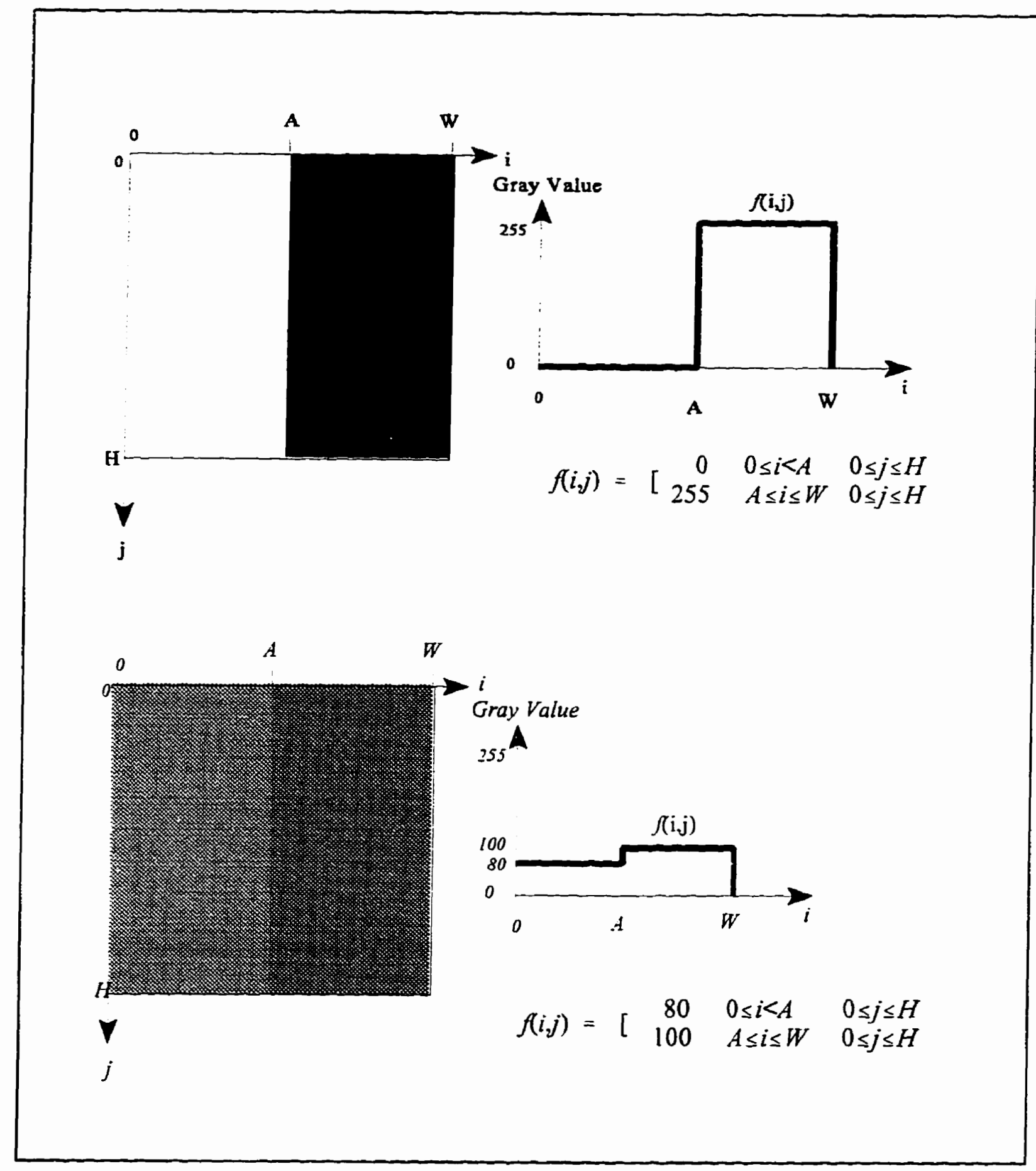

Figure 3-1 Examples of Step Images

nature as opposed to step-like. Further, they are usually degraded by noise to some degree. 


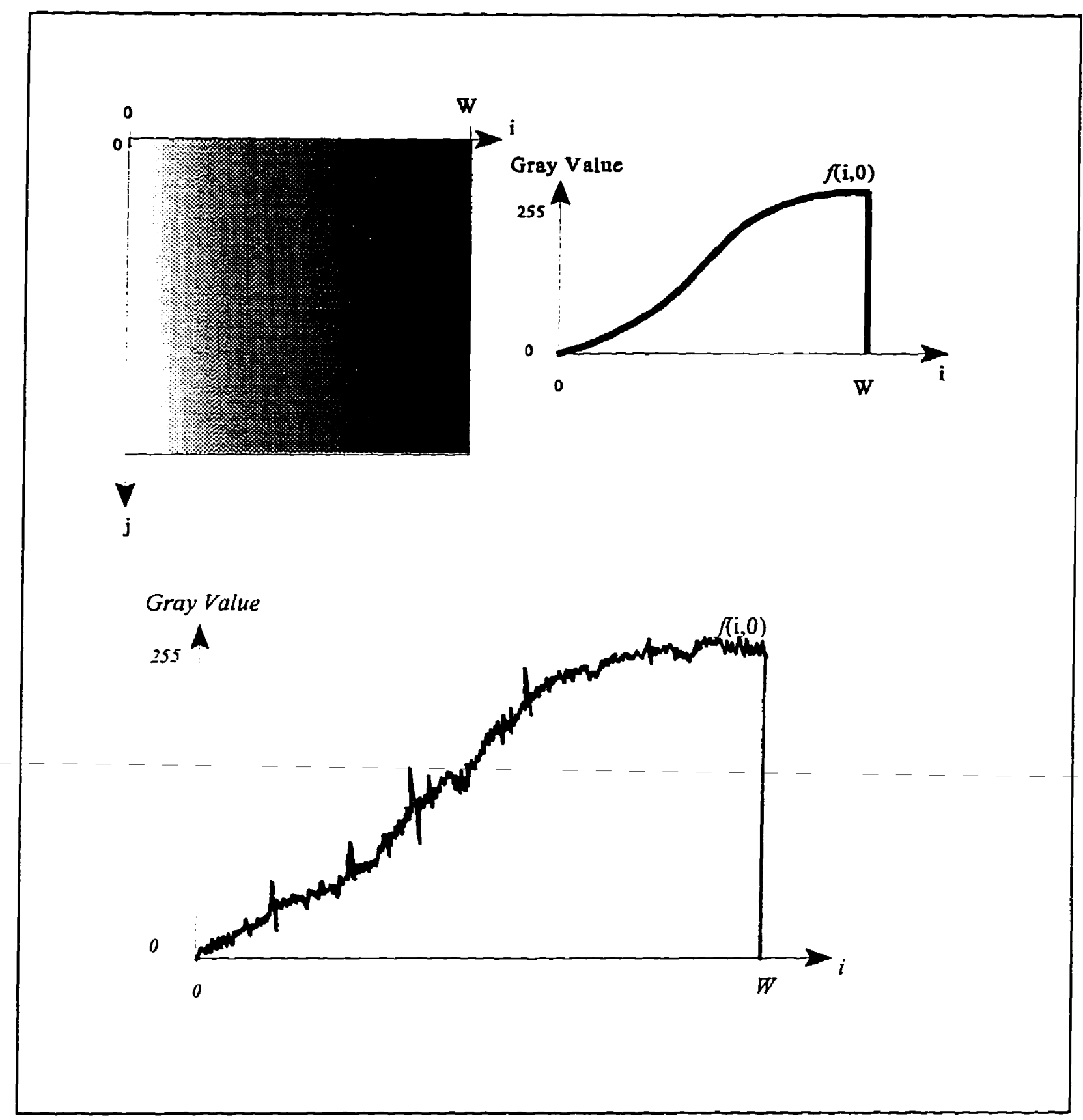

Figure 3-2 Example of Transitional Edge and Noise Effects

Examples of these are provided in figure 3-2. Within an image there will generally be a wide variety of transitional edges composed of different levels for peaks and valleys, differing transitional widths and differing degrees of noise. Additionally, edges may join together or cross 
at $\mathrm{X}, \mathrm{T}$, and $\mathrm{Y}$-type junctions. One classic problem that occurs in the definition of an edge is how does one deal with an edge like that depicted in figure 3-3? Is this two edges at A and B or one edge at point $C$ ? This is a consideration that a researcher or programmer would have to consider based on the requirements of the specific application.

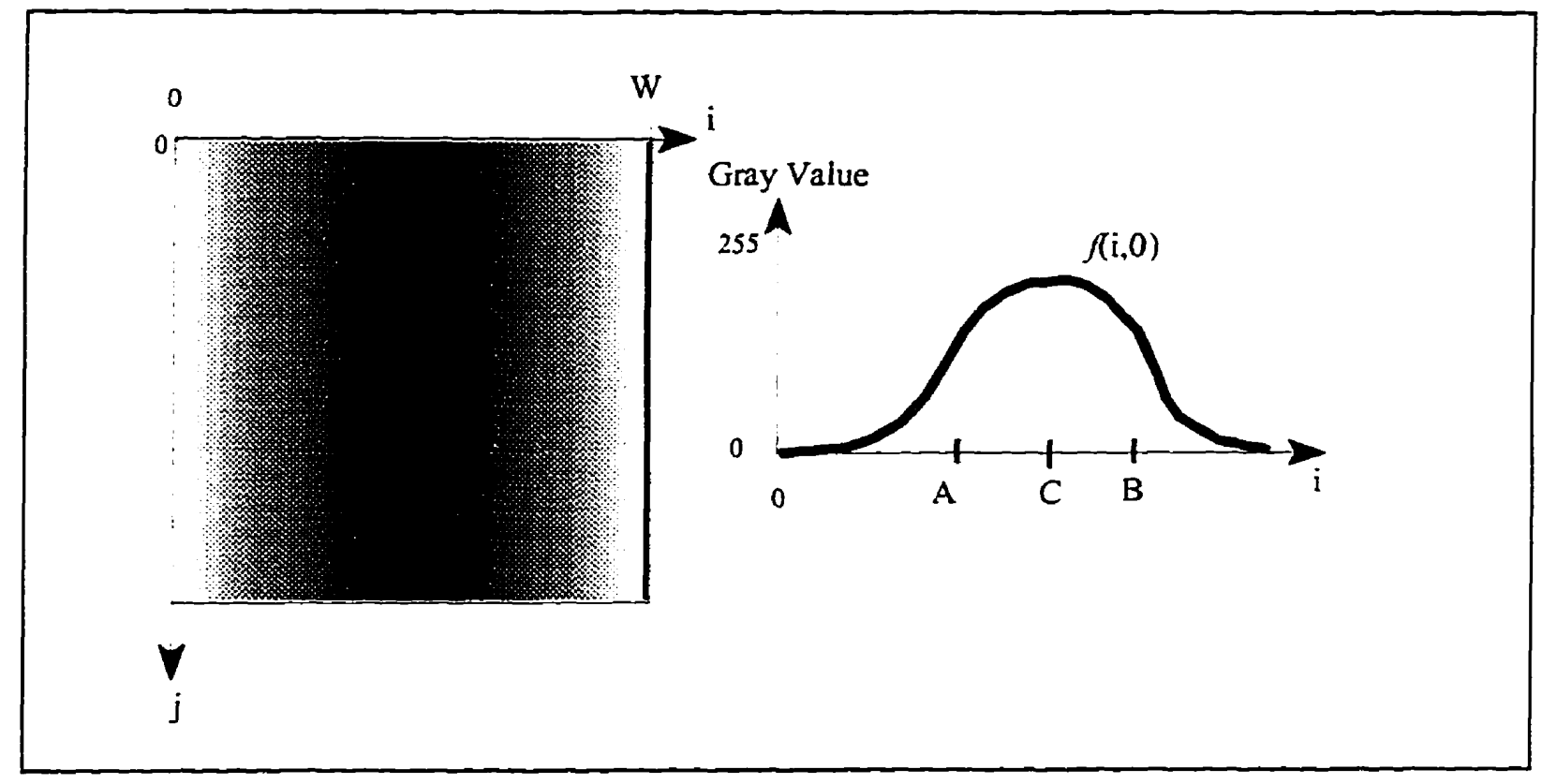

Figure 3-3 Edge Classification Problem

\section{3-3 Overview of Edge Detection Techniques}

As an edge is defined as a noticeable difference in intensities between two neighbouring regions, it stands to reason that the approach one would use to detect them involves the measurement of such changes. Going further, it is clear from this definition that the strength of an edge, ie how easily it is noticed, is dependant on the magnitude of the rate at which the pixel intensities have increased or decreased. In fact, the most common edge detection techniques are 
based on the application of a difference operator which measure rates of change [Nibl86].

Operators of this type are high pass filters. This is evident again from the definition of an edge - around an edge pixel intensities change rapidly and a filter designed to detect them needs to respond to this high frequency of change. One drawback of these filters is that they tend to accentuate noise within the image. Whereas low pass filters attenuate noise through a process akin to integration, high pass filtering is similar to differentiation and, as such, is very sensitive to large changes in intensity [Gonz79]. Consider, for example, an image which is primarily monochrome and dark in nature degraded by a small degree of white shot noise. A smoothing filter (low pass) will average out isolated peaks in intensity caused by the noise leaving a more uniform image. On the other hand, a high pass filter will remove the monochromatic information within the image and respond strongly to the large change in intensity caused by the each noisy pixel. This is caused by the fact that the noisy pixel's intensity is dramatically higher than its neighbours, and edge detection filters are designed to detect and enhance rapid changes in intensity. Figure 3-4 illustrates these effects. It is interesting to note that edges can be enhanced within an image by subtracting a smoothed image from the original image [Neyc93]. This has the effect of creating a relative increase in high-frequency informational content without an increase in noise. An example of this effect is provided in figure 3-5.

There are a number of techniques which have been developed to create difference operators used to detect edges. Most of these can be broken down into two primary groups those that use the first derivative of the image to extract edge information and those that use the second derivative. Examples of each of these are provided below, followed by a detailed discussion on one technique from each category in sections $3-4$ and 3-5. In each case, the 

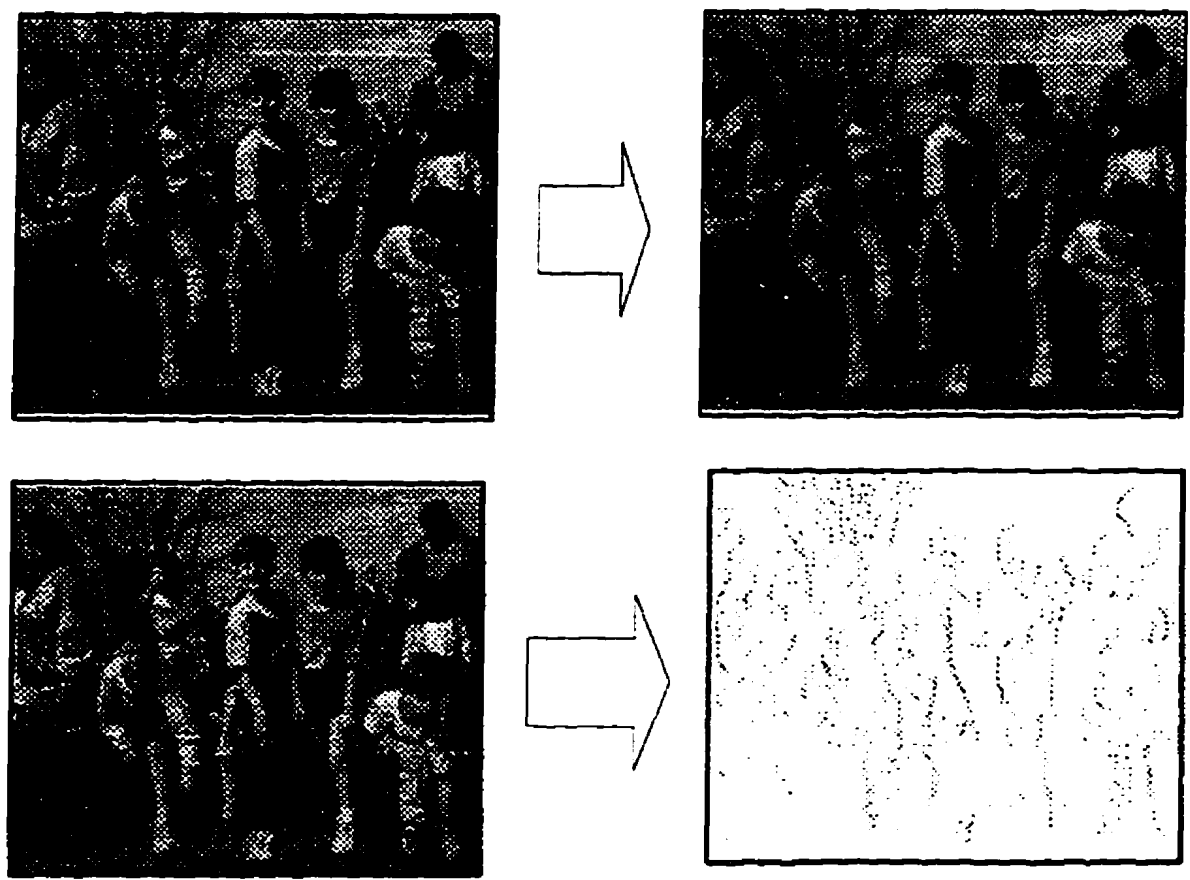

Figure 3-4 Effects of Low Pass and High Pass Filters

brightness changes in a pixel's neighbourhood are used to find the edges within the image [Lind91].

In the ideal, an image with no noise can be edge enhanced by using first derivative operators. For each non-boundary pixel, one can determine the derivative (slope) of the function in any one of eight directions (North, Northeast, East, Southeast, South, Southwest, West, Northwest) using the difference between the subject pixel and its appropriate neighbour [Lind91]. Convolutional masks can be made to determine any one of these eight derivatives. This technique will optimally detect edges aligned in a direction perpendicular to that with which the derivative is taken. Furthermore, these filters will not detect edges aligned parallel to the 


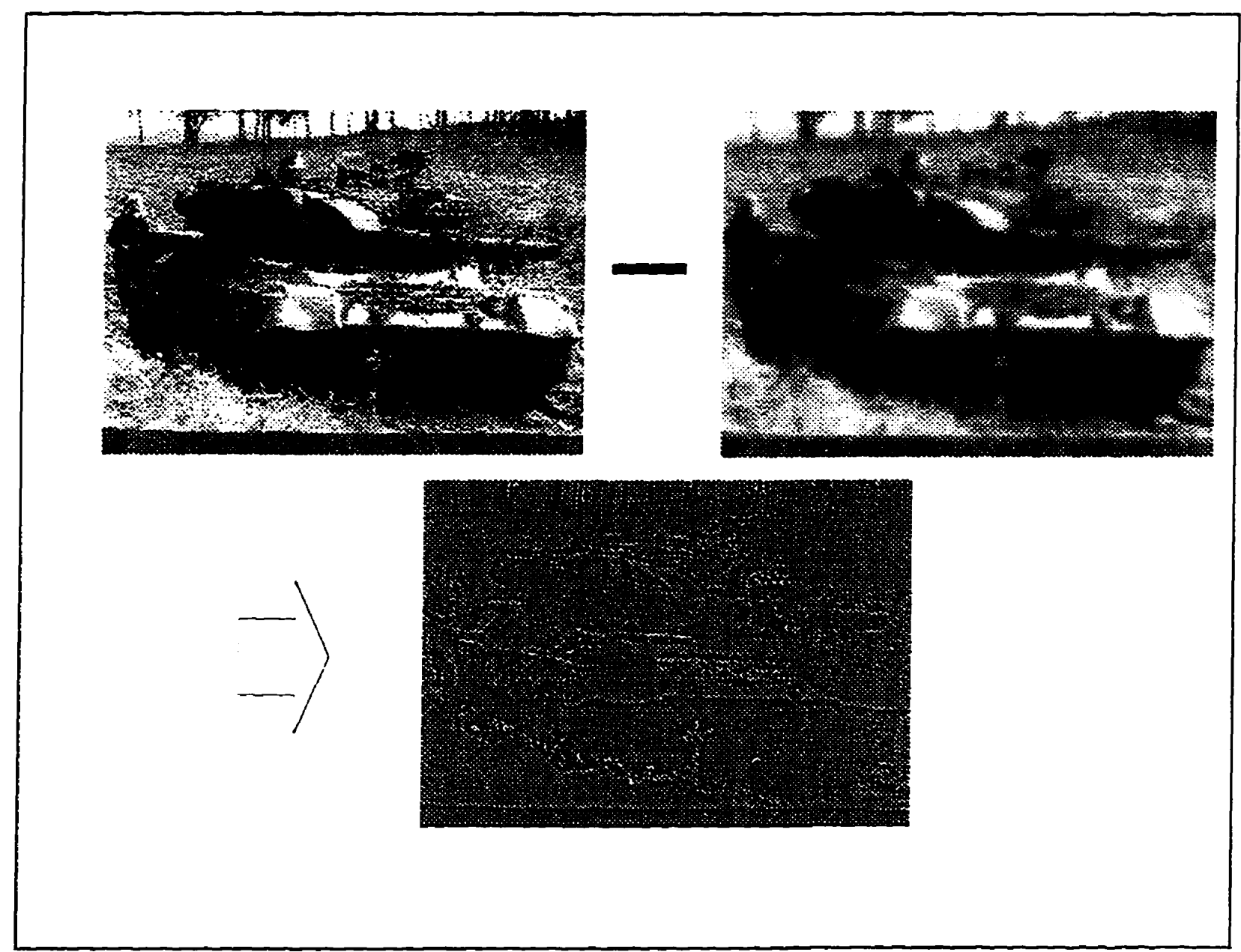

Figure 3-5 Sharpening Using a Low Pass Filter

direction chosen at all. For example, taking the derivative in the i direction (East or West) will optimally detect edges which are aligned vertically ( $j$ direction). The result of such convolutions will be an array of numbers where larger numbers represent edges of greater steepness and, when displayed, will either be a darker black (where white is the background) or brighter white (where black is the background) in the edge map depending on the photo interpretation of the image (see Chapter Four for a discussion of photo interpretation). As an example of a first derivative filter, consider a pixel and its eight neighbours as shown in figure 3-6. The i-derivative of the image at point $(i, j)$ can be determined using either 


$$
\begin{aligned}
f^{\prime}(i, j)= & f(i+1, j)-f(i, j) \\
& \text { or } \\
f^{\prime}(i, j)= & f(i, j)-f(i-1, j)
\end{aligned}
$$

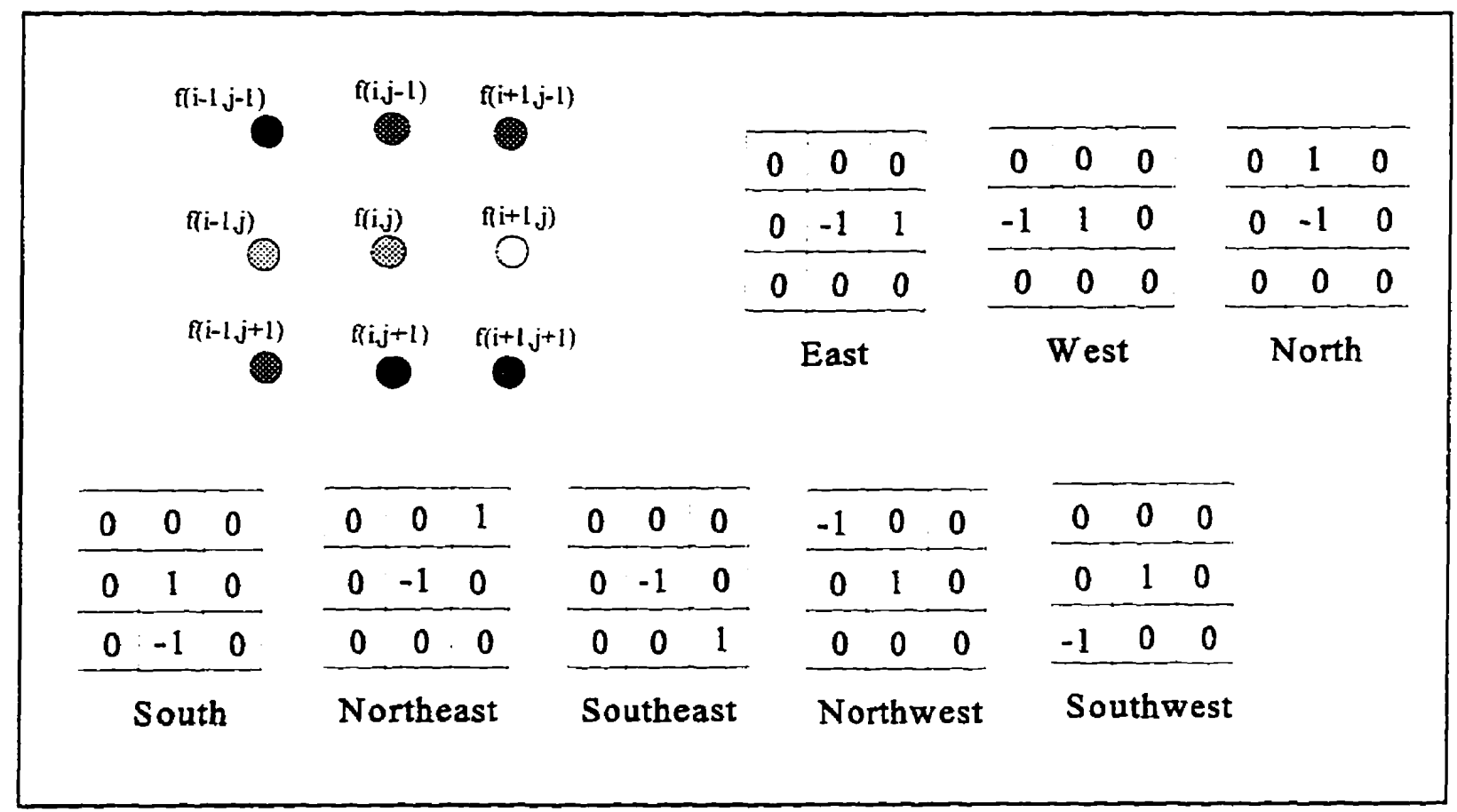

Figure 3-6 Examples of First Derivative Filters

The convolutional masks that would be used to determine such a derivative at each non-boundary pixel are also shown in figure 3-6, as well as the masks for derivatives in the other directions [Galb90].

Another option for detecting edges is analogous to the definition of gradient in calculus in that the operators are based on taking a difference in grey levels in two orthogonal directions and then calculating the magnitude and direction of the results [Gonz79]. Recall that, for a 
function $f(\mathrm{x}, \mathrm{y})$, the gradient at $(\mathrm{x}, \mathrm{y})$ is defined as the vector

$$
\nabla[f(x, v)] \quad\left[\begin{array}{l}
\frac{\partial f}{\partial x} \\
\frac{\partial f}{\partial y}
\end{array}\right]
$$

where the magnitude and direction of the gradient are given by

$$
\begin{gathered}
|\nabla[f(x, v)]| \sqrt{\left(\frac{\partial f}{\partial x}\right)^{2}+\left(\frac{\partial f}{\partial y}\right)^{2}} \\
\text { and } \\
\alpha=\tan ^{-1}\left[\frac{\left(\frac{\partial f}{\partial y}\right)}{\left(\frac{\partial f}{\partial x}\right)}\right.
\end{gathered}
$$

Once the gradient for each pixel in the image is determined, the user can then look for gradient magnitudes which are maximal in any of the eight directions and mark these pixels with a value based on their gradient magnitude within the output edge map. To illustrate this concept, consider a three dimensional representation of an image where the $i$ and $j$ axis provide spatial information and the vertical axis represents gray level values. The resulting graph resembles a topographical display of hills, plains and valleys. If a person could traverse these contours it would become quickly evident that some of the hills and valleys would be steeper (higher gradient) than others. These areas are where the stronger edges occur. If one could then measure the greatest rate of change at each step (pixel) through these hills and valleys, and the direction of greatest change $(\alpha)$, then one would have a means of recording, for each pixel, the gradient 
at that point. A more precise edge map is obtained when the technique described above is amended to include a stage during which the direction of maximal change is compared against the angle $\alpha$ determined in the gradient calculation. If these angles do not match within a predetermined tolerance (for example, $\pm 22.5^{\circ}$ if the number of possible directions is eight) then the pixel is not considered as an edge point.

There are a number of other approaches based on the gradient technique covered in the literature. These include such operators as the Sobel operator [Lind91] [Gonz79] [Ekst84] [Galb90], Prewitt operator [Hara84] [Nibl86] [Ekst84], and Roberts operator [Hara84] [Nibl86] [Ekst84] [Galb90]. The reader is directed to the references for a further discussion of these techniques.

Another approach is to use the Laplacian to create the edge operator. This approach detects intensity changes based on zero crossings of the second derivative of the image function (which corresponds to maxima in the first derivative). It is based on the second partial derivative Laplacian expression used with continuous functions:

$$
\nabla^{2} f=\frac{\partial^{2} f}{\partial x^{2}}+\frac{\partial^{2} f}{\partial y^{2}}
$$

In the discrete case, the Laplacian measures the change in slope of the intensity at a point (i,j) in the $\mathrm{i}$ and $\mathrm{j}$ directions as follows: 


$$
\begin{array}{lc}
\text { Continuous } & \text { Discrete } \\
f(x, y) & f(i, j) \\
\frac{\partial f}{\partial x} & f\left(i+\frac{1}{2}, j\right)-f\left(i-\frac{1}{2}, j\right) \\
\frac{\partial f}{\partial y} & f\left(i, j+\frac{1}{2}\right)-f\left(i, j-\frac{1}{2}\right) \\
\frac{\partial^{2} f}{\partial x^{2}} & {[f(i+1, j)-f(i, j)]-[(f(i, j)-f(i-1, j)]} \\
\frac{\partial^{2} f}{\partial y^{2}} & {[f(i, j+1)-f(i, j)]-[(f(i, j)-f(i, j-1)]} \\
\frac{\partial^{2} f}{\partial x^{2}}+\frac{\partial^{2} f}{\partial y^{2}} & f(i+1, j)+f(i-1, j)+f(i, j+1)+f(i, j-1)-4 f(i, j)
\end{array}
$$

The discrete expression for the Laplacian is an approximation of the continuous Laplacian and can be represented as a convolution kernel [Lind91]:

$$
\begin{array}{ccc}
0 & 1 & 0 \\
1 & -4 & 1 \\
0 & 1 & 0
\end{array}
$$

This mask is isotropic in nature in that it provides only scalar information regarding the 
magnitude of intensity shifts. The negative of this mask is generally used as the discrete Laplacian even though this is actually the negative of the mathematical Laplacian [Nibl86]. When this mask is convolved with an image, the points where the Laplacian changes from negative to positive or vice-versa indicate the points of greatest intensity change within the image. In order to derive more information from the process, some authors have suggested looking at the slope of the zero crossings of the Laplacian to provide information on the relative strength of edges [Marr80] [Hara84].

Another rendition of these themes was studied by Robert M Haralick. In his approach. Haralick used the direction of the gradient and the zero crossings of the directional second derivative (as apposed to the scalar Laplacian) to locate edges [Hara84]. He concluded that this approach is superior to the Prewitt operator and Laplacian approaches. In addition to this technique and the ones discussed above, there are a number of other techniques used in the detection of edges. All share the same characteristic that the gray levels of a pixel's neighbours are used to determine whether an edge exists based on rates of change in intensity. They include techniques such as scale-space filtering [Xin95], steerable filters [Free91], "fuzzy reasoning" [Law96], Hermite Integration [Shen95], and Gabor filtering [Mehr92] [Namu94][Buf90]. Further explanations of these techniques can be found in the referenced articles should the reader wish to explore these in more depth.

With regards to gradient and Laplacian operators, further research into these techniques has led to the development of a procedure for limiting the effects of noise during the convolution operation. A person could simply median filter or smooth an image prior to edge processing; however, while smoothing has long been known to have the benefit of reducing noise, it has the 
inherent negative side-effect of degrading positional accuracy. In effect, the stronger the degree of smoothing that is employed, the greater the noise reduction and the greater the loss in positional accuracy. In the late 1970s, D. Marr and E Hildreth began a detailed study into the idea of smoothing the image prior to implementing a Laplacian based edge detector [Marr80]. They found that by convolving an image with a Gaussian and simultaneously determining the Laplacian of the output, very good noise reduction results were obtained with spatial coincidence from two or more channels (ie different Gaussian standard deviations) indicating an edge segment based on a single physical phenomena. In the mid-1980s, John Canny undertook to study the effects of smoothing an image with various smoothing filters prior to gradient based edge detection. In his paper "A Computational Approach to Edge Detection," (1986) Canny proved that the Gaussian was optimal for smoothing an image in a way that reduces the effects of noise while minimizing the consequent loss of positional accuracy. In using this technique, as with Marr, the standard deviation of the Gaussian ( $\sigma)$ is taken as a measure of scale or resolution as it controls both the degree of smoothing applied to the image and the consequent loss of positional accuracy. Hence, the smaller the required $\sigma$ value, the better the localization of the results. Previously, in 1984, Valdis Berzins conducted a study which demonstrated that the accuracy of Laplacian edge detectors could be kept within $1.4 \sigma$ in most circumstances. To further illustrate this, consider a $100 \times 100$ pixel image of a photo of two parallel lines and another image of the same two lines blown up to twice the size of the first (200x200). If one applies Canny's edge detector with a resolution of $\sigma_{1}$ to the $100 \times 100$ image, and then applies the exact same filter to the $200 \times 200$ image, a measurement of the distance between the two lines in the second case will be closer to their true separation than in the first case. Thus in attempting to 
take measurements on real-world images, it is important to keep the scale of the filter $(\sigma)$ low and use images which have a high resolution (pixels per inch). In real applications, a user must consider the trade-off between improving the quality of measurements within a resulting edge map against the time that it takes to process larger images.

There are a considerable number of examples within the literature involving this Gaussian smoothing edge detection operation including [Will90], [Deri93], [Talt85], [Hart85], [Berg84], [Korn88], [Hild83], [Neyc93], [Berg87], [Torr86], [Berz84] and [Chen89]. They involve the use of both directionally sensitive gradient operators and omnidirectional Laplacian operators. As part of this study, one particular technique from each of the research papers done on these two types of operators was selected in order to implement edge detection algorithms within the application program. The first is based on Franck Neycenssac's article "Contrast Enhancement Using the Laplacian-of-a-Gaussian Filter' and the second on Axel F. Korn's article "Toward a Symbolic Representation of Intensity Changes in Images." An explanation of the mathematics behind these algorithms and an illustration of their effect will be provided in the following two sections of this chapter.

\section{3-4 Laplacian of a Gaussian Filter}

As its name implies, the LoG filter is based on a process which takes the Laplacian of an image which has been smoothed through convolution with a Gaussian. The great advantage of this technique is that the Laplacian operator $\left(\nabla^{2}\right)$ is orientation independent. This simplifies the procedure by limiting the edge detection process to one convolution for any given scale ( $\sigma$ ) 
instead of having to determine zero crossings in the direction of the gradient for the seconddirectional derivative of a function [Marr80]. In Franck Neycenssac's article, he uses the technique presented by Marr and Hildreth [Marr80] which uses a discrete convolution mask to approach the ideal continuous LoG filter. The technique involves:

1. computing the LoG filter to obtain a convolution kernel,

2. convolving the image with this filter to obtain a new image $L$,

3. multiplying this new image by a display factor, $\beta$ to obtain $\beta \mathrm{L}$, and

4. subtracting $\beta \mathrm{L}$ from the original image I to obtain an enhanced image I - $\beta \mathrm{L}$.

In this section, we will only explore the first two stages as the final two are designed to enhance the contrast within an image and we are interested only in the edge detection process. Nonetheless, using the multiplication and subtraction capabilities of the program (see Chapter Four), it is possible to use the application program in its current state to perform the final two steps if required by a user.

The first step then is to obtain the convolution filter which will perform the smoothing and edge detection functions. The process can be summarized mathematically by first studying the continuous case and then the discrete equivalent. We begin with the definition of a LoG filter:

$$
L o G=\nabla^{2}(I * G(r, \sigma))
$$

where 


$$
\begin{gathered}
G(r, \sigma)=\frac{1}{2 \pi \sigma^{2}} e^{\frac{r^{2}}{2 \sigma^{2}}} \\
o r \\
G(x, y, \sigma)=\frac{1}{2 \pi \sigma^{2}} e^{-\frac{x^{2}-y^{2}}{2 \sigma^{2}}}
\end{gathered}
$$

Recalling that convolution is associative, ie

$$
\frac{d}{d t}[f * g]=f^{\prime} * g=f * g^{\prime}
$$

we have

$$
\nabla^{2}(I * G(r, \sigma))=I * \nabla^{2} G(r, \sigma)
$$

It is then only necessary to calculate $\nabla^{2} \mathrm{G}(r, \sigma)$, which is, by definition :

$$
\nabla^{2} G(x, y, \sigma)=\frac{\partial^{2} G(x, \nu, \sigma)}{\partial x^{2}}+\frac{\partial^{2} G(x, \nu, \sigma)}{\partial y^{2}}
$$

then

$$
\frac{\partial}{\partial x} G(x, y, \sigma)=\frac{1}{2 \pi \sigma^{2}}\left(\frac{-x}{\sigma^{2}}\right) e^{-\frac{x^{2} \cdot y^{2}}{2 \sigma^{2}}}
$$

Similarly, 


$$
\begin{aligned}
\frac{\partial^{2}}{\partial x^{2}} G(x, y, \sigma) & =\frac{-1}{2 \pi \sigma^{4}} e^{-\frac{x^{2} \cdot y^{2}}{2 \sigma^{2}}}-\frac{x}{2 \pi \sigma^{4}}\left(\frac{-x}{\sigma^{2}}\right) e^{-\frac{x^{2}-y^{2}}{2 \sigma^{2}}} \\
& =\frac{-1}{2 \pi \sigma^{4}}\left[1-\frac{x^{2}}{\sigma^{2}}\right] e^{-\frac{x^{2} \cdot y^{2}}{2 \sigma^{2}}} \\
\frac{\partial^{2}}{\partial y^{2}} G(x, y, \sigma) & =\frac{-1}{2 \pi \sigma^{4}}\left[1-\frac{y^{2}}{\sigma^{2}}\right] e^{-\frac{x^{2} \cdot y^{2}}{2 \sigma^{2}}}
\end{aligned}
$$

Hence, combining equations $3.2,3.5$ and 3.6 we have

$$
\nabla^{2} G(x, \nu, \sigma)=\frac{\partial^{2}}{\partial x^{2}} G(x, \nu, \sigma)+\frac{\partial^{2}}{\partial y^{2}} G(x, \nu, \sigma)=\frac{-1}{2 \pi \sigma^{4}}\left[1-\frac{x^{2}+y^{2}}{2 \sigma^{2}}\right] e^{-\frac{x^{2} \cdot y^{2}}{2 \sigma^{2}}}
$$

To create the necessary convolutional kernel, we simply need to sample equation 3.7 and introduce a sampling factor $\mathrm{k}$ :

$$
L o G=\nabla^{2} G(x, y, \sigma)=\frac{-1}{2 \pi \sigma^{4}}\left[1-k \cdot \frac{x^{2}+y^{2}}{2 \sigma^{2}}\right] e^{-\frac{x^{2} \cdot y^{2}}{2 \sigma^{2}}}
$$

The sampling factor $\mathrm{k}$ should allow us to conserve the Laplacian property that

$$
\int_{0}^{2 \pi} \int_{0}^{+\infty} L o G(r) \cdot r \cdot d r \cdot d \Theta=0
$$


This is the mathematical form of the property discussed earlier for smoothing filters - the sum of the coefficients within a smoothing mask is zero. As $k-1$ this property is upheld. If we set the size of the kernel at an odd integer $M$, define $M=2 h+1$ and substitute $i$ for $x$ and $j$ for $y$, equation 3.9 is written in discrete form as the property [Neyc93]

$$
\sum_{j=-h}^{j=h} \sum_{i=-h}^{i=h}\left(1-k \frac{i^{2}-j^{2}}{2 \sigma^{2}}\right) \cdot e^{-\frac{i^{2}-y^{2}}{2 \sigma^{2}}}=0
$$

Rearranging this formula we obtain $k=\sigma^{2} \cdot A / B$, where

$$
\begin{gathered}
A=\sum_{i=-h}^{i=h} e^{\frac{-i^{2}}{i a^{2}}} \\
\text { and } \\
B=\sum_{i=-h}^{i=h} i^{2} \cdot e^{\frac{-i^{2}}{i^{2}}}
\end{gathered}
$$

We have then that the larger the value of $M$ and $\sigma$, the closer that $k$ gets to the optimal case of $k=1$. In Neycenssac's approach, the value of $h$ is set $\geq 4 \sigma$ (therefore $M \geq 8 \sigma+1$ ) to allow sufficient precision with the detection of zero crossings. In this case, $k<1.001$. Korn's approach roughly agrees with this although he uses the requirement that the mask is cut off when the side lobes drop below $5 \%$ of the centre maximum value - which corresponds to an $\mathrm{h}$ value of approximately $3 \sigma$. In Korn's case, if a $\sigma$ value of 1.0 is chosen, then $h=3, M=7$ and the correspondence factor $(\mathrm{k})$ is calculated as follows: 


$$
\begin{aligned}
& k=\sigma^{2} \cdot \frac{A}{B} \\
& \text { where } \\
& A=\sum_{i=-3}^{i=3} e^{\frac{-i^{2}}{2+(1.0)^{2}}} \\
& =2 e^{\frac{-9}{2}}+2 e^{\frac{s}{2}}+2 e^{\frac{-1}{2}}+e^{0} \\
& =2 e^{-4.5}+2 e^{-2}+2 e^{-0.5}+1 \\
& =2.5059499 \\
& \text { and } \\
& B=\sum_{i=-3}^{i=3} i^{2} e^{\frac{-i^{2}}{2-11.0 r^{2}}} \\
& =2(9) e^{\frac{-4}{2}}+2(4) e^{\frac{-4}{2}}+2(1) e^{\frac{-1}{2}}+(0) e^{0} \\
& =18 e^{-4.5}+8 e^{-2}+2 e^{-0.5}+0 \\
& =2.4957056 \\
& k=(1.0)^{2} \cdot \frac{2.5059499}{2.4957056} \\
& =1.0041048
\end{aligned}
$$

This is well below the limit of 1.1 suggested by Neycenssac [Neyc93]. The advantage of using a smaller kernel $(h<4 \sigma)$ is that much less CPU time is required to complete the convolution. As this can be done by using a $\sigma$ value $\geq 0.5$ and an $h$ value of $3 \sigma$ without compromising the accuracy of the discrete model, this is preferable.

As a means of demonstrating this technique, the final part of this section provides an 
example of a LoG filtering of a small image. In figure 3-7, a small section (20x20) of an image is selected to demonstrate the process. Note that this image has a photo_interpretation of 1: therefore, 255 is white and 0 is black. We select a $\sigma$ value of 1.0 , set $\mathrm{h}=40$, and derive the convolutional kernel as shown in figure 3-8. To illustrate the effects of $\sigma$ on the correspondence factor, the following are the sums of the kernel coefficients for this case and several other $\sigma$ (ideally $\sum_{\mathrm{i}} \sum_{\mathrm{j}}=0$ ):

$$
\begin{array}{ll}
\sigma=0.6 & \sum_{\mathrm{i}} \sum_{\mathrm{j}}=0.1297134389 \\
\sigma=0.7 & \sum_{\mathrm{i}} \sum_{\mathrm{j}}=0.0099633998 \\
\sigma=0.8 & \sum_{\mathrm{i}} \sum_{\mathrm{j}}=0.0007943 \\
\sigma=1.0 & \sum_{\mathrm{i}} \sum_{\mathrm{j}}=0.00014400457 \\
\sigma=3.0 & \sum_{\mathrm{i}} \sum_{\mathrm{j}}=0.000117404831
\end{array}
$$

It is apparent then that, indeed, as $\sigma$ gets larger, the size of the convolution kemel increases which together result in a closer approximation of the continuous LoG filter.

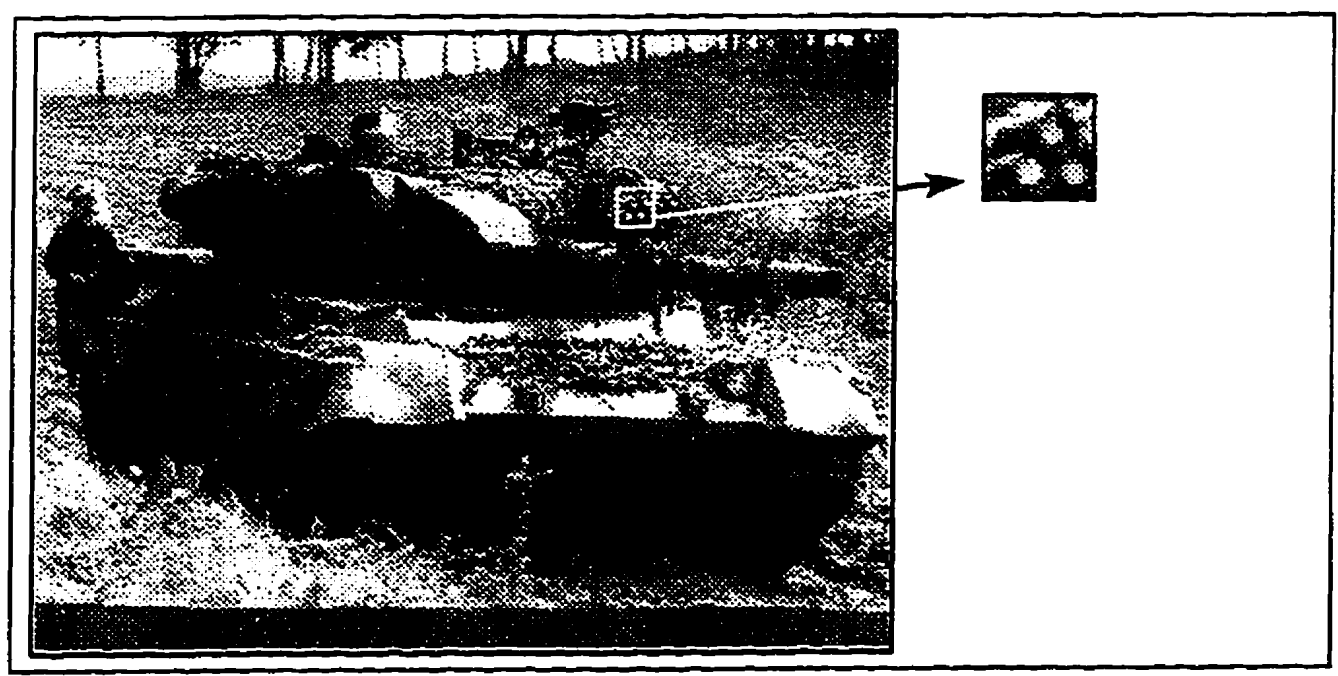

Figure 3-7 Image Selected for Edge Detection Example 
1\|\|\|\|

\|\|\|\|$\|$

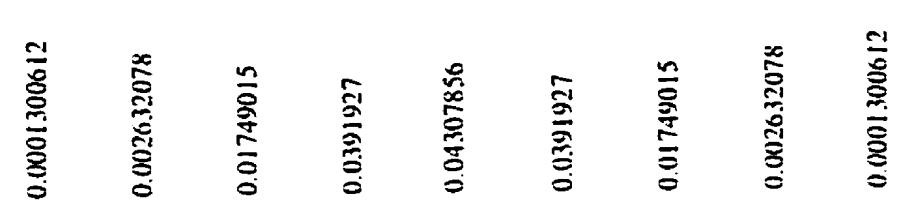

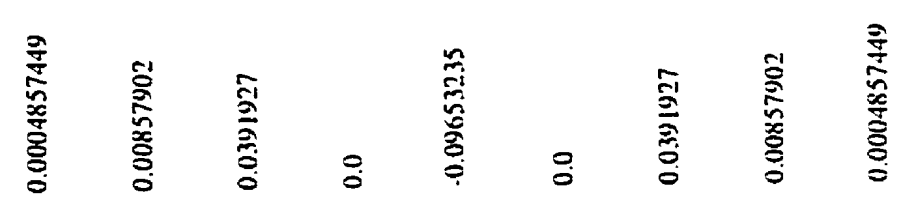

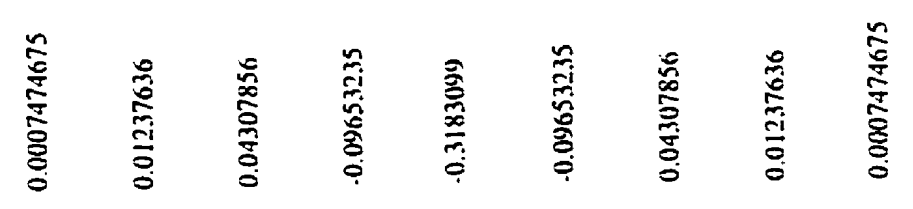

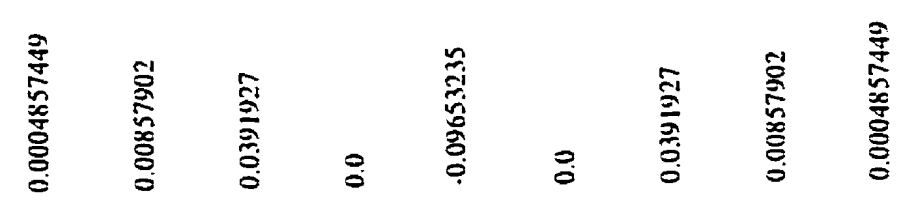

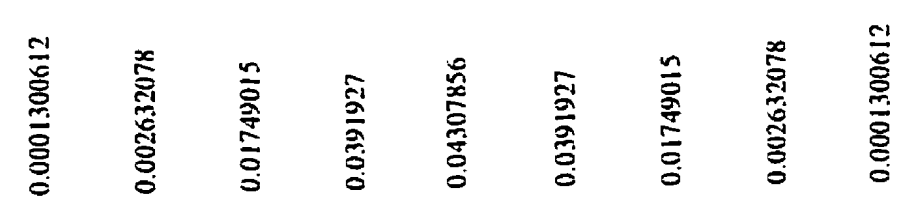

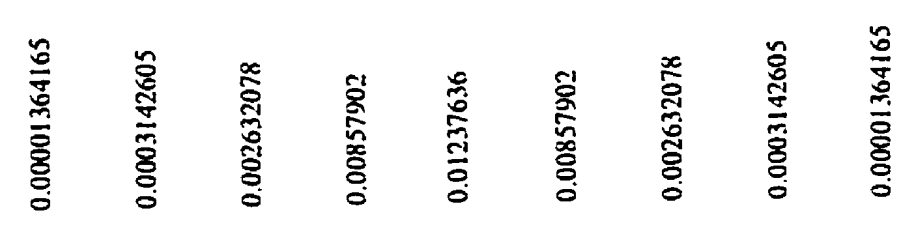

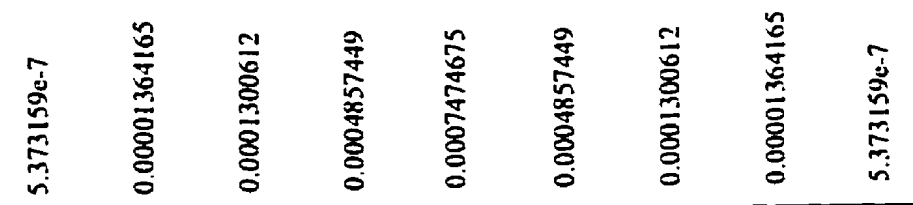




\section{Original lmage Pixel Values:}

$\begin{array}{llllllllllllllllllll}129 & 106 & 138 & 148 & 151 & 135 & 137 & 112 & 154 & 145 & 125 & 113 & 123 & 114 & 164 & 188 & 107 & 73 & 93 & 199\end{array}$

$\begin{array}{lrllllllllllllllllll}129 & 97 & 132 & 131 & 131 & 198 & 215 & 230 & 204 & 222 & 243 & 227 & 155 & 93 & 175 & 253 & 177 & 151 & 162 & 225\end{array}$

$\begin{array}{llllllllllllllllllll}123 & 101 & 121 & 157 & 217 & 230 & 251 & 255 & 255 & 249 & 247 & 194 & 181 & 102 & 96 & 194 & 220 & 240 & 249 & 225\end{array}$

$\begin{array}{llllllllllllllllllll}133 & 135 & 127 & 186 & 201 & 189 & 242 & 243 & 245 & 230 & 200 & 83 & 116 & 36 & 44 & 154 & 247 & 241 & 231 & 99\end{array}$

$\begin{array}{llllllllllllllllllll}204 & 209 & 235 & 240 & 252 & 243 & 234 & 214 & 197 & 167 & 126 & 155 & 181 & 50 & 40 & 39 & 168 & 188 & 202 & 160\end{array}$

$\begin{array}{llllllllllllllllllll}254 & 247 & 242 & 226 & 223 & 194 & 160 & 120 & 108 & 123 & 195 & 201 & 212 & 57 & 30 & 17 & 76 & 139 & 168 & 219\end{array}$

$\begin{array}{llllllllllllllllllll}241 & 235 & 167 & 133 & 117 & 93 & 84 & 90 & 75 & 114 & 194 & 211 & 220 & 192 & 61 & 28 & 86 & 200 & 218 & 214\end{array}$

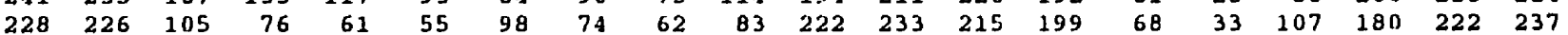

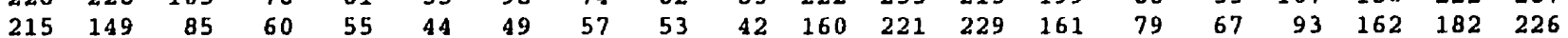

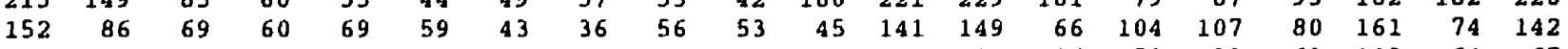

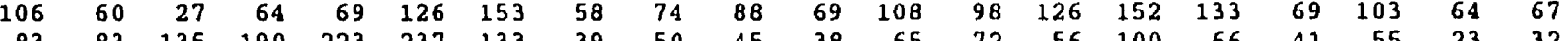

$\begin{array}{rrrrrrrrrrrrrrrrrrrr}83 & 83 & 135 & 190 & 223 & 237 & 133 & 39 & 50 & 45 & 38 & 65 & 72 & 56 & 100 & 66 & 41 & 55 & 23 & 32\end{array}$

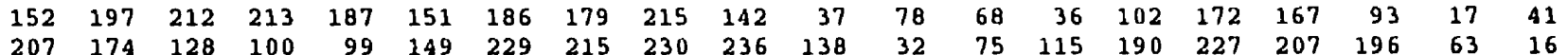

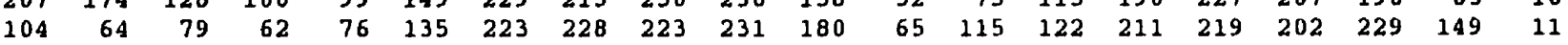

$\begin{array}{llllllllllllllllllll}114 & 69 & 42 & 36 & 42 & 102 & 208 & 236 & 227 & 231 & 186 & 73 & 121 & 77 & 169 & 222 & 227 & 222 & 165 & 49\end{array}$

$\begin{array}{rlllllllllllllllllll}49 & 62 & 40 & 30 & 56 & 66 & 219 & 226 & 220 & 181 & 88 & 108 & 163 & 33 & 72 & 185 & 218 & 206 & 81 & 80\end{array}$

white $=255$

black $=0$

\section{Pixel Values after Convolution with LoG Filter:}

\begin{tabular}{|c|c|c|c|c|c|c|c|c|c|c|c|}
\hline .43 .49230 & -36.81252 & -30.93494 & $\cdot 20.0 \% 196 \%$ & .6 .73911 & H. 11010 & 19.62933 & 1.99943 & .3 .81423 & 49.61304 & 14.82521 & A1.6689H \\
\hline$-3 \%, 30968$ & .22 .89906 & $-2.816 \% 3$ & 18.13080 & 32.22032 & 23.19734 & $\because .5 \% 344$ & .36 .88964 & .10 .02596 & 19.30991 & $6 \% .11683$ & $13.1262 \mathrm{H}$ \\
\hline $26.451 \%$ & 21.12607 & 20.47131 & 28,12610 & 40.80797 & 19.09350 & $.31 .89 \% 0$ & . $32.79 \% 00$ & -58.24871 & -28.03258 & 31.75266 & 6b.3786' \\
\hline $40.420 \% 9$ & 32.00470 & 12.8650ذ & 14.33716 & 34.24762 & 19.28459 & .43 .36318 & $.68 .5329 \%$ & $.6 \% .92191$ & .11 .78598 & 27.02624 & 34.680 .26 \\
\hline 33.29411 & 31.41593 & 22.25680 & 19.96913 & $32.5654 \mathrm{~A}$ & 33.88655 & . 13.34824 & .59 .60840 & $.5 \% .25294$ & -16.22468 & $23.8 \% 002$ & 36.10064 \\
\hline $31.31 / 59$ & 22.22180 & 20.76484 & $26.16 \% \mathrm{ss}$ & 23.90916 & 31.76996 & 22.23734 & .16 .84364 & $.1 \% .64233$ & 8.99911 & 4.01948 & 3. $2 \mathrm{~s} \in 000$ \\
\hline $5.066 \% 1$ & .21 .82473 & 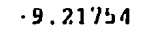 & $31.10 \% 1$ & $2 \% .271433$ & 22.6232 .2 & 26.0692 .2 & $4.6 \% 2.99$ & 1.93919 & 1.66011 & -19.43970 & - 1.63185 \\
\hline .39 .52037 & $.5 \%, 3804 \%$ & $-13,086 \%$ & 38.315157 & 34.10166 & $33.9 \% 08 \%$ & 42.84093 & 22. 26, & 20.90507 & 28.52 .469 & 12.52511 & 16.941111 \\
\hline .38 .61521 & .26 .16310 & .20 .39088 & 14.32179 & -29.15060 & $.9 .23 \% 43$ & 35.46333 & 36.36403 & 33.52140 & $40.696 \mathrm{~b} 1$ & 8.91250 & 21.290981 \\
\hline $22.47 \% 7$ & 1.31557 & .35 .89779 & $.43 .2 \% 948$ & $.5 \% .4 \% 0 \%$ & 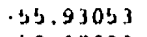 & $-0,31260$ & 42.70465 & 29.335366 & $5.2 \% 0 \% 4$ & -36.71349 & 19.629\% د. \\
\hline 12.52 .170 & 7.30107 & -38.86801 & -40.23120 & $.12,12273$ & 18293. 1829. & 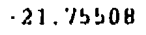 & 31.97304 & $1 \% .25 \% 46$ & $\cdot 8.0 \leq 22.2$ & $.46 .8208 \%$ & $.5 \% .6442 \%$ \\
\hline 47.82934 & 15.11169 & .14 .23979 & . & داد 18,366ذ. & $.4 \% .89151$ & $\cdot 12.60931$ & 18.2182 .5 & $3.45 \% 7 \%$ & 15.13159 & $.1 \% .74 \% 80$ & SH, SH/HA? \\
\hline
\end{tabular}

Figure 3-9 Example of LoG Convolution 
The pixel values of the area selected and the results of convolution are summarized in figure 3-9. Note that the size of the pixel array decreases by four rows and four columns as a result of the loss of information around the edges due to convolution ( $M=9)$.

Once the convolution is complete, the resulting pixel values are comprised of both positive and negative values. These can be normalized to the range $0-255$ for display purposes where a 0 becomes an intermediate gray value. The result of applying this technique to the previous example is provided in figure 3-10. To find the edges within the mapping, a program can first convert all the negative values of the original convolution result to black and all positive values to white (or vice-versa). Edges are then found by searching for any pixel which has a neighbour which is of the opposite colour [Neyc93].

\begin{tabular}{|rrrrrrrrrrrr}
\hline 40 & 56 & 66 & 86 & 109 & 136 & 156 & 132 & 112 & 210 & 254 & 201 \\
55 & 81 & 116 & 154 & 179 & 163 & 108 & 56 & 50 & 156 & 242 & 252 \\
151 & 159 & 158 & 171 & 194 & 155 & 65 & 27 & 18 & 72 & 189 & 238 \\
193 & 178 & 144 & 147 & 182 & 156 & 44 & 0 & 1 & 47 & 169 & 219 \\
181 & 177 & 161 & 157 & 179 & 182 & 94 & 15 & 20 & 93 & 167 & 186 \\
177 & 161 & 158 & 169 & 164 & 178 & 161 & 91 & 90 & 137 & 129 & 127 \\
130 & 83 & 105 & 177 & 171 & 162 & 168 & 130 & 125 & 124 & 87 & 113 \\
16 & 19 & 98 & 190 & 182 & 182 & 198 & 161 & 159 & 172 & 142 & 152 \\
53 & 75 & 85 & 96 & 69 & 105 & 184 & 186 & 181 & 194 & 137 & 84 \\
161 & 124 & 58 & 44 & 19 & 22 & 121 & 197 & 174 & 131 & 56 & 15 \\
197 & 134 & 52 & 50 & 45 & 16 & 83 & 178 & 152 & 107 & 38 & 19 \\
206 & 149 & 43 & 23 & 35 & 36 & 99 & 154 & 131 & 148 & 90 & 18 \\
& & & & & & & & & & \\
\hline
\end{tabular}

Figure 3-10 Normalized Pixel Values after LoG Filtering

In order to make this process even more useful, it is possible to determine the slope of 
the zero crossings and use these as a measure of the relative strength of the contour at that point. The steeper the slope, the larger the contrast between pixels in the original image. This would then allow a user to use histogram techniques to remove weaker contours and/or strengthen others. Through this process it could be easier to emphasize certain features within an image. An exploration of this technique has not been done here although it would be a good selection for an area of future study. However, the next process to be discussed involving the Derivative of a Gaussian (DoG) filter does incorporate an edge-strength capability as will be shown in the next section.

\section{3-5 Derivative of a Gaussian Filter}

The second edge detection technique that was selected is the DoG filter. The technique described below is based on that used by Dr Axel F Korn as described in his article "Toward a Symbolic Representation of Intensity Changes in Images" (1988). It involves using the gradient of the Gaussian smoothed image function to create an "edge map" file for an image. The $x$ and $y$ components of the image gradient are determined by taking the partial first derivative of a twodimensional Gaussian in both the $\mathrm{x}$ and $\mathrm{y}$ directions and then convolving these with the subject. Recall that, by the associative property of convolution, we have

$$
\nabla(f(x, y) * G(x, v, \sigma))=f(x, v) * \nabla G(x, y, \sigma)
$$

It is then only necessary to calculate $\nabla \mathrm{G}(\mathrm{x}, \mathrm{y}, \sigma)$ and convolve this with the subject image. Recall from earlier discussions that the two dimensional Gaussian function with standard deviation $\sigma$ is given by 


$$
G(x, y, \sigma)=\frac{1}{2 \pi \sigma^{2}} e^{-\frac{x^{2}-y^{2}}{2 \sigma^{2}}}
$$

The partial first derivatives of the Gaussian function are thus given by

$$
\begin{gathered}
\text { continuous } \\
G_{x}=\frac{\partial G}{\partial x}=-\frac{x}{2 \pi \sigma^{4}} e^{-\frac{x^{2} \cdot y^{2}}{2 \sigma^{2}}} \\
G_{y}=\frac{\partial G}{\partial y}=-\frac{y}{2 \pi \sigma^{4}} e^{-\frac{x^{2} \cdot y^{2}}{2 \sigma^{2}}} \\
G_{i}=-\frac{i}{2 \pi \sigma^{4}} e^{-\frac{i^{2}, y^{2}}{2 \sigma^{2}}} \quad(3.11) \\
G_{j}=-\frac{j}{2 \pi \sigma^{4}} e^{-\frac{i^{2}, y^{2}}{2 \sigma^{2}}} \quad(3.12) \\
\text { where } \\
i, j \in I \quad \text { and }-\frac{(M-1)}{2} \leq i \leq \frac{M-1}{2} \\
-\frac{(N-1)}{2} \leq j \leq \frac{N-1}{2}
\end{gathered}
$$

As discussed previously, the standard deviation, $\sigma$, of the Gaussian can be considered as a measure of resolution as varying this quantity alters the degree of smoothing and consequently the amount of edge points that are detected.

In Korn's technique, he uses the sum of positive valued pixels of the filter to normalize 
the values of each coefficient such that they add up to +1.0 . The negative coefficients likewise add to -1.0 . He does this in order to compare gray value gradients for different resolutions. This aspect of the process is not investigated within this paper as a specific analysis of the effects of resolution changes were not included as part of our aim; however, the coefficients for $G_{x}$ and $G_{y}$ are adjusted in this manner in order to compare our results with those discussed in Korn's paper.

The window size, $\mathrm{MxN}$, is set such that the values less than 5 percent of the maximum of the operator are omitted. Using this criterion, for $\sigma=0.8$ the window size is $5 \times 5$ and for $\sigma=1.0$ the window size is $7 \times 5$. As discussed in the previous section on LoG filters, this equates to a correspondence factor, $\mathrm{k}$, of approximately $\mathrm{l} .004$ which is well within the limits discussed by Neycenssac [Neyc93]. Using this criterion, the formulae given in 3.11 and 3.12 and normalizing the results as discussed above, we can determine the convolution masks $G_{x}$ and $G_{y}$ for any value $\sigma$. For example, refer to figure 3-11 for the $G_{i}$ and $G_{j}$ filter masks for $\sigma=0.5$ and $\sigma=0.8$.

The complete DoG filter technique is summarized in the following algorithm:

1. The convolution $f^{*} \mathrm{G}_{\mathrm{i}}$ and $f^{*} \mathrm{G}_{\mathrm{j}}$ of an image $f(i, j)$ is carried out using kernels such as those provided in figure 3-11 for the $\sigma$ value selected;

2. These two convolutions create two pictures, $n_{1}(i, j, \sigma)$ and $n_{2}(i, j, \sigma)$ which represent the $i$ and $j$ components of the normal, $\mathbf{n}$, to the gradient of the original image at any point (i,j) within the non-border pixels. Amplitude and direction of $\mathbf{n}$ are thus defined by

$$
\begin{aligned}
& |\boldsymbol{n}|=\sqrt{n_{1}^{2}+n_{2}^{2}}=A(i, j, \sigma) \\
& \alpha=\tan ^{-1}\left(\frac{n_{2}}{n_{1}}\right)
\end{aligned}
$$




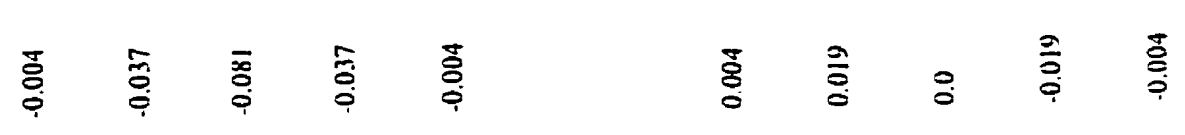

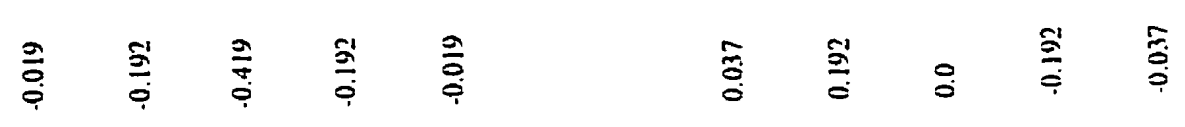

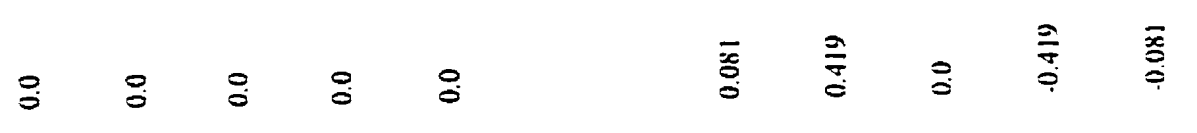

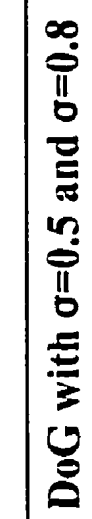

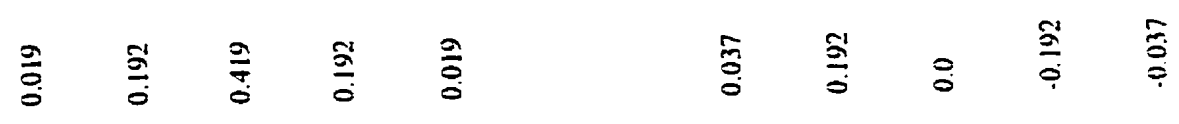

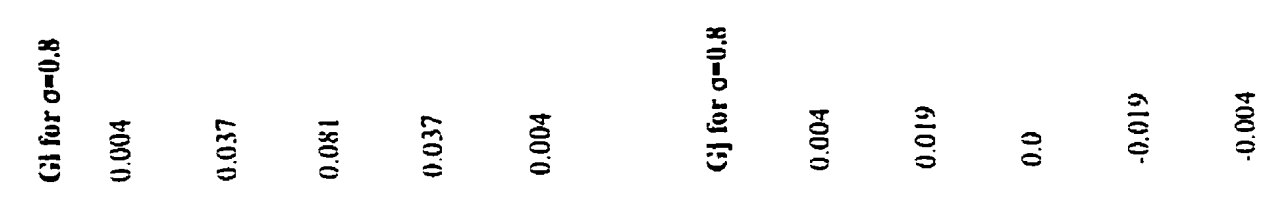

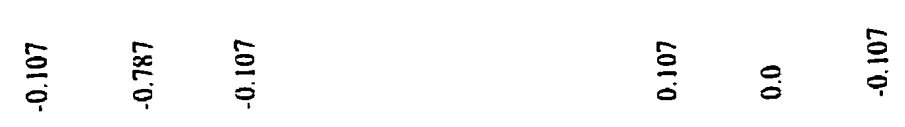

ะะะ

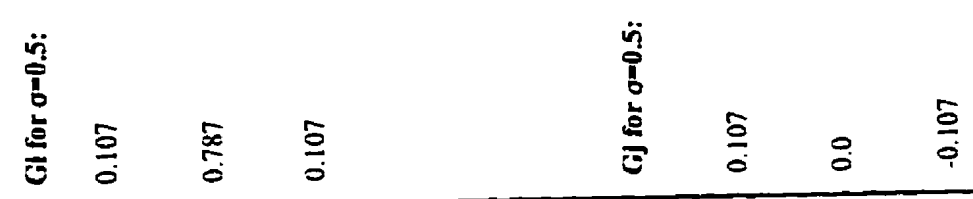




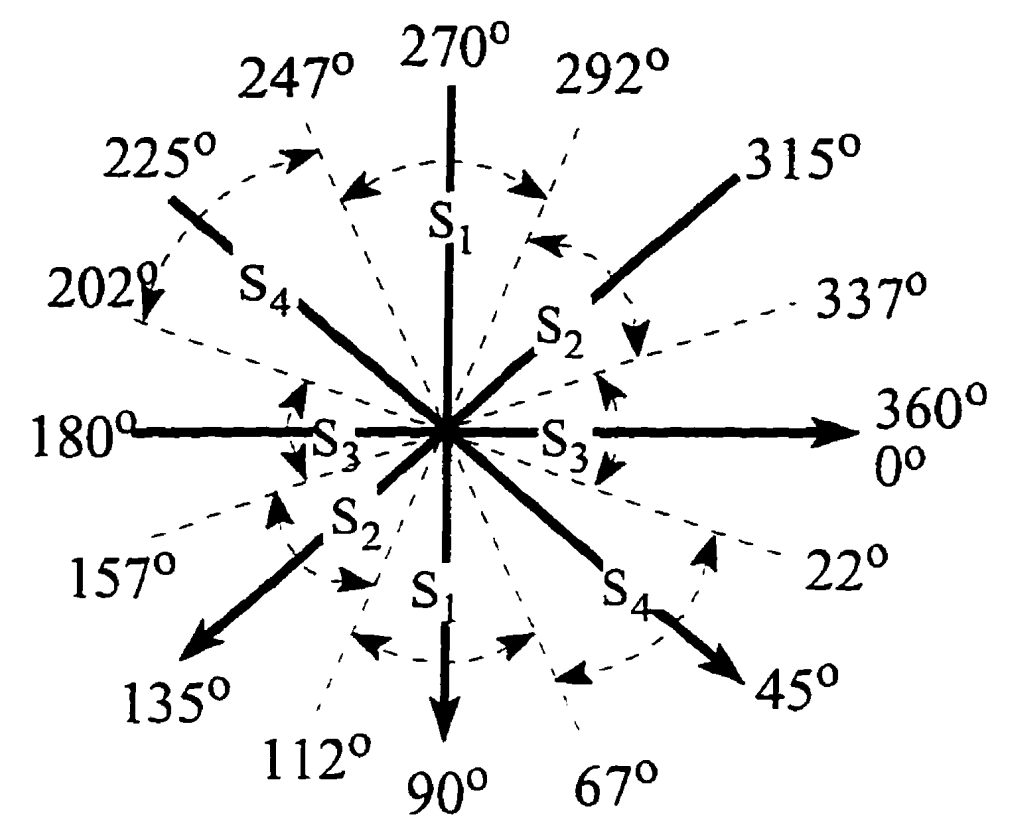

Figure 3-12 DoG Search Paths

Each point in the amplitude picture is then checked to determine if it is a maximum among its eight neighbouring amplitudes along four search paths: $0^{\circ}, 45^{\circ}, 90^{\circ}$ and $135^{\circ}$. Whenever a maximal candidate is encountered, its direction is compared against the direction $\alpha$ determined above. If the angle is within a tolerance of \pm $22.5^{\circ}$, this pixel is recorded as an edge point (see figure 3-12). For example, if a maxima is encountered in the positive i-direction within the amplitude array, then, for it to be an edge point, the corresponding angle " $\alpha$ " for that pixel would have to be between $337^{\circ}$ and $22^{\circ}$ or $157^{\circ}$ and $202^{\circ}$ (search path $S_{3}$ in figure $3-12$ ). If the angles do not match, the maximal point is recorded in a separate array which stores all maxima encountered. Also, all minima are recorded in the same fashion, irrespective of direction, in a separate array. These two arrays comprised of all 
maxima and all minima encountered are used later during the stage when holes in connected chains of edge points are encountered (see item 3 below); and

3. Discontinuities along the connected chains of the edge points, which are often caused by the difficulty with determining the exact location of gray value vertices, are filled by searching for a suitable "connector" from all maximal or minimal points amongst the gray value gradients - irrespective of the direction of the gradient. The procedure is:

i. First determine whether a discontinuity exists. Two factors determine that a gap exists. First, if the number of non-zero neighbours, $m$, within a $3 \times 3$ window of an edge point is 1 or 2, a gap mav exist: if more than two non-zero points exist there is no gap. If $m=1$, a gap exists. If $m=2$, then calculate the absolute difference of their row, $D_{R}$, and column, $D_{C}$. numbers within the $3 \times 3$ window. A gap exists if $D_{C}$ and $D_{R} \neq 2$ and $D_{C}$ $+D_{R}<2$;

ii. When a gap exists, check the eight neighbours around the gap coordinates within the all-maxima array. If any of these are non-zero, select the largest one and insert it in the edge map array;

iii. If a gap exists and there are no candidates in the all-maxima array, search for the largest magnitude amongst the same eight neighbours in the allminima array then insert that point's magnitude in the edge map: and

iv. If a gap exists and no candidate exists amongst either the all-maxima and all-minima arrays, the gap is a hole which cannot be filled. 
Note that these gaps normally occur when there are large changes in the direction of the gradient as the accuracy of $\alpha$ is often very bad, ie at corners and branches. For this reason, all maxima (or alternatively all minima if necessary) are considered due to the unreliability of the angle comparisons at such points.

In order to illustrate Korn's edge detection technique, consider again the example image provided in figure 3-7 (original pixel values are provided in figure 3-9). The first step in the technique convolves each non-border pixel in the original image with each of the two masks $G_{\text {: }}$ and $G_{j}$. The results of these convolutions, denoted $n_{1}$ and $n_{2}$, are shown if figures 3-13 and 3-14 respectively. Each point within $n_{1}$ and $n_{2}$ next have an angle and amplitude caiculated as per equations 3.10 and 3.11 which are stored in separate arrays. Note that as a result of the convolution operation, a total of four pixels are lost from the original width and height of the image. The results of these calculations are shown in figures 3-15 and 3-16. Each amplitude point is then checked to determine whether it is a maximum in any of the four directions. Once again, edge points cannot be checked which results in an additional loss in width and height of two pixels, ie a one pixel border is lost. All resulting maxima have their direction checked against the angle $(\alpha)$ array. If the directions match, the amplitude value is stored in the edgepoint array. For example, the point at $i=3, j=3$ in figure $3-15$ has the value 151.472 and is a maxima in directions $S_{1}$ and $S_{4}$ (see figure 3-12). Its corresponding angle in the angle array is $252.5^{\circ}$. As the directions $S_{1}$ and $\alpha$ match within $\pm 22.5^{\circ}$, the point is recorded as an edge point. Regardless of the outcome of the angle comparison, all maxima encountered have their values stored in the all-maxima array. Each point is also checked to determine if it is a minima. Each minima encountered, regardless of its direction, is stored in the all-minima array. The results of 


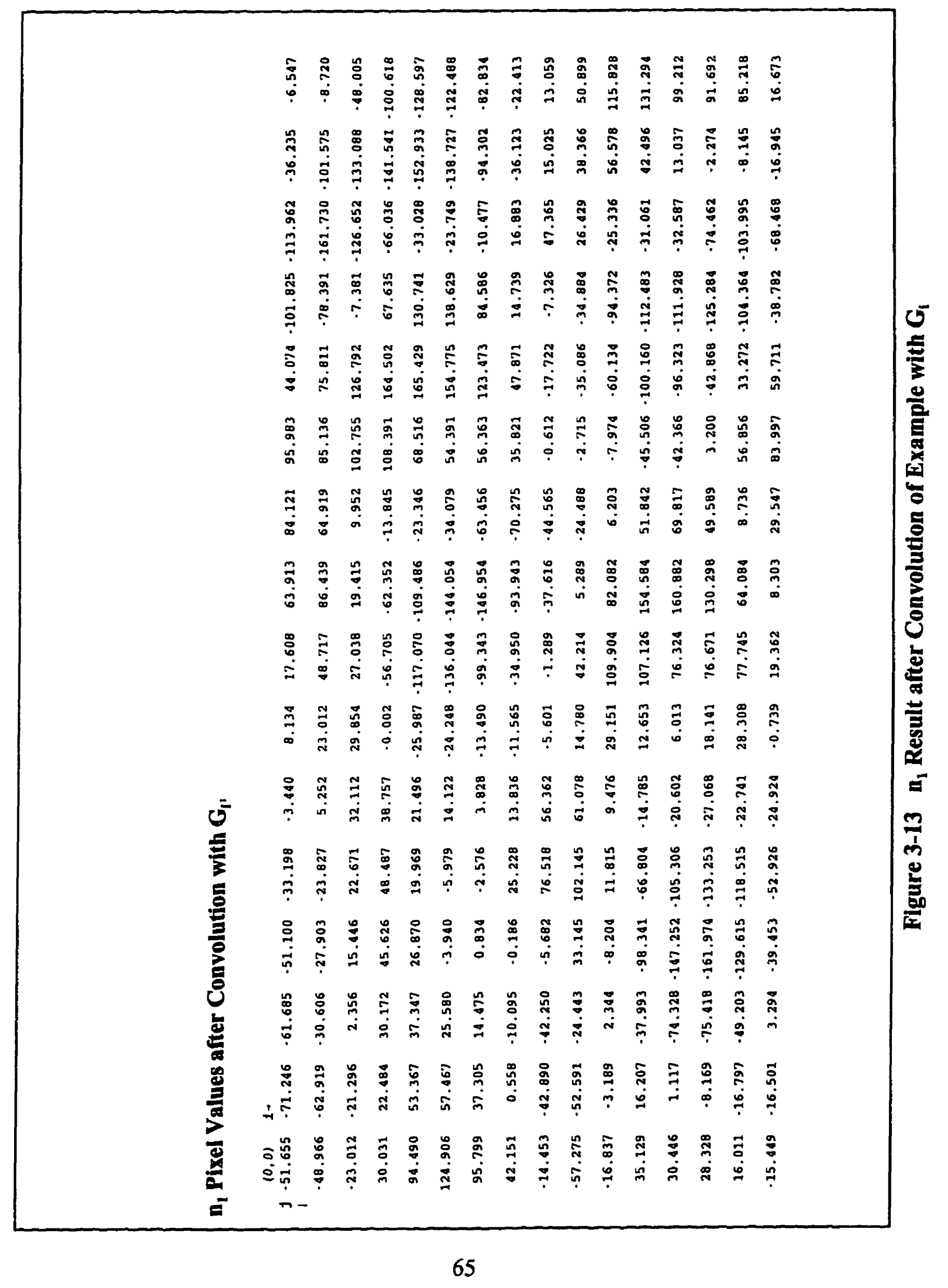




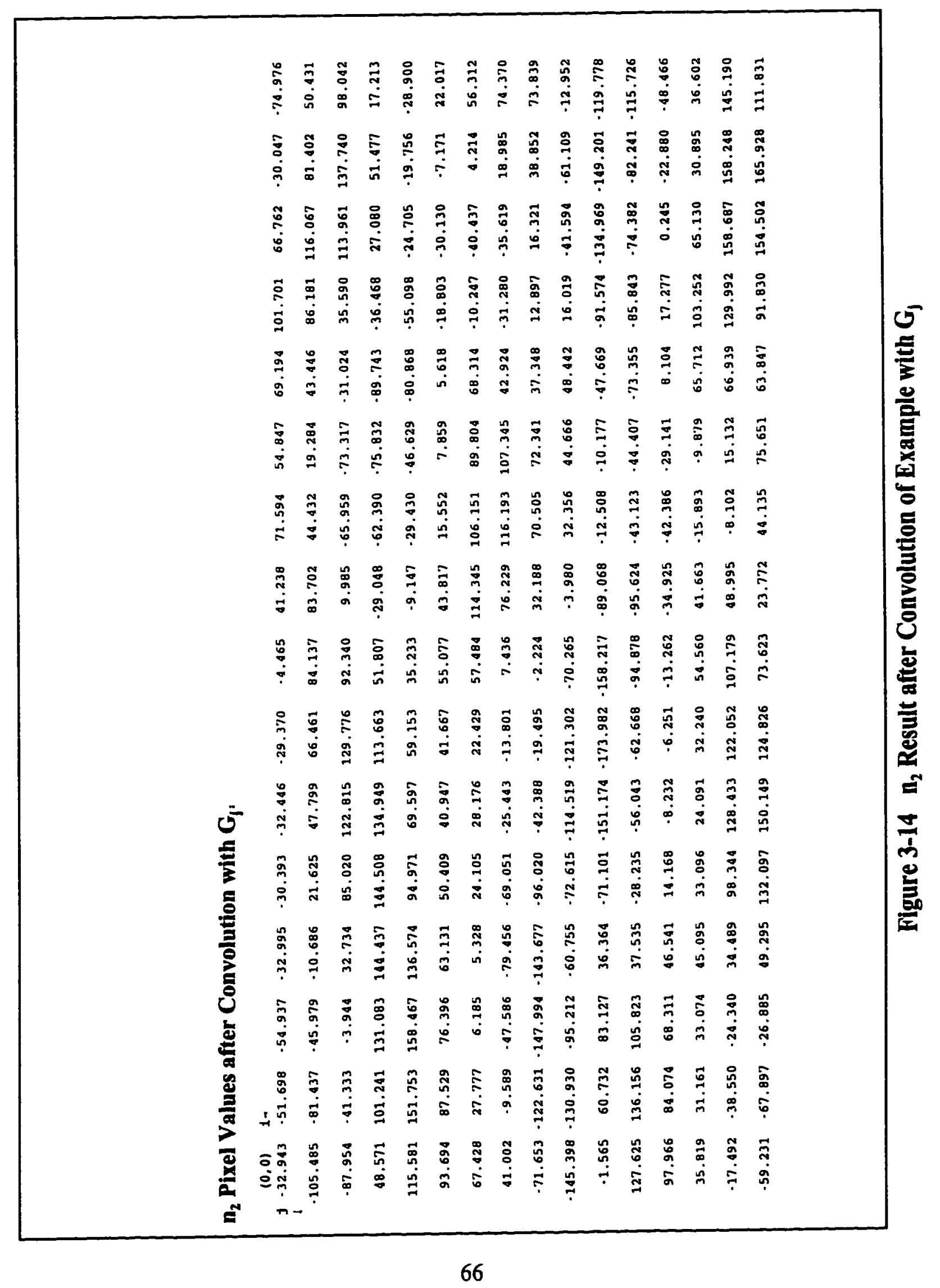




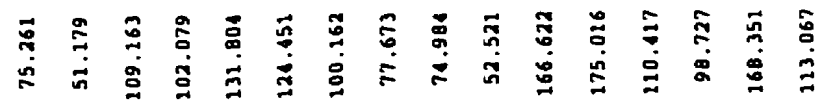

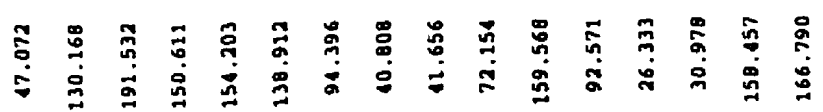

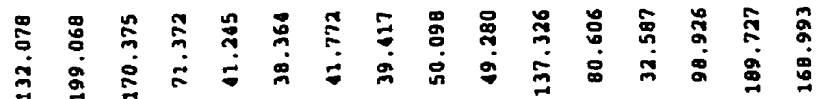

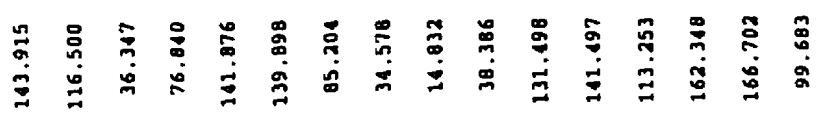

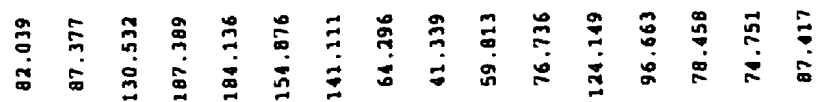

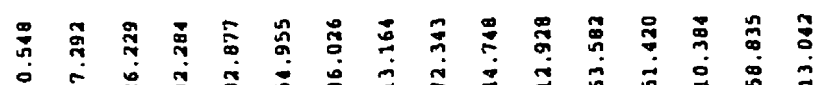

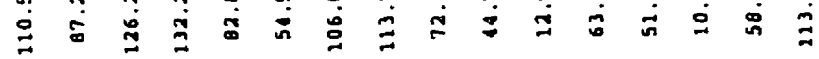

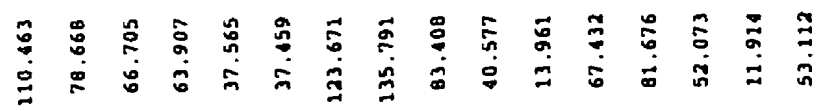

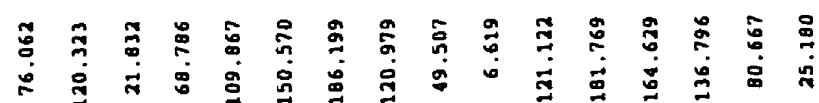

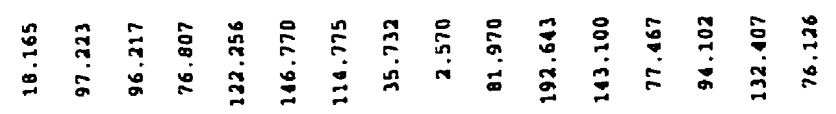

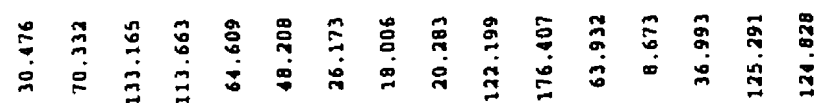

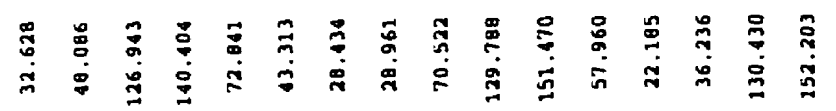

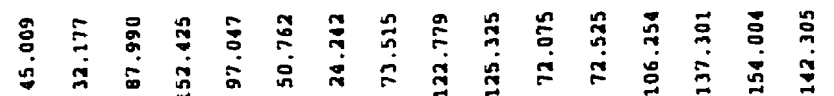

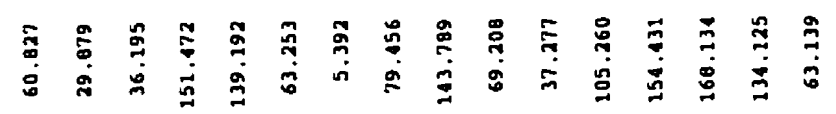

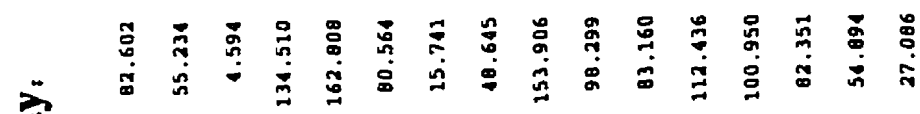

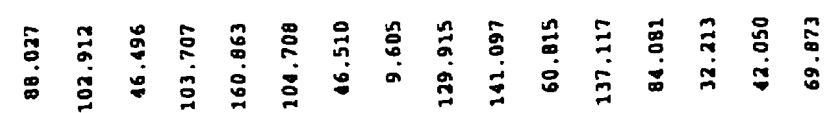

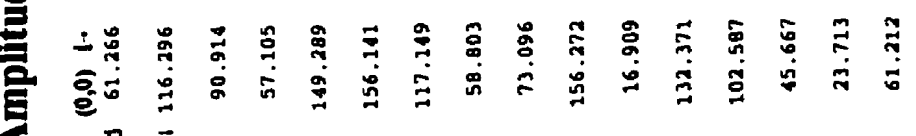




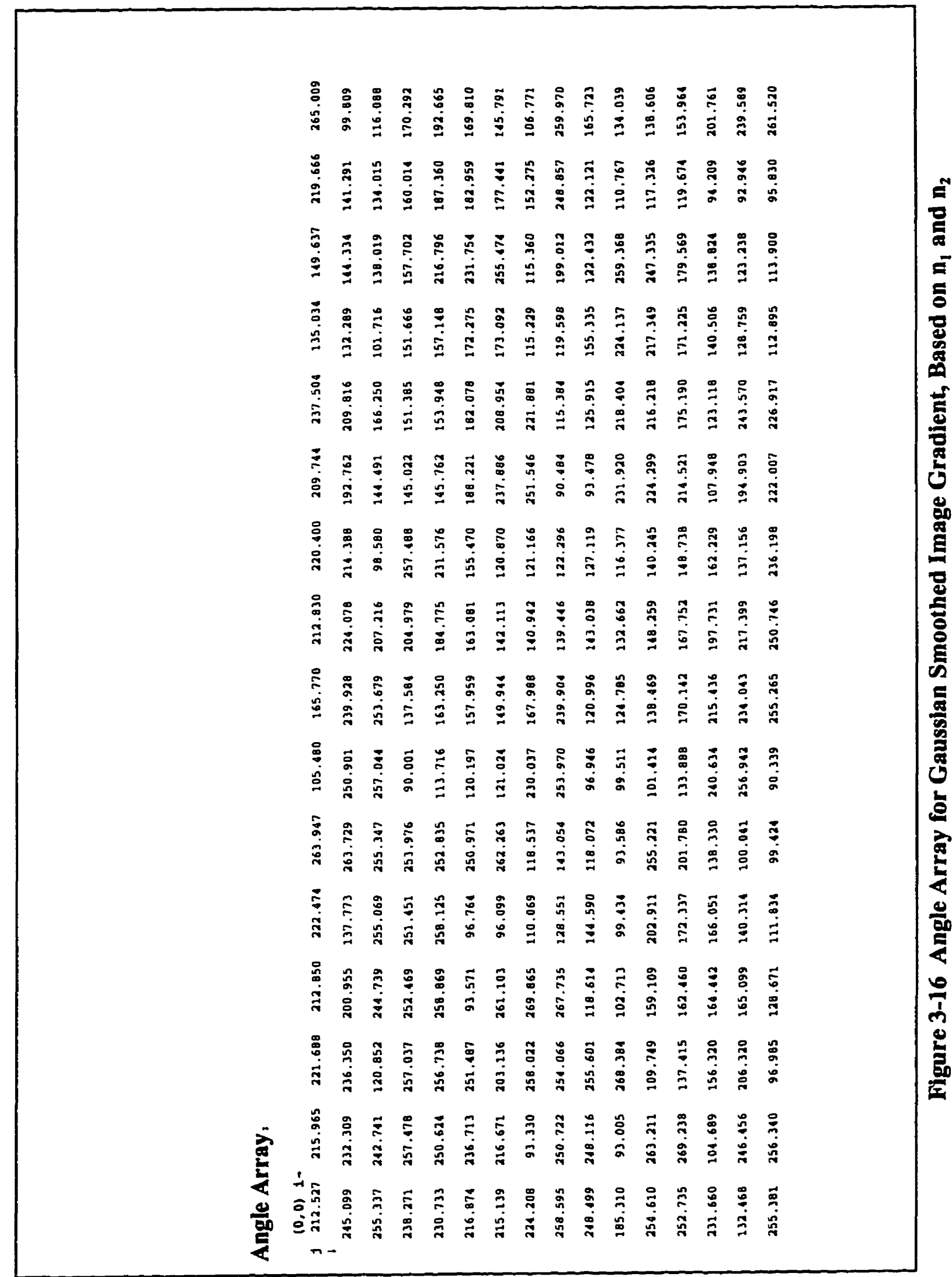




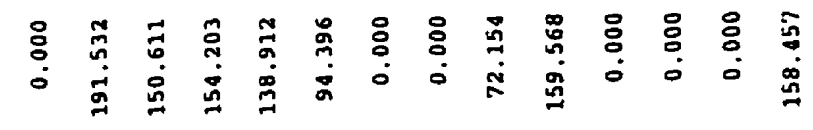

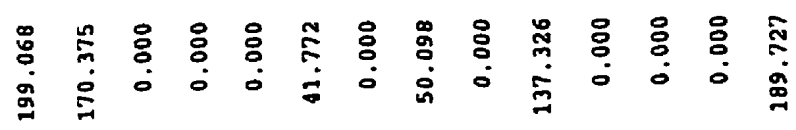

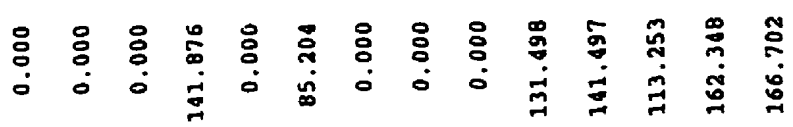

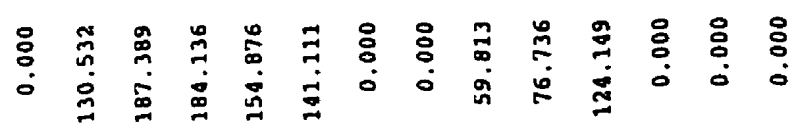

$$
\begin{aligned}
& \text { 芯 }
\end{aligned}
$$

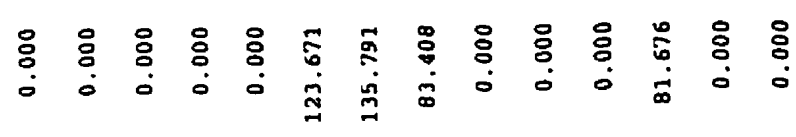

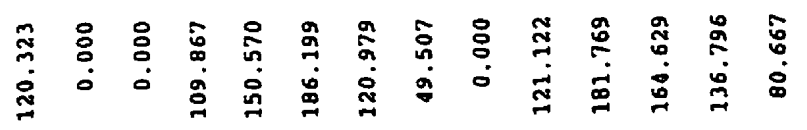

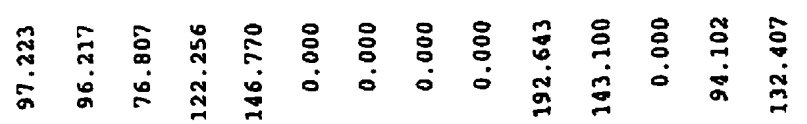

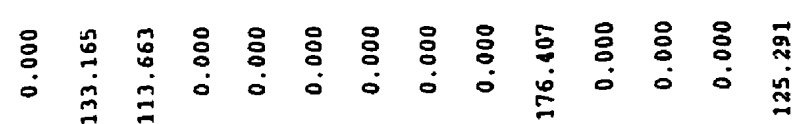

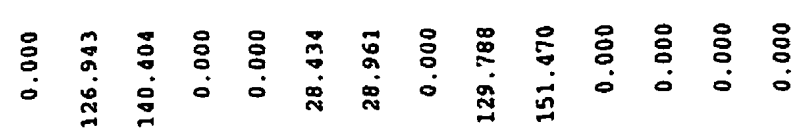

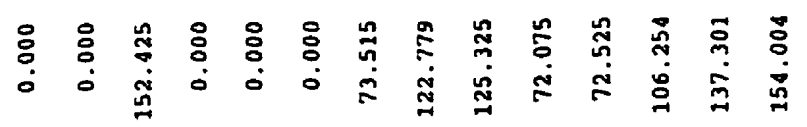

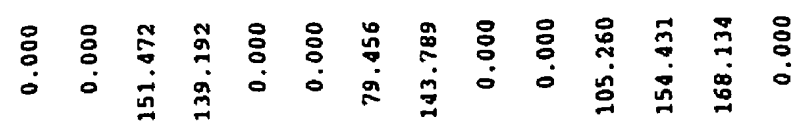

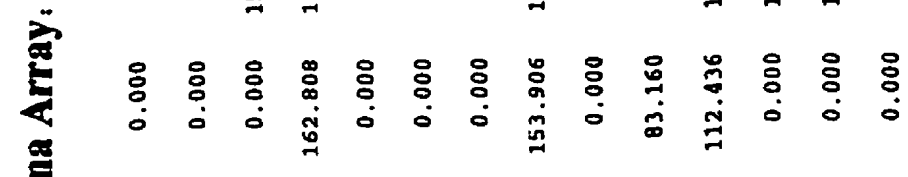

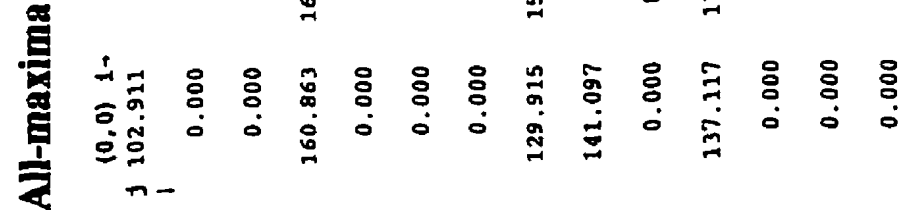




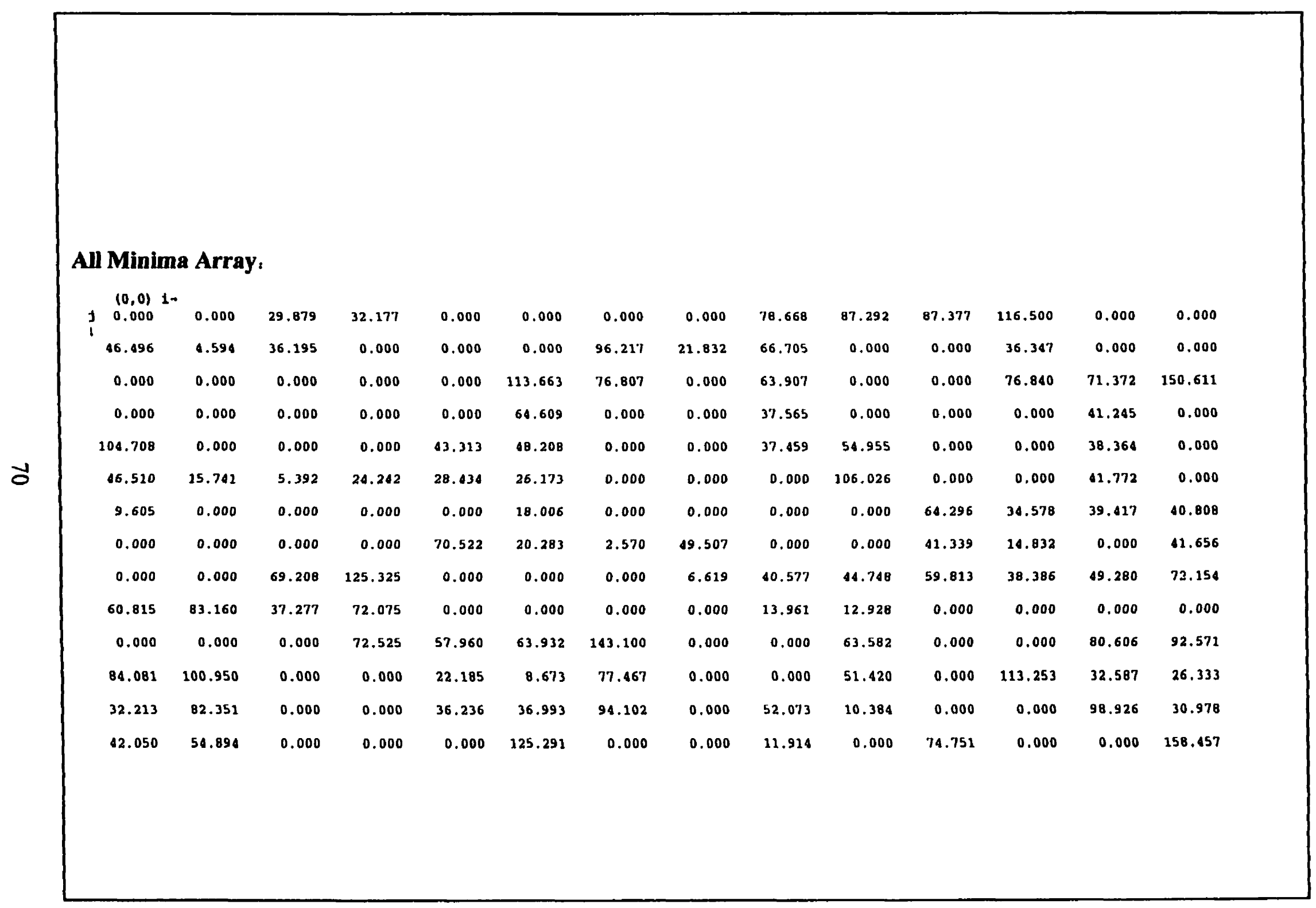

Figure 3-18 Array of All Minima Encountered 


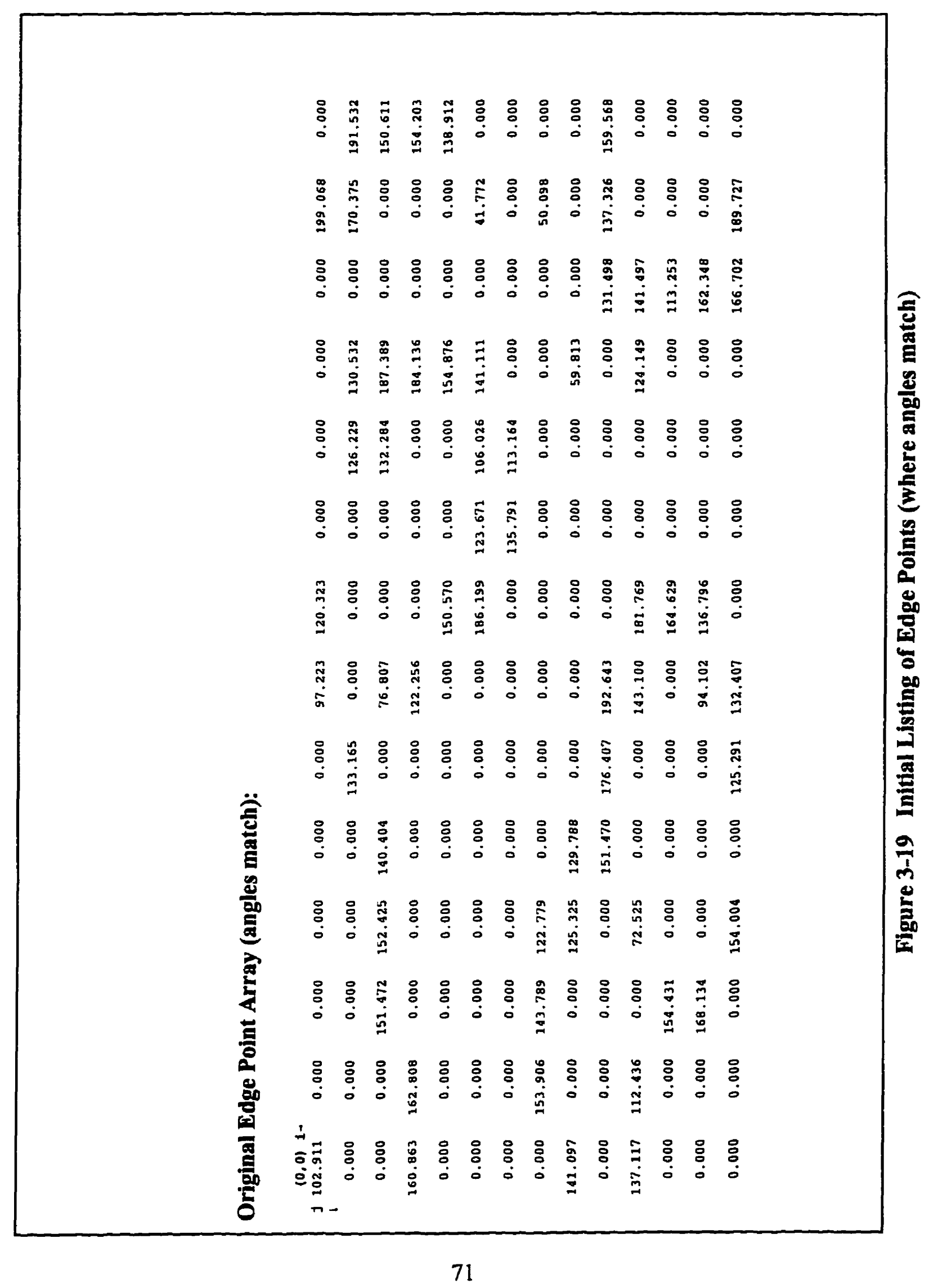


Edge Map after Gap Closing Technique was Applied:

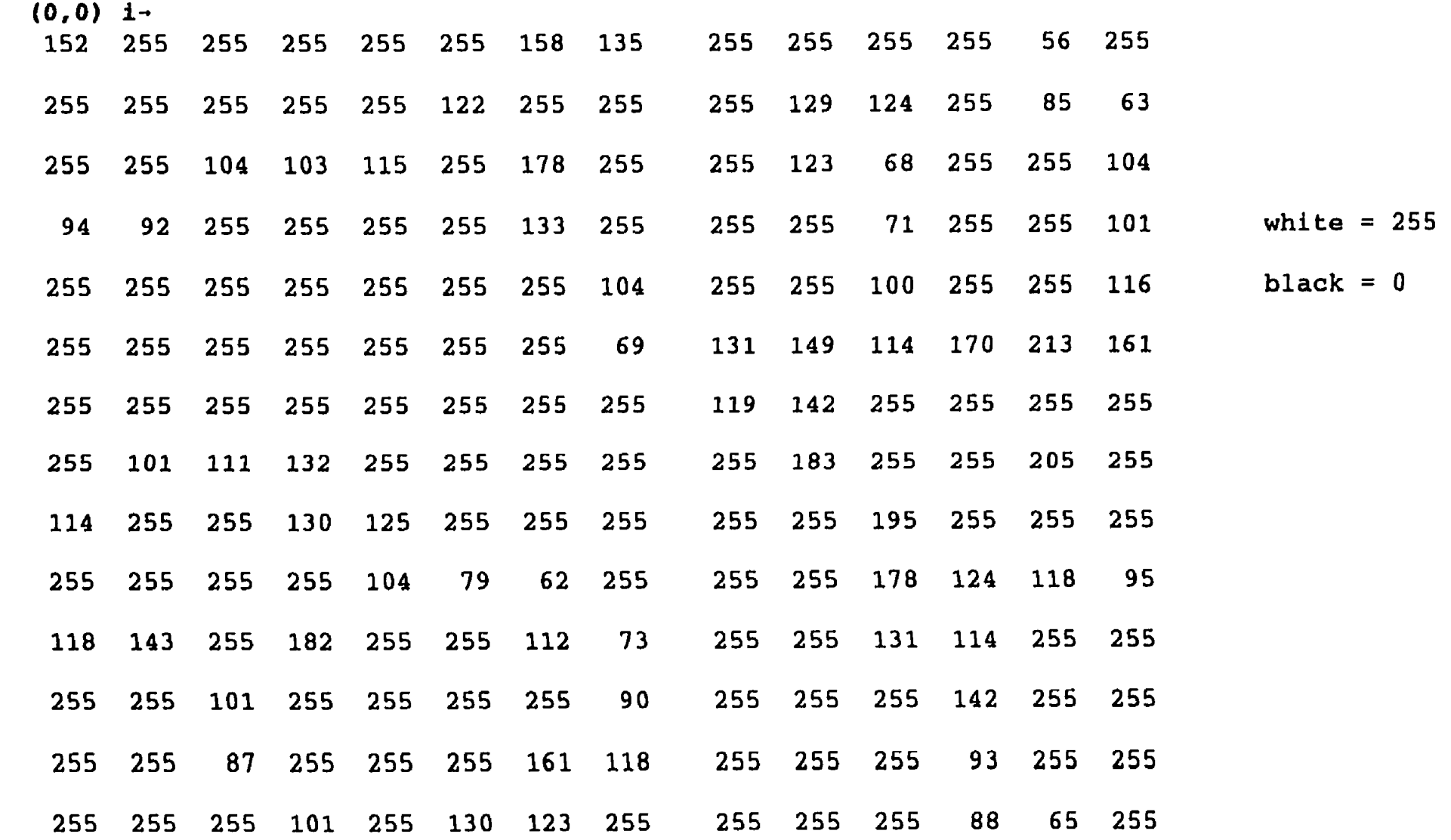

Figure 3-20 Final Edge Map after Gap Closing Efforts 
the all-maxima, all-minima and edge point search are displayed in figures 3-17, 3-18 and 3-19 respectively.

Once the edge points are determined, Korn's technique calls for a stage where the routine tries to fill all gaps encountered in chains of edge points using the all-maxima and all-minima arrays. As an example of this technique, consider the edge point a position (10,8) in figure 3-19. This point is a gap as it has only one neighbour. We then begin to attempt to close the gap by referring to the all-maxima array and checking around these coordinates for a suitable connector. Referring to figure $3-17$, the result of this is the value 76.731 at point $(10,9)$. This is then added to the edge point array and the array is continually checked until no more gaps exist which can be filled.

The end result of this "gap-closure" stage is shown in figure 3-20. This array of values constitutes the edge map derived for the subject image using Korn's DoG filter with $\sigma=0.8$. Note again that the photo_interpretation here is such that 255 is white and 0 is black.

Examples of results when this technique and the LoG technique are applied to M-mode Echocardiograms are provided and discussed in Chapter Five. Prior to presenting these findings, however, it is necessary to provide details on the application program which both produced these results and fulfills the second aim of this work - the derivation of a program to decode TIFF images, display these images, and apply the operations presented in Chapters Two and Three. 


\section{Chapter Four}

\section{Image Processing Program}

\section{4-1 Introductory Comments}

The first aim of this thesis was to study a range of image processing operations, the results of which have been presented in the previous two chapters. The next aim was to create a software package that was capable of importing, displaying, manipulating and storing digitized images. This effort has culminated in a program of some 9000 lines of code which is able to implement the various operations that have been discussed in this work.

This chapter begins with an explanation of the overall capabilities and limitations of the program. This is followed by a description of the processing environment that was created to act as an smooth interface between the user and the image processing capabilities of the program. The last section provides an overview of the main capabilities of the program and is broken down into four general sub-sections: image and workspace acquisition; image and workspace storage; image, workspace and file deletion; and, image processing.

Owing to the large scale and broad scope of the program, a number of conventions have been adopted to assist the reader with following the explanations provided throughout the chapter. They are designed to emphasize or clarify terms and names used by the program as well 
as assist the reader with following the flow of the program in response to user commands. To this end, they are listed below in two groups, programming conventions and command conventions, and each is followed by an example in square parenthesis:

Programming conventions:

1. Functions which have been written for the program have their names completely capitalized and are followed by parenthesis [IMPORT_IMAGE0];

2. Functions which are part of $\mathrm{C}++$ libraries are listed in lower case followed by parenthesis [toupper()];

3. Global variables and type specifications have their first letter capitalized [Active_image, Header_type];

4. Global constants are entirely capitalized [DISPLAY_AREA]; and

5. Local variables are named entirely in lowercase.

Command conventions:

1. The letter within a command used to select that command is printed in bold face [Import];

2. Primary commands are printed in italics [Delete];

3. Level two commands (menu options) are underlined and printed in italics [filters]; and

4. Level three commands (sub-menu options) are underlined and appear in regular typeface [Median filter].

Whenever possible, functions, global variables and global constants have been given names commensurate with their task within the program. For example, the counter which keeps track of the number of images that are currently in the workspace is titled "Number_of_images" and the function which creates a menu of save options is called "SAVE_MENU(0." When appropriate, the actual titles for such objects are used throughout this chapter to further acquaint 
the reader with the coding of the program.

\section{4-2 Program Design and Listing}

As was explained in Chapter One, the application program is written in the $C$ programming language and must be run in a DOS environment as the graphics mode used is not supported in the Windows environment. In our case, Borland $\mathrm{C}++$ version 3.1 was used to compile the program in order to meet these requirements. As a result of the top-down planning, the program is broken down into approximately 70 functions, most of which are independently compiled as part of the project. In a few cases this was not possible and these functions were linked to the main program for compilation. A copy of the documented program will be kept as a research report in the Math and Computer Science Department at the Royal Military College of Canada. The program listing begins with the main function followed by:

1. a header file which provides a list of global declarations;

2. a header file which provides global and library information for each of the files which could be independently compiled as part of the project;

3. a header file containing links to the functions which we were not able to independently compile as part of the project;

4. a header file listing prototypes for each of the functions;

5. a header file containing links to the $\mathrm{C}$ libraries that are used; and

6. all of the remaining functions, listed alphabetically.

At the beginning of each function is a "comment" block which provides: the role of the function, a list of arguments that it requires, the command path (if it is a one of the user commands), the algorithm it uses to fulfil its role, and an explanation of any values it returns on completion. 
Comments are then provided throughout the function to assist the reader in following the individual commands which comprise it.

\section{4-3 Processing Environment}

The program uses Borland C++ v3.1's graphics mode to display images, menus and text on the screen. It will work with CGA, EGA or VGA graphics cards. C++ library routines designed for use within the graphics mode are used to display lines, text, and pixel information for images. A complete list of such routines is contained in the Borland $\mathrm{C}++$ v3.1 Library reference pages 617 and 618 [Bor192-1]. All input and output is done using routines listed on pages 618 and 619 . All displays are in 16 gray scales only, as this is the largest number of gray scales that the compiler supports in the VGA mode. Nonetheless, all image pixel information is done in 256 gray scales as the mathematical operations can be executed more precisely at this resolution. Further, as many of the more sophisticated image processing applications and printers available are capable of displaying more than 16 gray levels, the output from operations can be stored in TIFF format and viewed at finer detail using such devices.

The processing environment created by the program is designed to produce a simple yet concise interface between the user and the program. It is intended to allow the user to select commands and follow the sequence of actions undertaken in response to these commands quickly as easily. Further, it is designed to provide as much information as possible to the user in icon, image or text format. This is accomplished using three techniques:

- dividing the screen into three sections to allow the user to manage images being used, 
- having a simple command set incorporating menus whenever possible, and

- displaying images with text information which relates their sequence within the program and background information.

The first technique involves the division of the workspace (screen) into three areas: command, display and bin. Figure 4-1 provides a pictorial representation of this divisioning. The COMMAND_AREA is comprised of a thin strip on the bottom of the screen designed to prompt the user for one of the primary commands. It is also used to provide some feedback to the user during the execution of some commands in the form of a text message.

The next section is the DISPLAY_AREA which makes up the majority of the workspace.

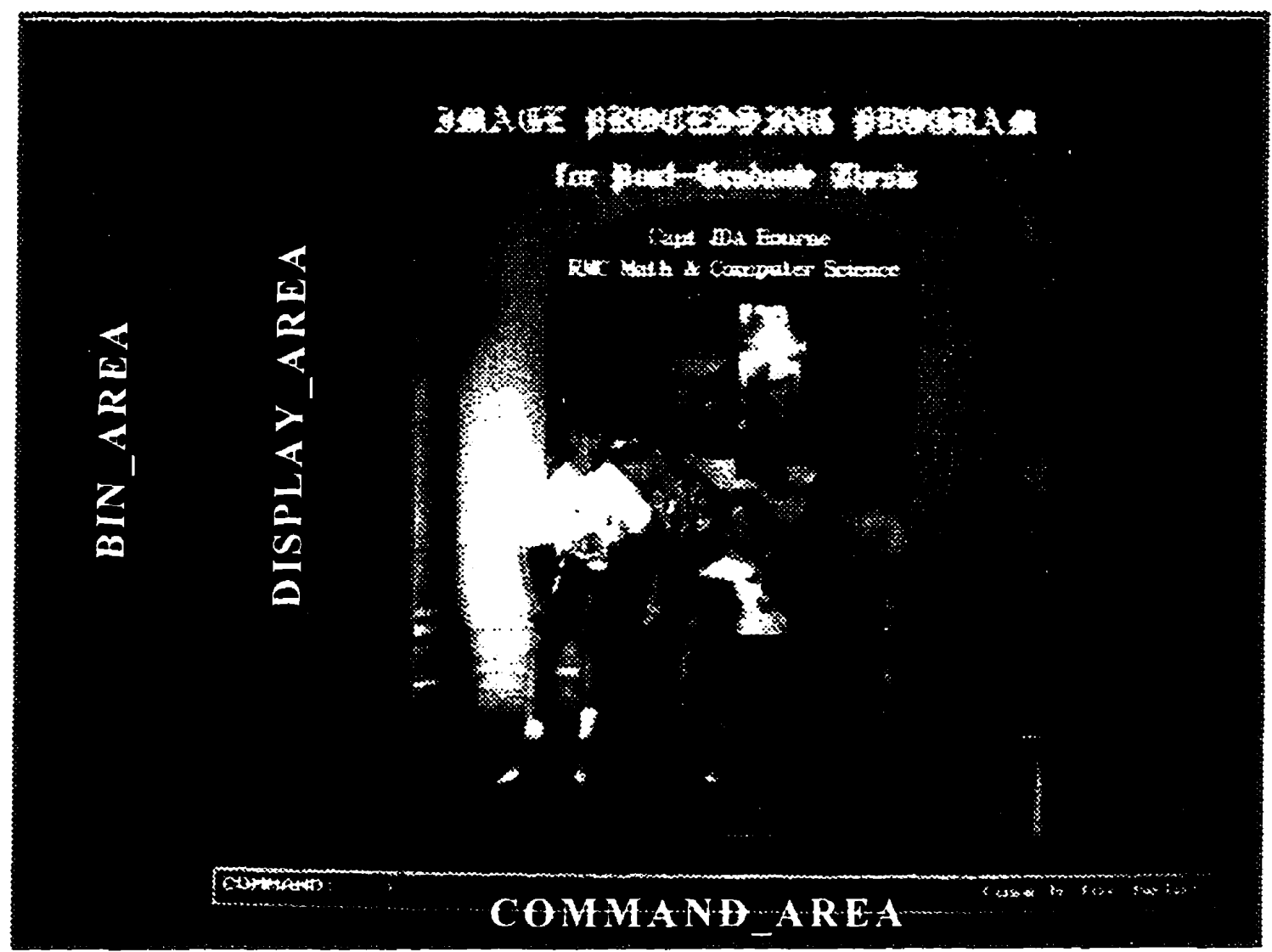

Figure 4-1 Program Workspace 
In this area, either one or two images will be displayed at the option of the user. The global variable Display_number records this value. The program begins assuming that two images will be displayed, although the user can change this using the options command. A maximum of two images was chosen because, as was explained in Chapter Two, every image process involves a maximum of two images. The option to display only one image in the display area was included to allow better viewing of large images. The final section is the BIN_AREA which is located on the left hand side of the workspace. In this area, up to 11 images can be stored in an icon format for future use or reference by the user. Once this area and the display area are full, images must be deleted from the program; however, they may be saved to memory for retrieval and use later as necessary. A maximum of 11 images was selected for the bin as this allows icons to be large enough to be discernable and enough "stand-by" images to be of use for most applications.

The second interfacing technique involves a simple command set. The primary commands available to the user are entered by using the command window described above. Some of these commands lead to menus of command options which are displayed as a list within a new window placed in the display area. If further menus are required, these are displayed in the same fashion. For the primary commands, a list of commands available is obtained by entering help in the command area. All commands can be entered using only the first letter and, again for simplicity sake, in most cases commands can be entered in either upper or lower case. If more than one letter is entered by the user, the excess is merely ignored by the program. The commands which are available to the user are presented as a tree diagram in figure 4-2. The figure shows the command flow for a number of tasks including:

1. altering the processing environment (active, clear, options, renumber, quit), 
2. importing images,

3. saving images,

4. deleting images from both the processing environment and memory,

5. processing images using various unary and binary operations, and

6. creating special test images.

Each of these capabilities will be explained in later sections.

The general flow of the program works as follows. An image is imported from memory and displayed "full-size" in the centre of the workspace area - full size meaning that all of the image's pixel information is shown without scaling (even if this means that the image will only be partially visible when it is larger than the available display area). This image is referred to as the "Active_image." After a new image is imported or created (using some process), it becomes the Active_image and the original image becomes the "Previous_image." If the number of images to be displayed in the display area is not changed (using options) from the default value of two, the Active_image is always displayed on the right half of the display area and the Previous_image is displayed on the left. If the default value for Display_number had been changed from two to one, only the Active_image would be displayed, in the centre of the display area, and the Previous_image would be moved to the top of the bin. When a new image is created or imported, the flow from right to left continues. If the Display_number is two and there are three images to be displayed in the workspace, the new image becomes the 


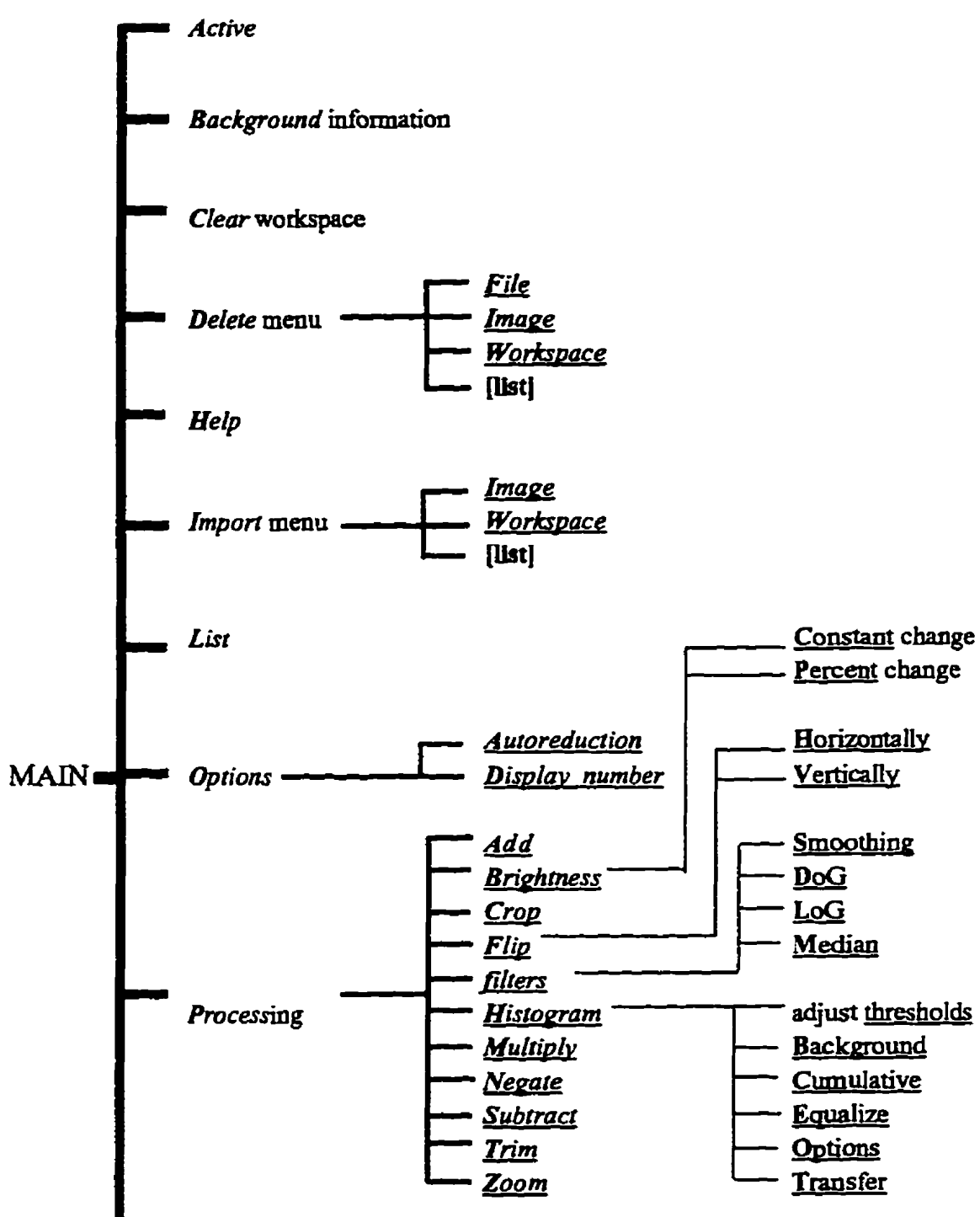

Renumber images

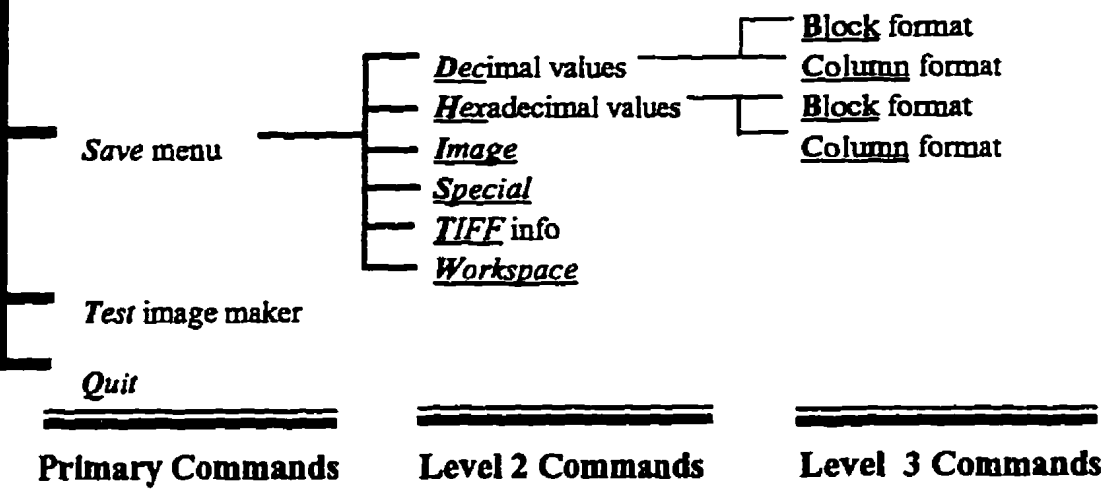

Figure 4-2 Image Processing Program Command Set 
Active_image, the original Active_image moves left and becomes the Previous_image, and the original Previous_image is moved to the bin. This process is summarized in figure 4-3. New images can be added until the total "Number_of_images" is 12: after this point the program will prompt the user to delete an image or images from the workspace. When it is full, there will either be two images in the display area and 10 images in the bin or one image in the display area and 11 images in the bin.

As each image is added, it is given an index number beginning with one. This number is displayed in the top left comer of the image window when an image is in the display area and at the top left corner of the icon if an image is in the bin (see figure 4-3). This is intended to assist the user in keeping track of the sequence of processed images. Each new image imported or created is assigned the next highest index number available. When an image is deleted, that index number is no longer valid and may not be used to reference an image during future processes. The maximum index number is 12 . Once this is reached, an image must be deleted if there are 12 images in the workspace or the images must be renumbered if some image(s) have already been deleted. This requirement has been incorporated solely for the purpose of simplifying the program code. It would be a simple task to automatically renumber the images whenever an image is deleted; however, this technique was not adopted as it would involve changing indexes without the user's direct involvement. We felt that it would be easier to keep track of which image had which index if a user was prompted before any changes took place.

As an additional "bookkeeping" feature, when an image is imported a text array is assigned to it called History[] which keeps track of the processes which are done on the image. An image's history is initialized with the image's name. As processes are done to it, 


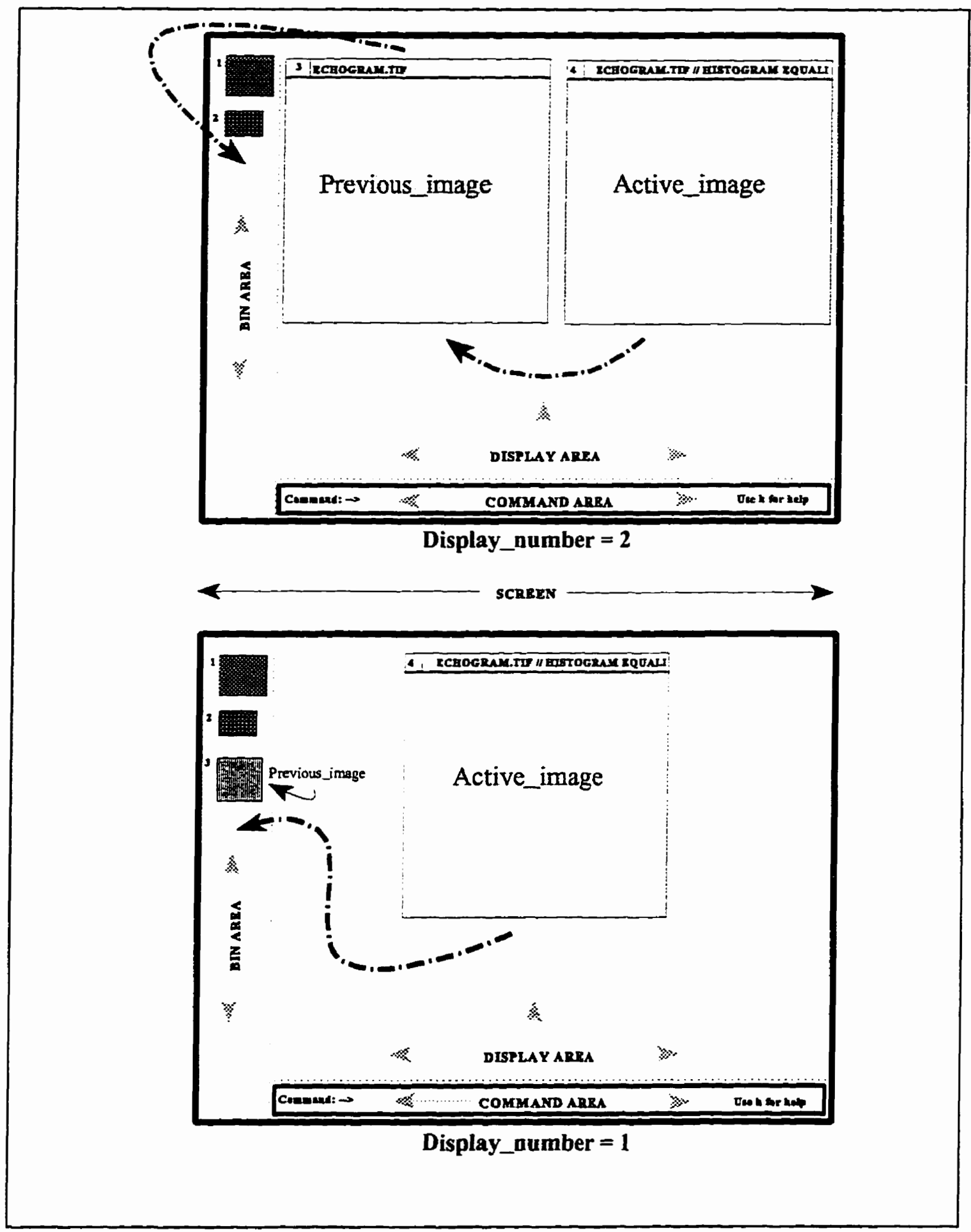

Figure 4-3 Image Display and Movement When One or Two Images are Displayed in the DISPLAY_AREA 
the details of the process are appended to the image's history. Different entries are separated by ' $/ /$ ' for ease of reading. For example, an image which has been histogram equalized may have a history like: "ECHOGRAM.TIF // HISTOGRAM EQUALIZED". When two images are added or subtracted, the new image's history is comprised of the histories of both images involved. separated by the appropriate operand. For example, an image created by the addition of two images may have a history: "[ECHOGRAM.TIF // HISTOGRAM EQUALIZED] + [ECHOGRM2.TIF // HISTOGRAM EQUALIZED]". It was intended that this feature would allow a user to recall the details of any previous processing that was done in order to reproduce the same effects again and/or recall which of several processes attempted was the most successful.

An image's history is displayed at the top of the image's window, to the right of the image's index, when it is in the display area. It is not displayed when the image is in the bin. Figure 4-3 also provides an example of index and history displays within a workspace. At any time, any existing image's complete history can be recalled using the background command. A text window is then created in the display area to provide this information to the user.

An image's TIFF information can be viewed at any time using the list command. This command generates an information window which will display the IFH and all IFD information for an image (byte by byte in hex). At the end of this listing of memory information, an option is provided whereby a user can view the image's tags in a decoded format - ie with the tag titles and meanings decoded into readable text.

Two final notes need to be added about the display of images in the display area. First, as an image's size can be well beyond its portion of the display area (either half or full), an 
additional feature was added to the program whereby a user may opt to have images within the display area scaled to fit the space available. This feature is called autoreduction and it is enabled through the options command. The default is that this feature is not enabled. If selected, it remains in effect until the user disables autoreduction within the options window. The intent of this feature is to allow the user to view the image or the effects of processing across the entire image if required. It is important to note that no data is lost when images are autoreduced. That is, their data files are not altered, only the display of their pixel information is compacted. When autoreduction is enabled and the program has had to reduce an image to fit it in the display area, the string " $<\mathrm{R}>$ " is displayed at the beginning of the history area of the image window to cue the user to this fact. See figure $4-4$ for an illustration of this format.

Secondly, when the workspace contains more than one image the user may opt to change the active image at any time using the active primary command. When this command is selected. the user is prompted for the index number of the new active image. When a valid response is received, this image is moved from its location to the active part of the display area. The image which was previously active is made the Previous_image and placed accordingly.

\section{4-4 Importing Images}

At this time, the program is only capable of importing images which are stored in TIFF format or in a temporary format which was designed for use by this program only. We decided early on to initially save images in a simple file format until a module was created to store 


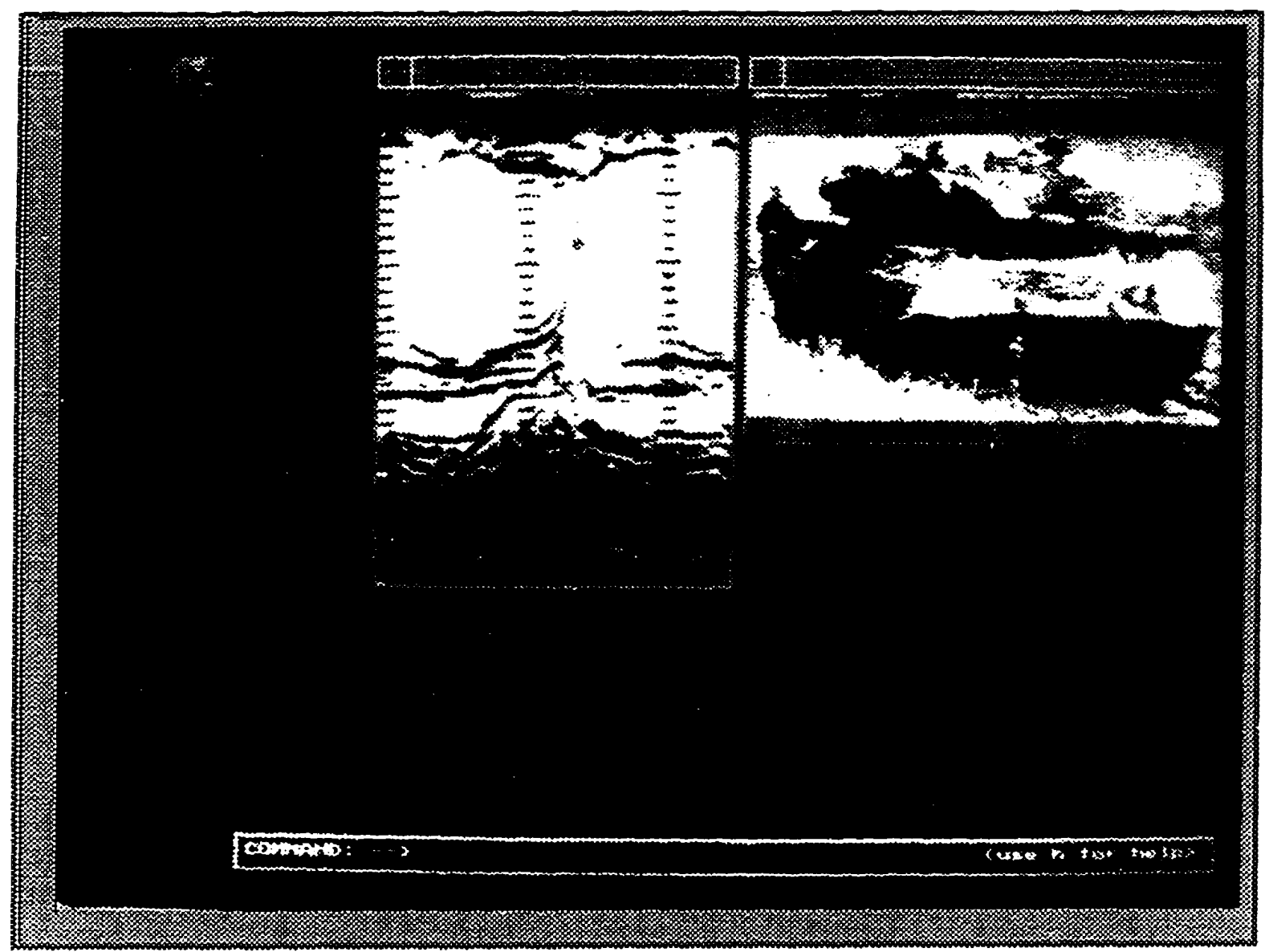

Figure 4-4 Example of Autoreduction

processed images in TIFF. At the time of writing, this final stage was not completed except for a special case which is described in the next section.

When a user selects import as a primary command, the main 0 function calls the IMPORT_IMAGEO function and a menu is presented listing the two import options available: image and workspace. If image is selected, the user is prompted for the name of the image to be imported. The default path is "C:LP-PROGUMAGES" and any image imported from outside of this path must have its path fully specified. The user may enter "[list]" to view files and directories in memory or on disk. When a name is entered, the program assumes that the file is a TIFF file unless the filename ends with the suffix ".SAV". This suffix is the convention for 
the temporary "image saving format" mentioned previously and explained in the next section. If the name is a valid one (the file exists), the IMPORT_MAGEO assigns the next index number to this new image.

If the image is a TIFF image, the IMPORT_MENUO function decodes the TIFF image using the functions GET_IFDO and DOWN_LOAD_DATA0. The actual tag information is retrieved by the function TAGSO which is called during the execution of DOWN_LOAD_DATA0. The TIFF information is used to set a number of global variables for the image such as: IFD[][], Width[], Height[], Bits_per_sample[], Photo_int[], X-resolution[] and so on. All of these global variables correspond to the critical tags discussed in Chapter Two. Finally, the image's name is stored at the beginning of its History[] string and program flow returns to main().

If the image is stored in .SAV format, for example "NEWIMAGE.SAV", the IMPORT_MENUO routine reads the pixel information from 'NEWIMAGE.SAV' and retrieves the critical tag information from a file called "NEWIMAGE.@@@”.

The other import menu option is "workspace". During program execution, a user can save an entire workspace for retrieval at a later time. The intention of this option was to allow a user to exit the program and save the work that had been accomplished to that point. The user could then resume processing where he/she left off by simply loading the saved workspace. When this option is selected, the MPORT_MENUO function calls IMPORT_WORKSPACEO which loads all of the workspace information from the file "FILENAME.WKS." All the referenced workspace's images are then imported from various image files stored in the same format as the .SAV convention, but with different standard suffixes which are described in the 
next section.

\section{4-5 Saving Images}

As mentioned in the previous section, a module for saving any image in TIFF format has not yet been written. Instead, images are saved in a standard format designed solely for use by this program as described below.

When the user selects the primary command save, the main 0 function calls SAVE_MENU() and a menu is displayed providing the user with the following options: $\underline{\text { Dec }}$ values, Hex values, Image, Special, TIFF information and Workspace. The role and operation of each of these sub-commands is discussed below.

Dec values: This option allows the user to save the pixel information, as decimal values, for an image to a text file. The SAVE_MENUO function calls the MAKE_MEMORYLIST_FLEEO function which then prompts the user for a name for the text file where the pixel information will be stored. The default directory is "C:UPPROGUMAGE_IN.FO"; if the file is to be stored in a different directory or drive the path must be fully specified. The user is then queried as to whether block or column format is to be used. The block format stores the pixel array in the same column and row fashion as the image is displayed. The column format saves the pixel information in one column, each row of which contains 32 bytes. The purpose of this option is to allow the user to view the pixel information for an image in decimal format. This is particularly useful when debugging new routines or when the user wishes to view the specific results of some operation on a pixel-by-pixel basis. 
Hex values: This option is identical to the Dec values option except that the pixel information is stored as hexadecimal values.

Image: In the absence of a TIFF writer module, this format was selected as a simple interim method for the storing of images by the program. The SAVE_MENUO function calls the SAVE_IMAGEO function which queries the user as to which image in the workspace is to be saved. If only one image is present, this step is omitted. Once a selection is made, the function prompts the user for a name for the save file. The default path is "C:UP-PROGIMAGES": again, any change must include a complete path specification. The save file will automatically end in ".SAV" regardless of any suffix provided by the user. Once a name has been provided, the function ensures that the file does not already exist; if it does, it queries the user as to whether the file is to be overwritten or a new file name is to be provided. The function then copies all of the images pixel information to this ".SAV" file and then calls the function MAKE_DATA_FILE0 to create a data file (same filename with ':@@@' suffix) containing all of the critical TIFF information for the image. The TIFF info is stored in a specific order and format which is originally specified in MAKE_DATA_FLEO. This order is crucial as the IMPORT_IMAGE() function expects a certain pattern when it tries to read a ".SAV" file. It should be noted that an image's History[] information is stored when this format is used.

Special: This is a recent addition to the program which constitutes a first draft of a TIFF writer module. It has a very limited ability to save images in a format which other TIFF readers (image processing programs, draw programs, text editors, printers and so forth) can decipher. Its operation is based on the idea of cloning. It begins when SAVE_MENU() calls the function SAVE_IN_TIF() which prompts the user for the image index and a filename for the file to be 
saved in the same manner as SAVE_IMAGEO. It then queries the user for the name of an existing TIFF file to act as a cloning source for the new file. This is very limited as the source TIFF image must be the same size or larger than the image to be saved. Additionally, the clone source must have its pixel information contained on only one strip. The function will copy all of the information from the clone source to the new file named by the user. It will then update all necessary TIFF tags to the image's settings and copy all of the image's pixel information to the save file. Note that the image's History[] is not saved when this option is used.

TIFF information: This option was created as part of the original TIFF reading module. Its purpose is to allow a user to save an image's TIFF information to a text file for future reference or text editing/printing. When the user selects this option, SAVE_MENU() calls the function SAVE_TIF_INFO() which prompts the user for an image index and a name for the target file for the text information. Once a valid filename has been received, the function calls the function TAGSO with a parameter which signals this function to print the TIFF information to the file named.

Workspace: This is the final save option available to the user. It provides the user with a means of saving the entire current workspace to memory for retrieval and further processing at some future time. When this option is selected, SAVE_MENU() calls the function SAVE_WORKSPACE0 which prompts the user for a reference name for the saved workspace. This is done in the same manner as Dec values except the default directory is "C:UPPROGIWKSPACE.SAV'. Once a valid filename has been received, the function automatically sets the suffix for the name provided to ".WKS" and saves all of the critical workspace variables to this file, such as Current_image, Previous_image, Next_image, Number_of_images and so 
forth. The function then saves each of the program's images to memory using suffixes which describe the type of the file:

.T\# - pixel file for image with index \# (where $1 \leq \# \leq 12$ );

.D\# - data file for image \# (which is the same as a.@@@ file);

.B\# - pixel file for bin icon of image \#;

$.1 \#$ - pixel file for reduced version of an image when autoreduction is "ON" and Display_number $=1$; and

.2\# - pixel file for reduced version of an image when autoreduction is "ON" and Display_number $=2$.

Note that the last two file types are only created if the autoreduction option is "ON".

\section{4-6 Deleting Images}

When a user is finished with an image in the workspace, or when the workspace is filled with 12 images, an image can be deleted using the primary command delete. Additionally, the delete command allows the user to delete a file from memory or a workspace from memory. When delete is selected, the main 0 function calls DELETE_MENUO to provide the user with a menu of these options.

If the user selects image from the menu, DELETE_MENU() calls DELETE_IMAGEO which then queries the user as to which image (if more than one exists) is to be deleted. If the choice is valid, the function confirms that the user wants to delete the image selected. When an image is deleted from the workspace, its index number ceases to be valid and the program's temporary files (TEMP\#, BIN\#, RED1-\#, RED2\#) for the image are deleted. All other images and indexes are not altered. A user can, of course, save the image to memory before deleting it 
from the workspace in case the image is required at some future time.

The next option is the deletion of a file from memory. This permits the user to do some basic file management from within the program. This would be useful, for example, if only limited space remained in memory and a user wanted to save a file or workspace. When the file option is selected, DELETE_MENU() calls DELETE_FILE() and the user is prompted for the name of a file to be deleted. The "[list]" option is again available for viewing the contents of different directories. Once a valid selection is made, the function deletes the file selected from memory.

The final option is the deletion of a workspace from memory. This can be done using the file option above; however, this could involve the individual deletion of up to 47 files. To simplify this task, the workspace option sets the current path to the default directory for saved workspaces (C:UP-PROGIWKSPACE.SAV) and takes care of all files related to a particular workspace. When the workspace option is selected, DELETE_MENUO calls DELETE_WORKSPACE() which begins by prompting the user for the reference name of the workspace to be deleted. The "[list]" option is again available. Once a valid name has been selected, the user's intent is confirmed. The function then deletes all files relating to the workspace selected from memory.

\section{4-7 Image Processing Operations}

The various operations described in Chapter Two and Three are all available within the

program for processing images. To process an image, the user must select the primary command process which causes the main 0 function to call PROCESS_MENUO. A menu is then produced 
and displayed in the DISPLAY_AREA containing the following options: $\underline{A d d}$ Brightness Crop Flip, Histogram, Multiply, Negate Subtract. Trim Zoom and filters. The filters option leads to another menu containing: Median, Smoothing, LoG, and DoG. A brief explanation of all of these options is provided below. A more detailed explanation of the algorithm used for each operation can be found within the text block at the beginning of the appropriate function in the program listing, or within Chapter Two and Three.

In each case, the algorithm for the operation is modelled on the sequence:

1. Query user for any operation-specific information required:

2. Create a new image with the next available index number.

3. Make this the new Active_image and move the Previous_image and old Active_image to the left as appropriate:

4. Fill the new image's data file with values derived as a result of the operation being implemented;

5. Adjust the new image pixel values as required (normalization or thresholding); and

6. Copy the subject image's history to the new image's history and append a string describing the operation just implemented.

\section{4-7.1 $\underline{\text { Add }}$}

This binary operation adds the Active_image to the Previous_image. As such, if only one image exists an error message results. The user can set up the images to be added using the primary command active. When add is selected, PROCESS_MENUO calls the function ADDO which first checks whether the two images are the same size. If they are not, an error message results. The images are then added together as per the sequence listed previously and the 
History[] string is set up as: "[original Previous_image's history] + [original Active_image's history]". Note that if the two subject images have a different Photo_int, the function must take this into account and convert one of the two to the other's format. In the program, $A D D()$ uses the Photo_int of the initial Active_image as the basis for the addition and the Photo_int of the new image. A user can always adjust this after the addition process by using the negate option described below. Finally, when the pixel values are added together, the resulting pixels will fall in a range of $0 \rightarrow 510$ necessitating a normalization of the results in the fashion described in Chapter Two before being stored to the new image's data file.

\section{4-7.2 Brightness}

This unary operation allows the user to adjust the brightness of the Active_image using either a constant change or a percentage change. When this operation is selected. PROCESS_MENU() calls BRIGHTNESSO which first queries the user as to which type of brightness adjustment is required. Once a valid selection is made, the user is prompted as to whether the image is to be brightened or darkened.

If addition/subtraction of a constant is selected, the function queries the user for the constant value. This value is then added or subtracted from the image as appropriate. Note that the Photo_int of the image is very important. If the Photo_int is 0 , adding a constant value to each pixel will darken the image. If the Photo_int is 1 , the constant value must be subtracted from the image's pixels to darken it. The convention adopted for pixels which have values outside of $0 \rightarrow 255$ is that values above 255 are set to 255 and values below 0 are set to 0 . At the end, the History[] string of the new image is appended with the string: "// BRIGHTENED BY 
\#” or "DARKENED BY \#”, where \# is the constant value.

A similar process occurs when percentage change is selected. In this case, each pixel is altered to the percentage called for by the user. For example, a $5 \%$ increase in brightness could be obtained by multiplying each pixel by 1.05 when white is depicted as gray level 255 . The "BY \#" in the History[] string described previously is replaced by "BY \# \%".

\section{4-7.3 Crop}

This unary operation allows the user to select a particular section of the Active_image and create a new image comprised of only that section's pixels. This is useful if an image contains a great deal of redundant information or if a user wants to crop an image down to a certain size in order to add, subtract or multiply it with another image. When this option is selected, PROCESS_MENUO calls CROPO which begins by creating a cross-hair in the centre of the Active_image. This cross-hair is drawn using the XOR facility in Borland C+ + which sets the line colour (gray scale) to the complement of the pixel at the centre of the cross-hair. For example, the lines would be drawn at colour 50 if the centre pixel's value was 205 . Likewise, the lines would be drawn at a colour of 0 if the centre pixel was 255 . This has the effect of ensuring that the cross-hair is always in contrast to the pixels around it, making it readily visible. A window is also created in the top left corner of the screen showing the cross-hair's coordinates within the image.

The user can move the cross-hair within the limits of the image using the numbers 1 through 9. The movement of the cross-hair corresponds to the layout of the number keys on the right hand side of an IBM enhanced keyboard. Each number entered causes the cross-hair to 
move I pixel in the specified direction. This process can be sped up to jumps of five pixels by pressing the '+' key at any point. The cross-hair can then be slowed down to one pixel per entry by hitting the '-' key at any time. In this fashion, the user positions the cross-hair in one of the corners of the area to be cropped. Once in position, the user must hit the enter key to select that location as one of the corners.

At this point, a new cross-hair is produced which is moved like the first. The position window now displays the selected coordinate, the coordinates of the new cursor, and the width and height of a rectangle using these two points as opposing vertices. As the new cross-bair is moved, a rectangle is drawn using the current coordinates as opposing vertices. The sides of this rectangle are drawn in the same fashion as the cross-hairs. The user moves the new cross-hair until the area to be cropped lies entirely within the rectangle. At this point the enter key is hit a second time and the function queries the user as to whether the range specified is to be used. a new range is to be set, or no cropping is to be done. The user can refer to the position window to confirm the details of the crop before actually creating the new image. Once finished. the function follows the standard sequence to create the new image with an additional step " 2.2 " wherein it sets the new images Width and Height as per the coordinates of the rectangle. Once the cropped area's pixel information is transferred, the function copies the subject image's history to the new image's history and appends the string "// CROPPED $\left(x_{\text {min }}, y_{\min }\right)\left(x_{\text {max }}, v_{\text {max }}\right)$ ", where the two points are the coordinates of the upper left hand corner of the crop area and its opposing corner. 


\section{4-7.4 flip}

This operation takes an image and flips it either horizontally or vertically. In effect, a mirror image of the image is created in the chosen direction. For example, when horizontal is selected, the ith column of the image becomes the (M-1-i)th for an image of width $M$. The rows are not affected in this case. Similarly, when vertical is selected, the $i$ th row becomes the $(\mathrm{N}-1$ i)th row for an image of height $\mathrm{N}$ and the column orientation is not affected. The function executes in the standard sequence and appends the string "// FLIPPED $d$ " where $d$ takes the value ' $\mathrm{V}$ ' or ' $\mathrm{H}$ ' as selected by the user.

\section{4-7.5 Histogram Operations}

This unary operation allows the user to view and adjust the histogram of an image. This process is of use when a user wants to set thresholds for pixel values or improve the contrast within an image. When this option is selected, PROCESS_MENU() calls HISTOGRAM() which begins by creating a histogram window which encompasses almost the entire screen. In the upper $3 / 4$ of the screen a bar graph of all pixel values is displayed. The abscissa contains the values $0 \rightarrow 255$ which represents all possible gray values. The vertical axis is a scale from $0 \rightarrow$ max which acts as a scale for the number of pixels within the image having a particular gray value. The value "max" is the largest number of pixels counted for a particular gray value amongst all gray values. For each gray level, a vertical bar is drawn to scale indicating the number of pixels that contain that gray value. Below the abscissa, two vertical hash marks ('l") 


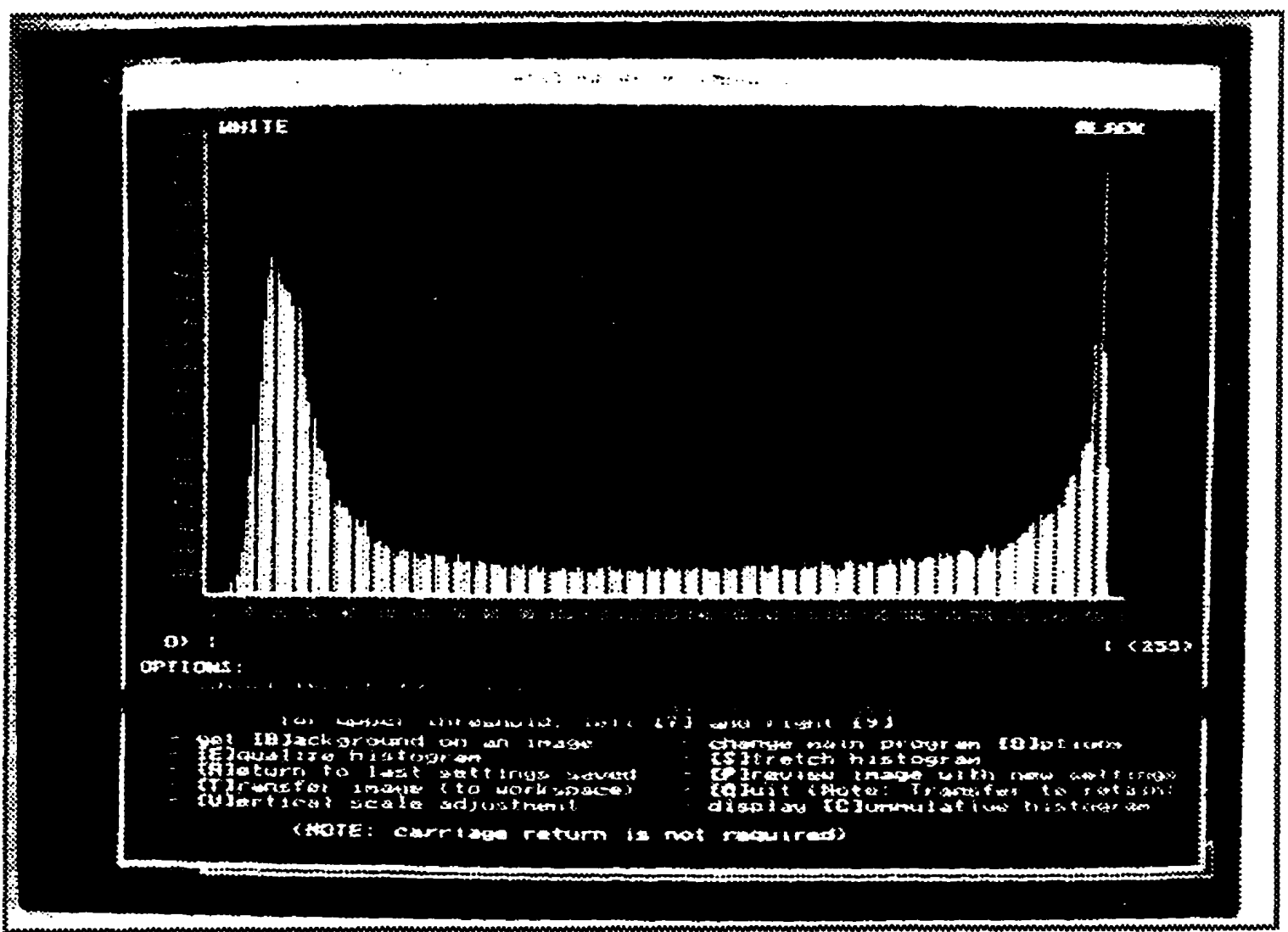

Figure 4-5 Example of Histogram Window

are displayed, one directly below the lowest pixel value encountered and the other directly below the highest pixel value encountered. These are used to adjust thresholds as described further on. To assist the user with keeping track of the Photo_int for the image, a text cue is placed above the 0 and 255 values to indicate whether the value represents a "WHITE" or "BLACK" pixel. Figure 4-5 provides an example of the layout of a typical histogram window.

At the bottom $1 / 4$ of the screen, a menu is displayed which provides the user with a number of options: adjust thresholds, provide background information on an image, display cumulative histogram, change program options, equalize the histogram, preview the image with the current threshold settings, return to last settings saved, transfer image with new settings to the workspace, adjust the vertical scale and quit the histogram module. These options are 
described below.

Adjusting thresholds: As described earlier, the hash marks under the abscissa mark the highest and lowest pixel values that are encountered. The user can adjust these thresholds by using the following number:

$\underline{4}$ - adjust lower threshold left,

$\underline{\mathbf{6}}$ - adjust lower threshold right,

I - adjust upper threshold left,

9 - adjust upper threshold right.

Note that the spatial orientation of these number keys on the right hand side of an enhanced IBM style keyboard conforms to their use as described above. Each time one of these keys is struck. the appropriate hash mark will move one pixel value to the left or right. The hash marks will not move outside of the range $0 \rightarrow 255$ nor will they cross one another or share the same pixel value. At any point, the user can view the resulting thresholded image using the preview option (described below). It should be emphasized that these settings are for experimentation and no new image will be created with these settings unless the user selects the transfer option before quitting the histogram module.

Background and Options: These two items operate exactly as per the primary commands background and options. The first is provided here to allow the user to review what work has been done on a subject image. The latter is provided to allow the user to adjust the view that will be provided when the preview option is selected.

Cumulative: This option allows the user to see a graph of the cumulative histogram. It is displayed in the same size as the original histogram and is written over the existing window. 
No actions are allowed from this window except returning to the original histogram window which is accomplished by striking any key.

Equalize: This option allows the user to equalize an image's histogram (see Chapter Two) to improve the contrast within the image. Once again, although the histogram displayed will be modified, a new image is not created with these settings unless the user opts to transfer the new image formed to the workspace. The user may, however, preview the image with the new settings.

Preview: This option allows the user to view what an image will look like under the current threshold and histogram settings. The image will be presented in the DISPLAY_AREA overtop of the histogram information. What is shown in the DISPLAY_AREA depends on the program options that are in effect. For example, if the Display_number is two, the image which acted as the source for the histogram (the Active_image at present) will be displayed on the left side of the DISPLAY_AREA and the preview image will be displayed on the right. If Display_number is set to one, only the preview image is displayed. Autoreduction operates as described previously. The options alternative allows the user to adjust these settings without leaving the histogram module. See Figure 4-6 for an example of the preview option.

Return: This option allows the user to reset the histogram and hash marks to the appropriate setting for the current Active_image. As such, all adjustment made using 


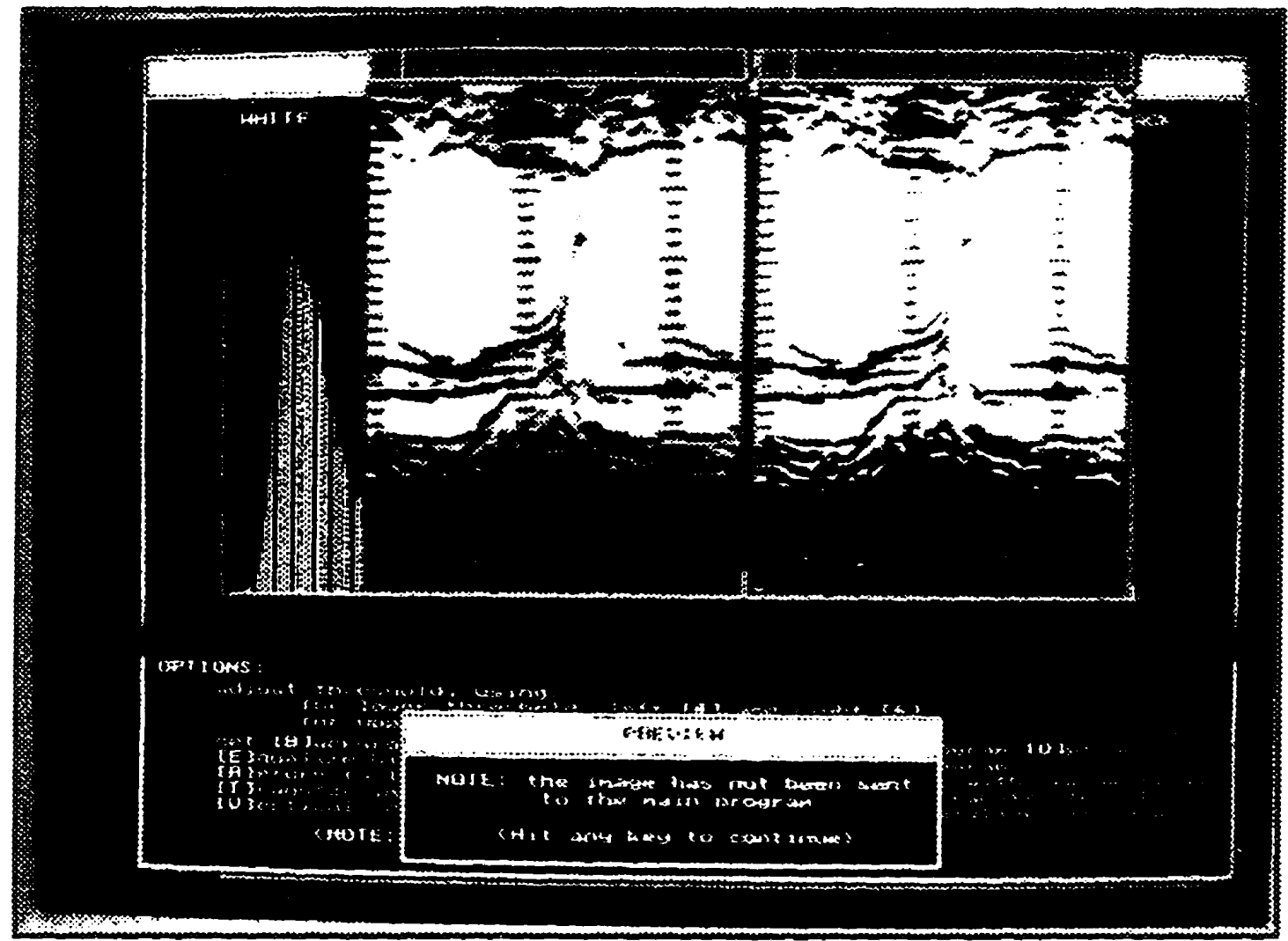

Figure 4-6 Example of Histogram Preview Option

thresholding or equalization without transferring will be removed. This allows the user to start histogram operations from the beginning without having to exit the histogram module.

Transfer: When the user selects this option, a new image is created in the workspace having the properties of the current histogram and threshold settings. The function does this according to the standard sequence using the current settings within the histogram window to adjust pixel values. The History[] string is appended with one of the following strings:
"HISTOGRAM THRESHOLDED ( $\min , \max )$ " where $\min$ is the lower threshold setting and $\max$ is the current upper threshold setting;

or

“HISTOGRAM EQUALIZED”. 
At the completion of this step the user is returned to the histogram window, which will contain the histogram of the new image. At this point, the user can continue to do histogram operations using the Active_image as a source or quit the module.

Quit: When this option is selected, the function simply returns control to the PROCESS_MENUO function. Once again, it should be emphasized that work which is not transferred to the workspace will be lost on exiting the histogram module.

Vertical: The final option available to the user is the ability to re-scale the vertical axis. Often certain gray values will cover a very large number of pixels. This can have the effect of creating a few relatively high "spikes" which the module will use as a basis for establishing the scale of the vertical axis. When the user opts to alter the vertical axis scale, the function prompts the user for a new max scale using the command window. The function then adjusts the vertical scale using this new max value. When lowered, gray values with relatively fewer pixel counts have their vertical bars raised, thus allowing the user to discern finer gray level details.

\section{4-7.6 Multiply}

This operation is identical in all ways to the add operation with the exception that the mathematical process done on the pixels in step 3 is multiplication instead of addition and the history reads as “[original Previous_image's history] X [original Active_image's history]”.

\section{4-7.7 Negate}

This unary operation allows the user to create a negative of an image. When this option is selected, PROCESS_MENUO calls NEGATEO which follows the standard operation 
sequence, filling the new image's pixels with the "negative" pixel values of the original Active_image. Recall that the negative of a pixel is " 255 - source pixel value." At the end of execution, the NEGATEO function adds the string "// NEGATED" to the end of the new image's History[] string.

\section{4-7.8 Subtract}

This operation is identical in all ways to the add operation with the exception that the mathematical process done on the pixels in step 3 is subtraction instead of addition and the history reads as “[original Previous_image's history] - [original Active_image's history]”.

\section{4-7.9 Trim}

This operation was included to allow a user to trim the edges of an image by an amount $\Delta \mathrm{i}$ from each side and $\Delta \mathrm{j}$ from the top and bottom. It is of particular use when adding images of different sizes such as is encountered when a filtered image is added or subtracted from its source image within this program. When the user selects this option, PROCESS_MENUO calls TRIMO which prompts the user for $\Delta \mathrm{i}$ and $\Delta \mathrm{j}$. The function then follows the standard sequence with an added step wherein it sets the new image's Width to the Width of the source image less $2 \Delta \mathrm{i}$ and the Height to the Height of the source less $2 \Delta \mathrm{j}$. The new image's History[] is then appended with the string "// TRIMMED $(\Delta \mathrm{i}, \Delta \mathrm{j})$ ". Note that this same operation could be done using crop, but this process would be more difficult and much slower. 


\section{4-7.10 Zoom}

The zoom option allows the user to scale the image to whatever scale is desired. When selected, PROCESS_MENU0 calls SCALE0 which begins by prompting the user for the scale factor. This value is entered as a decimal number, where expanding an image by $25 \%$ would require a scale of 1.25 for example. Once a scale is determined, the function follows the standard sequence with an added step wherein it sets the new image's Width to "Width[source image] $\mathrm{x}$ scale" and the Height to "Height[source image] x scale." The new image's History is appended with "// SCALED TO scale X".

\section{4-7.11 Filters}

The final processing option is filtering. When filter is selected, PROCESS_MENU() calls FILTER_MENUO which outputs a menu in the DISPLAY_AREA containing the filter

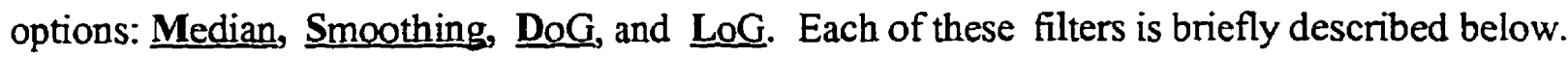
For a more comprehensive account of the details of the algorithms and the code which operates these filters, refer to the comments within each function provided in the program listing. Details on the theory behind each filter are contained in Chapters Two and Three.

4-7.11.1 Median: The median filtering routine determines the median value within a nine pixel mask for each pixel of a source image in the manner described in Chapter Two. As such, a one pixel border area is not processed and the output image is two pixels thinner and shorter than the source image. On completion, the output image's History[] string is appended with the string "MEDIAN FILTERED." 
4-7.11.2 Smoothing: The smoothing filter routine smooths an image using an averaging mask. It begins by prompting the user for the size (nxn) of the smoothing mask to be used: $3 \times 3,5 \times 5$ or $7 \times 7$. The routine then processes each non-border pixel by adding the $\mathrm{nxn}$ pixels within the mask and dividing by $\mathrm{n}^{2}$. As with the median filter routine, the smoothing filter's output will be two, four or six pixels narrower and shorter than the source depending on the size of the mask selected. On completion, the output image's History string is appended with the string "SMOOTHED $(\mathrm{n} \times \mathrm{n})$ " where $\mathrm{n}$ is 3,5 or 7 .

4-7.11.3 DoG: The DoG routine implements the DoG filter described in Chapter Three in order to detect edges within an image. The user is first queried as to how many passes the routine should make when trying to close the contours at the end of the process. Recall that the routine uses the files which record all maxima and all minima encountered within the amplitude file to close gaps after convolution with the DoG mask. This may allow the user to reduce the processing time, but with the possible consequent loss of some connecting edge points. The routine then prompts the user for the resolution required ( $\sigma$ value). Accepted values are $0.2,0.5$, 0.8 and 1.0. This value is used to calculate the convolutional masks $G_{i}$ and $G_{i}$. The routine then:

1. convolves the image with $\mathrm{G}_{\mathrm{i}}$ and $\mathrm{G}_{\mathrm{j}}$;

2. calculates the amplitude and angle values for each point;

3. finds all maxima and minima within the amplitude file;

4. records maxima, whose angle agrees with the angle file, in the initial edge map;

5. checks for gaps in the edge map as described in Chapter Three;

6. attempts to fill the gaps using values in the all-maxima file or all-minima file in 
that order: and

7. halts when all gaps that can be filled are filled or the maximum number of passes given by the user is reached.

When attempting to close contours, the routine informs the user of the number of the pass which is being conducted with a message in the COMMAND_AREA. When the routine finishes, the total number of holes which were not filled or could not be filled (if any) are provided to the user via a message displayed in the COMMAND_AREA. The routine will not continue from this point until a key is hit by the user. Once again, as with all convolution routines, the border pixels are ignored and the resulting image is smaller than the original by an amount which depends on the $\sigma$ value selected (as $\sigma$ is increased, more border pixels are lost). On completion, the new image's History[] string is appended with the message "DoG FLTERED (SIGMA = n)" where $\mathrm{n}$ is the $\sigma$ value selected by the user.

4-7.11.4 LoG: In the case of the LoG filter, the user is only prompted for a $\sigma$ value. Accepted values are $0<\sigma \leq 3$. This limitation was created for both filters in order to limit the size of the convolutional masks used within the program. The appropriate convolutional mask is then created (as described in Chapter Three) and the image is convolved with this mask. As an indication of progress, the routine informs the user as to which row is being processed with a message displayed in the COMMAND_AREA. During processing, border pixels are sacrificed in the same manner as for DoG filtering. On completion, the routine prompts the user for the type of output image that is required: one normalized in gray scale, a black and white mapping 
or an edge map. The user enters as many selections as required and the routine will create new images as instructed until a 0 is entered or the maximum image index is reached (12). For each image created, the History[] string is based only on the source image's and an appending message of either:

$$
\begin{aligned}
& \text { "LOG FILTERED - GRAY (SIGMA = n)" } \\
& \text { "LOG FLLTERED - B\&W (SIGMA = n)" or } \\
& \text { "LOG FILTERED - EDGE (SIGMA = n)" }
\end{aligned}
$$

as appropriate.

This ends the summary of the capabilities and limitations of the application program. In the next chapter, a number of the routines described above will be applied in order to enhance the edges in an image of an M-mode Echocardiogram. 


\section{Chapter Five}

\section{Experimentation and Results}

\subsection{Introductory Comments}

In the previous chapters, a large number of image processing operations were introduced and discussed at length. The application program is capable of over 40 actions of which approximately 20 are operations involved in the manipulation of pixel values within images. Rather than provide examples of the effects of all of these operations, this chapter will provide examples of the output of the two edge detection techniques presented in Chapter Three as well as some results of operations used in improving or comparing the various outputs such as histogram techniques, addition and subtraction.

As stated in Chapter Two, the primary purpose for developing this program has been to augment the research being done by Dr Shoucri regarding the enhancement and processing of Mmode Echocardiograms. As such, the test image selected is an M-mode image which has been digitized by a Hewlett Packard ScanJet IIc scanner. The original source image is provided in figure 5-I and its TIFF information is listed in figure 5-2. As the image is quite large (405x571), considerable computational time is involved (in some circumstances $>20$ minutes); however, a larger image was chosen in order to improve the clarity of the results and minimize relative losses 


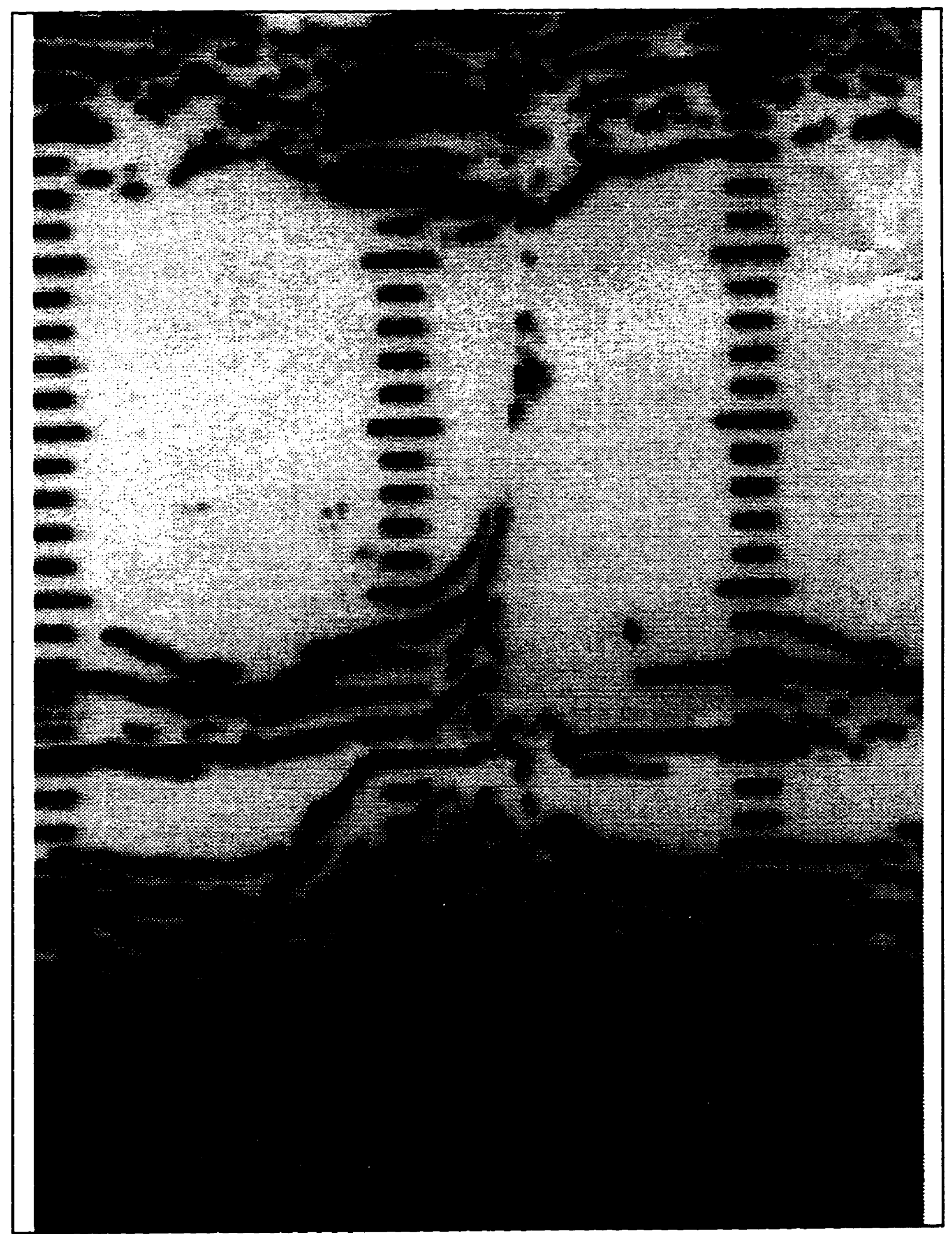

Figure 5-1 Test Image of M-mode Echocardiogram 
Detailed Tags Listing of primary IFD for image 1 with history $\rightarrow$ ECHO.TIF

$$
\begin{aligned}
& \text { Tag fe - new_subfile_type }=0(\mathrm{~h}) \\
& \text { Tag } 100-\text { width } \quad=195(\mathrm{~h})=405(\mathrm{~d}) \\
& \text { Tag } 101 \text { - height } \\
& \text { Tag } 102 \text { - bits_per_sample }=8(\mathrm{~h}) \\
& \text { Tag } 103 \text { - compression }=1(\mathrm{~h}) \\
& \text { Tag } 106 \text {-photo_int }=0(\mathrm{~h}) \\
& \text { Tag } 111 \text {-number_of_strips }=1(\mathrm{~h})=1(\mathrm{~d}) \\
& \text { Tag } 115 \text {-samples_per_pixel }=1(\mathrm{~h}) \\
& \text { Tag } 116 \text { - rows_per_strip } \quad=23 \mathrm{~b}(\mathrm{~h})=571(\mathrm{~d}) \\
& \text { Tag } 117 \text { - number of bytecounts }=1(\mathrm{~h})=1(\mathrm{~d}) \\
& \text { Tag } 11 \mathrm{a}-\mathrm{x} \_ \text {resolution } \quad=200.00(\mathrm{~d}) \\
& \text { Tag } 11 \mathrm{~b}-\text { y_resolution } \quad=200.00(\mathrm{~d}) \\
& \text { Tag } 122 \text { - gray response unit }=3(\mathrm{~h})(<->\text { thousandths }) \\
& \text { Tag } 123 \text { - gray response curve }=8(\mathrm{~h}) \\
& \text { Tag } 128-\text { resolution_unit }=2(\mathrm{~h})(<->\text { inches }) \\
& \text { Tag } 141 \text { - not recognized or new Tag ... }
\end{aligned}
$$


in positional accuracy as described in Chapter Three.

The Chapter is broken down into three sections. The first provides experimental results for processing the test image using the LoG filter. The next provides the same using a DoG filter and the final section is a discussion of the relative performance and merits of both approaches.

\subsection{Experimental Results Using the LoG Filter}

In this section, a "slide show" of results is presented illustrating the effects of LoG filtering on the test image. The test image is first processed using the LoG filter with a $\sigma$ value of 1.0. Figure 5-3 shows the result of this operation displayed in the normalized gray value format discussed in Section 3-4. This is followed by figure $5-4$ which shows the convolution results in a black and white mapping where pixels with negative values are displayed in white and pixels with positive values are displayed in black. The lines dividing these regions are the contours of the original image as detected by the LoG filter and a mapping of these contours is provided as figure 5-5. Figure 5-6 shows the result of adding the edge mapping in figure 5-5 to the original image in order to highlight the contours detected. In order to illustrate the effects of altering the resolution ( $\sigma$ ), figures 5-7 and 5-8 show the edge maps for the test image processed with $\sigma$ values of 2.0 and 3.0 respectively. 


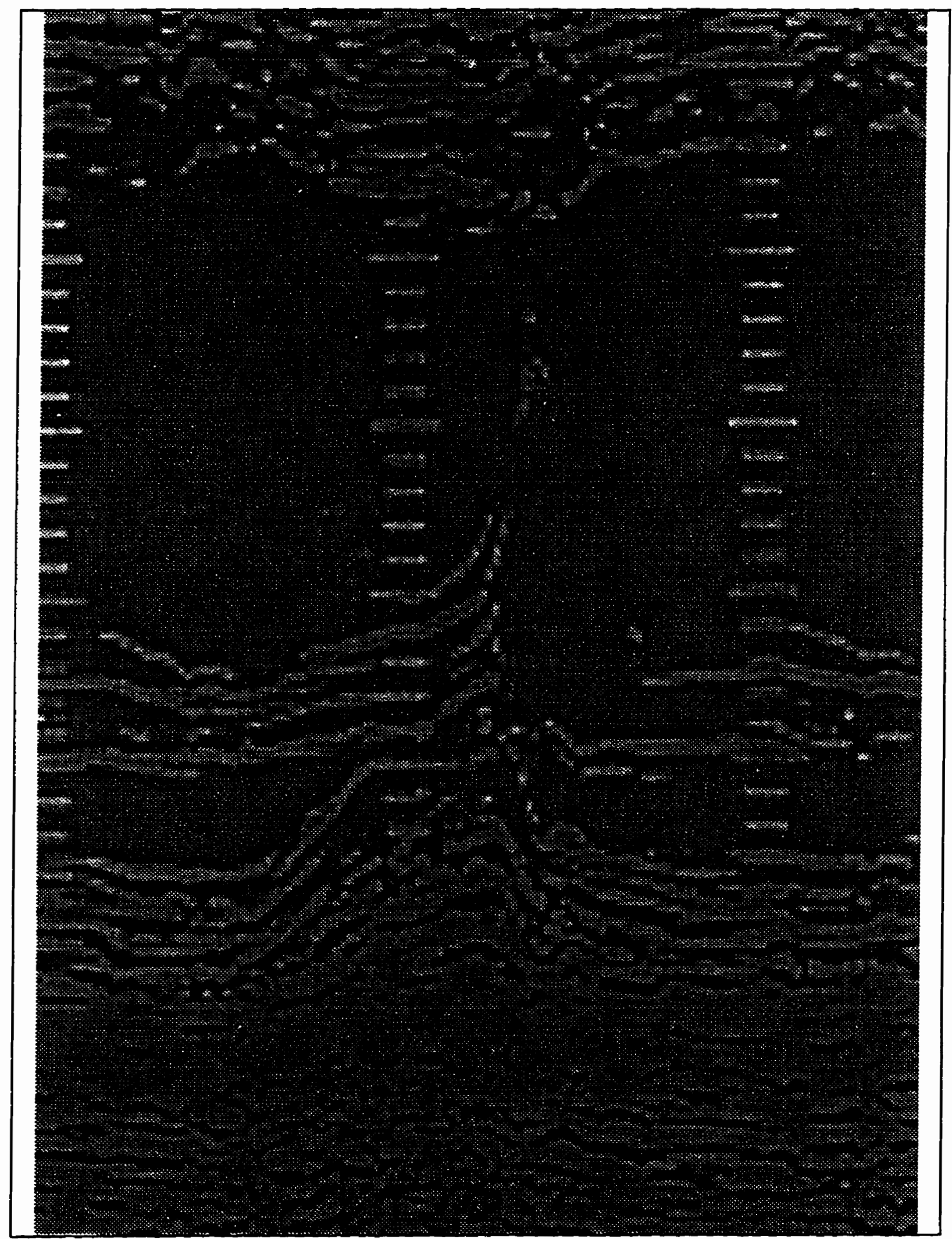

Figure 5-3 Normalized Gray Scale Result of LoG Filtering ( $\sigma=1.0)$ 


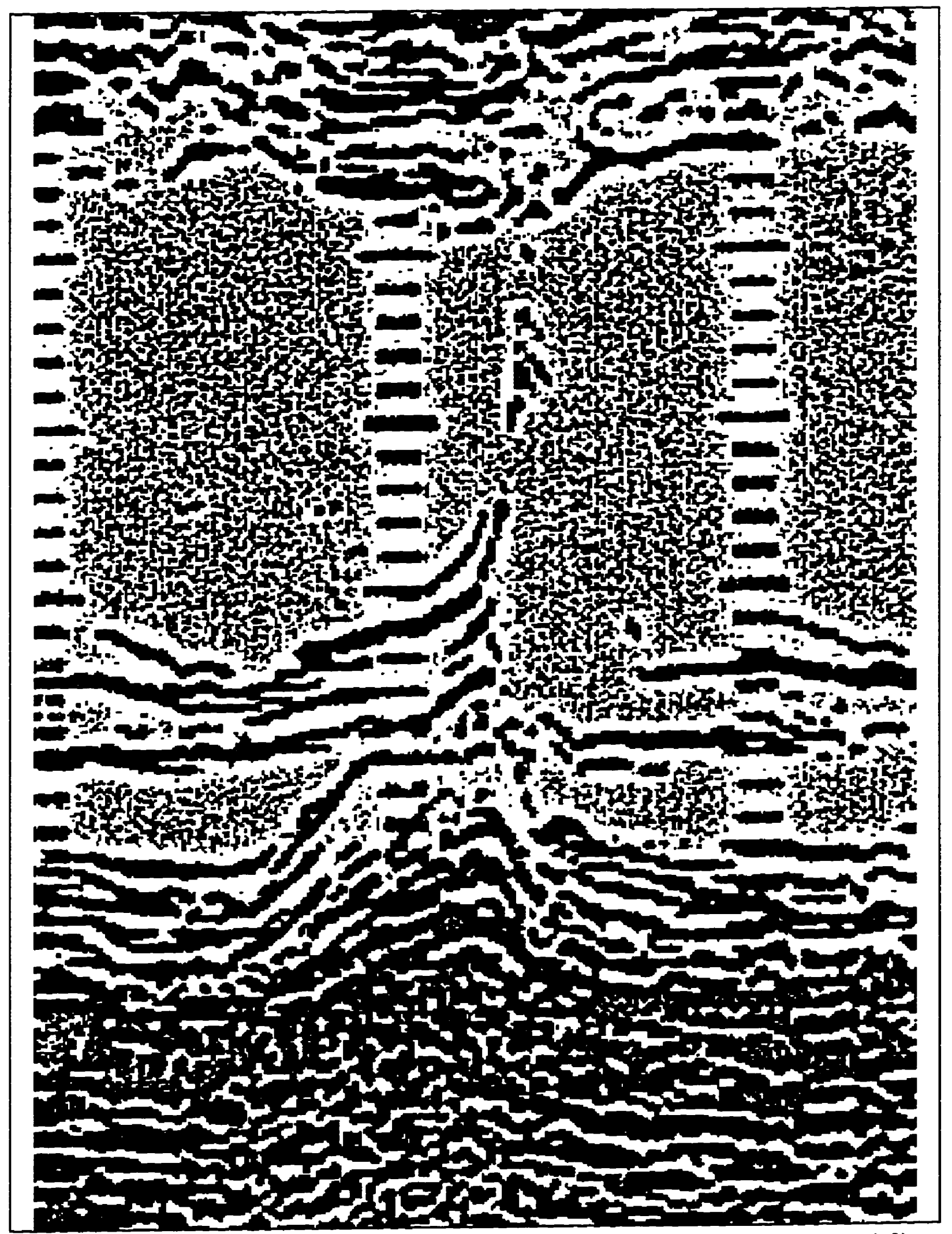

Figure 5-4 Black and White (+vel-ve regions) Result of LoG Filtering $(\sigma=1.0)$ 


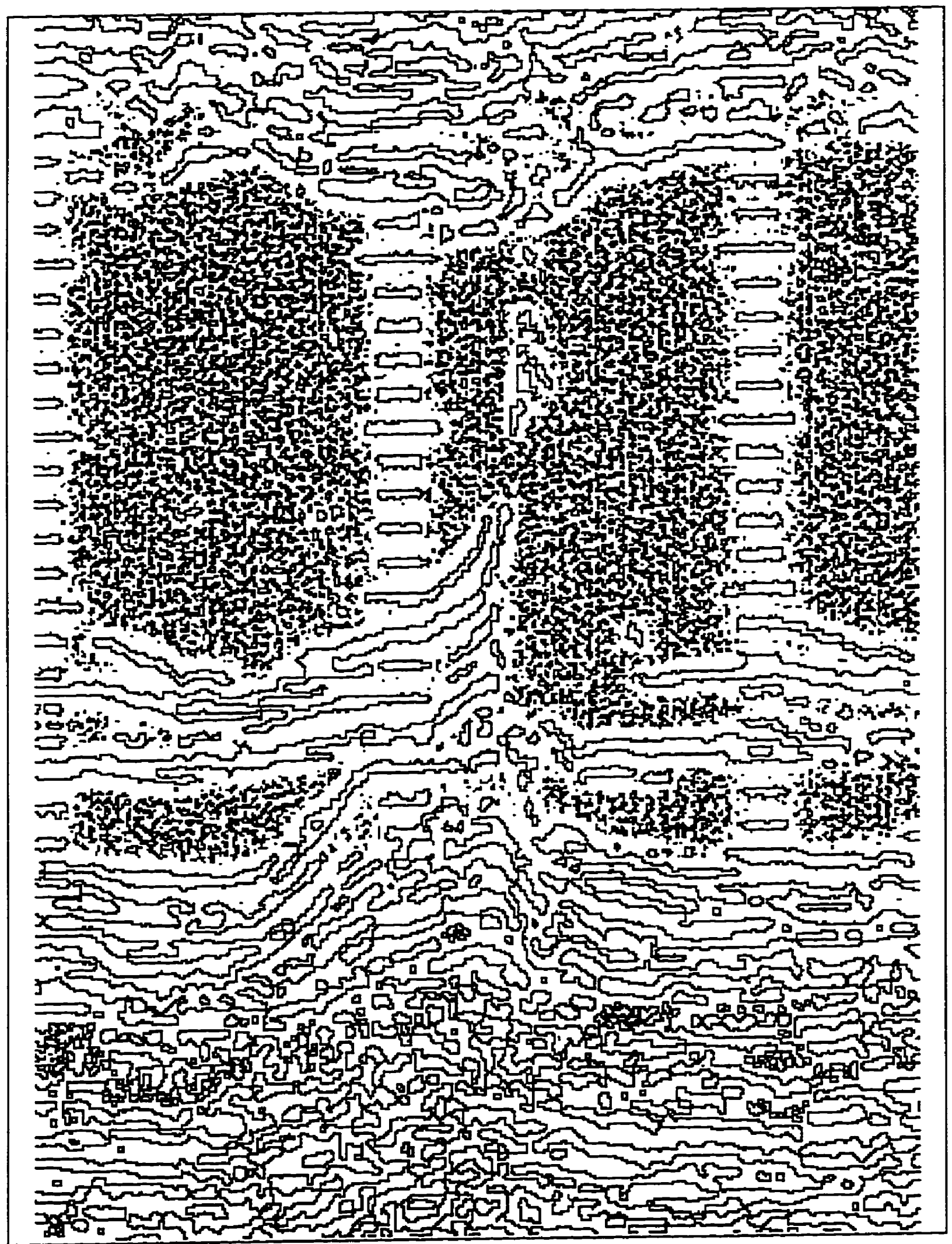

Figure 5-5 Edge Map Derived from LoG Filtering $(\sigma=1.0)$ 


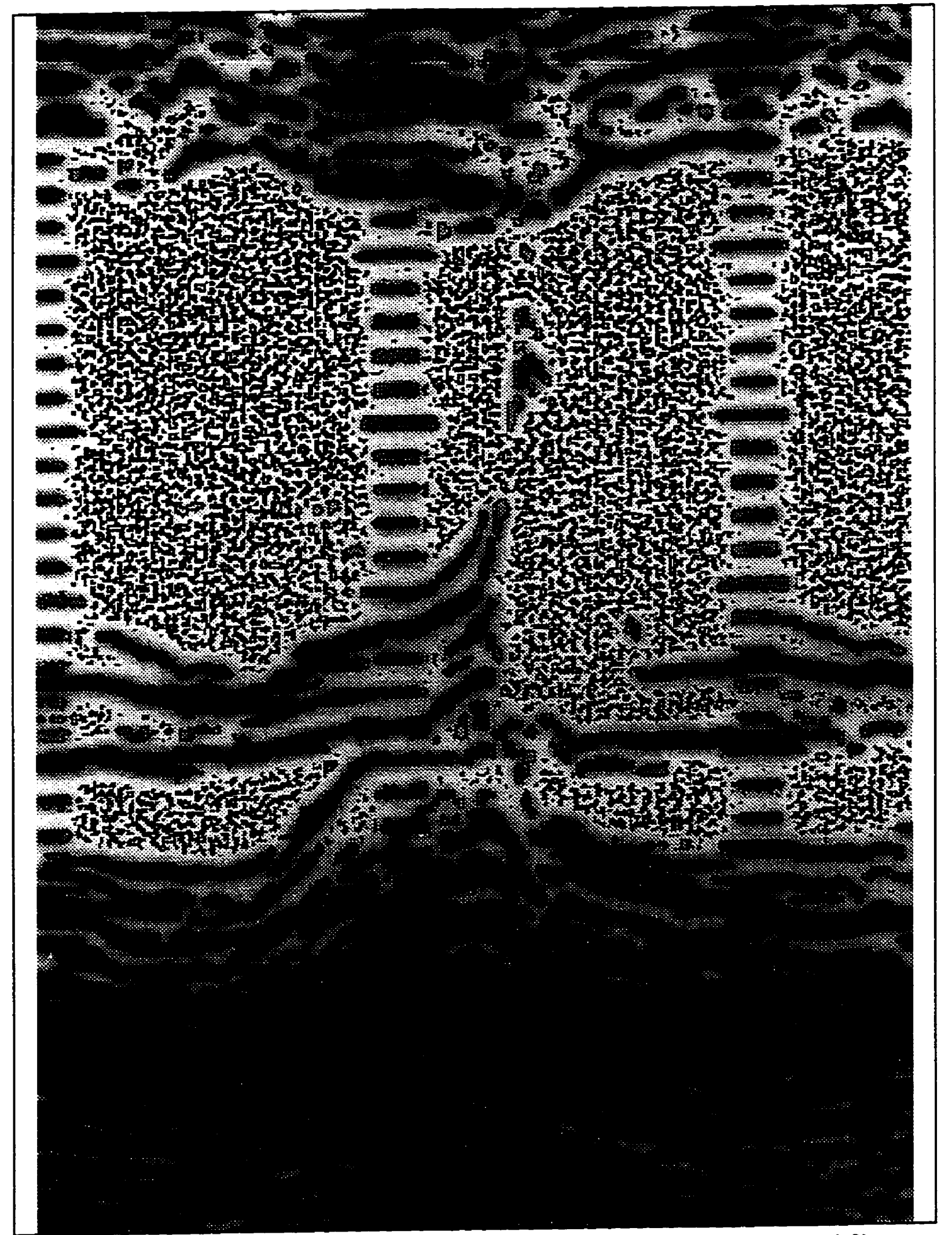

Figure 5-6 Edge Addition of Figure 5-5 to Original Test Image ( $\sigma=1.0)$ 


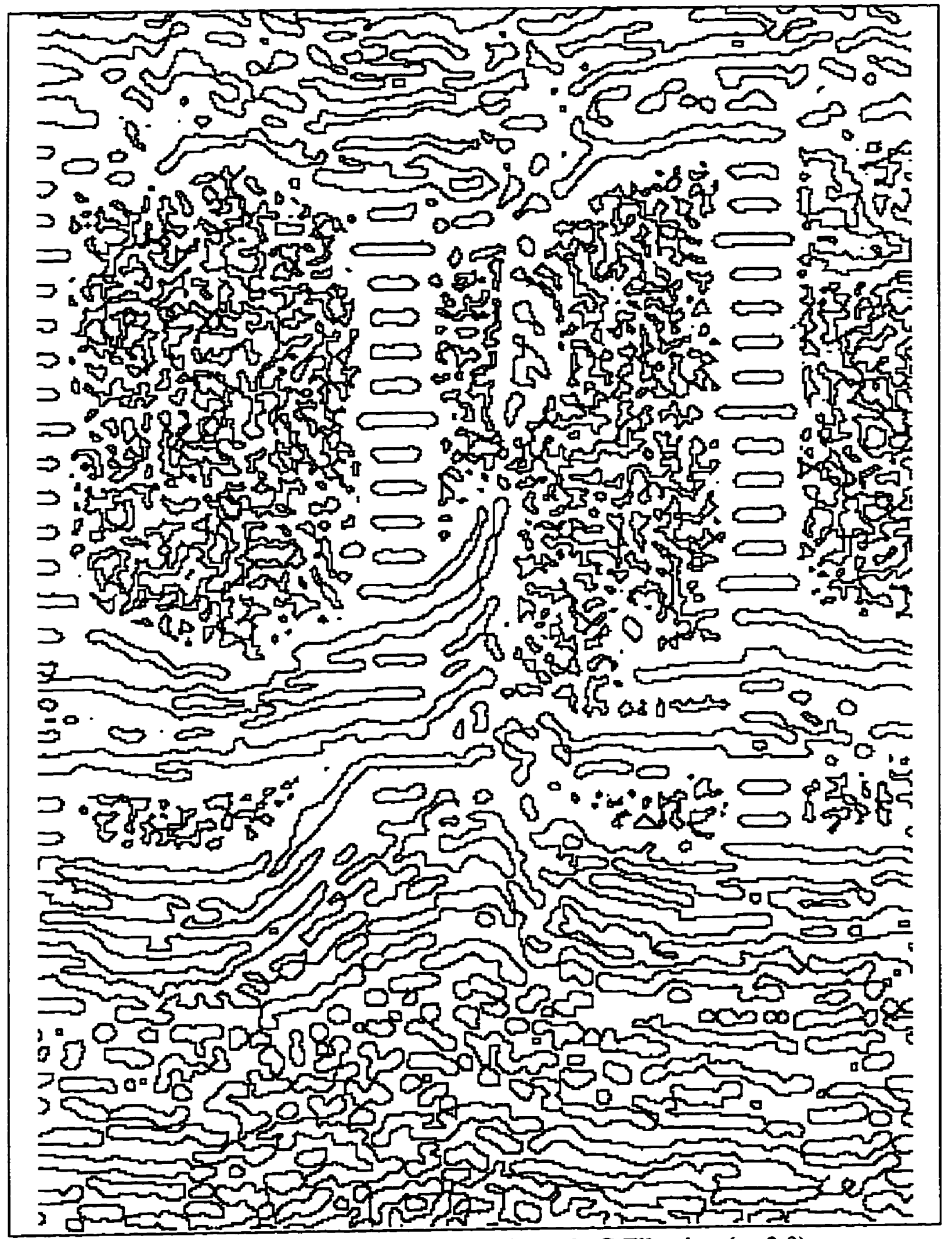

Figure 5-7 Edge Map Derived from LoG Filtering $(\sigma=2.0)$ 


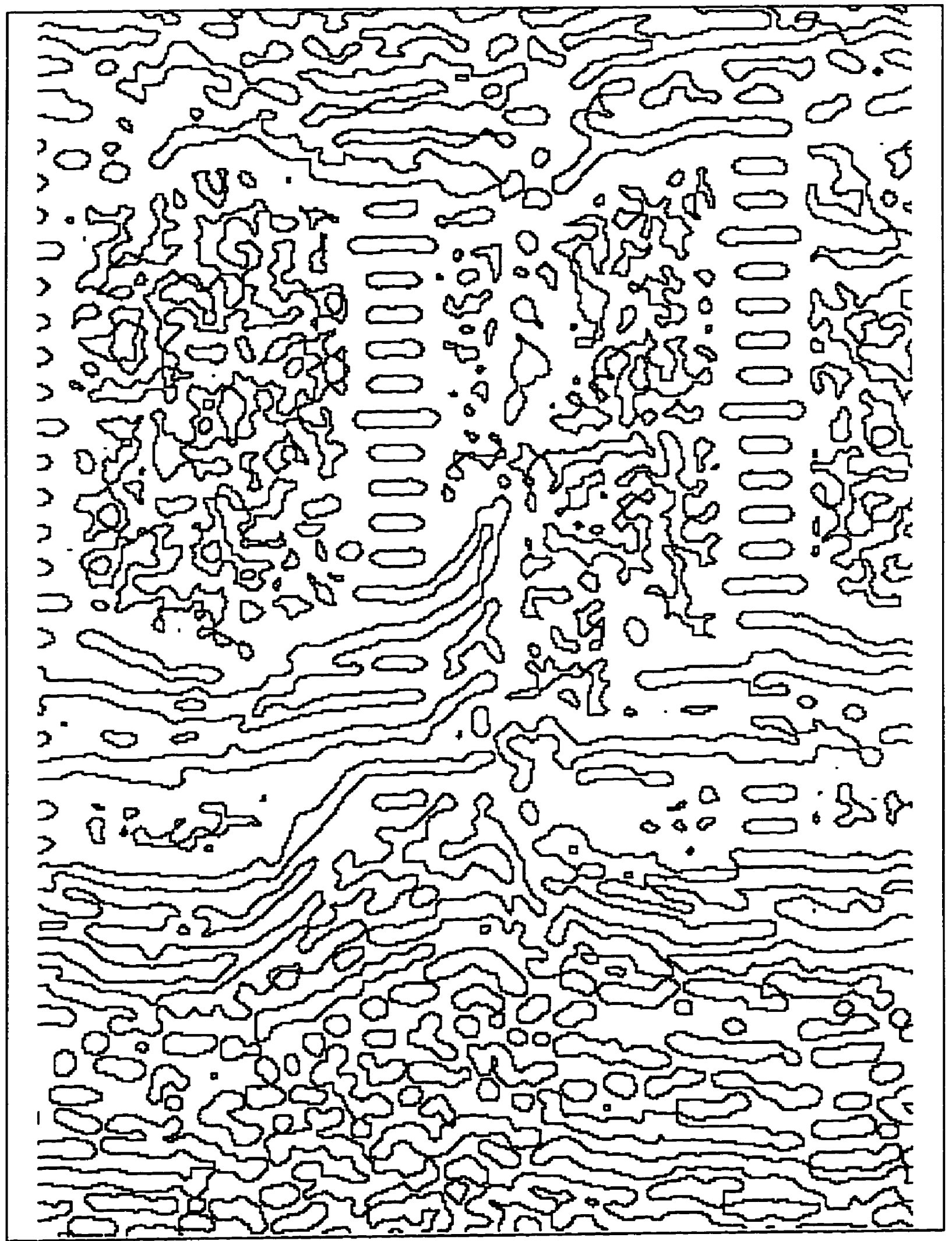

Figure 5-8 Edge Map Derived from LoG Filtering ( $\sigma=3.0)$ 


\subsection{Experimental Results Using the DoG Filter}

This section also presents a "slide show" of the results of DoG filtering on the test image. Figure 5-9 shows the result of applying the DoG filter with a $\sigma$ value of 0.5 . Note that the edges are displayed in varying gray scales where stronger contours are darker in colour. The next figure (5-10) shows all of the contours detected in black for comparison with the LoG results which were not weighted using a slope-of-zero-crossing or any other scheme. Figure 5-11 shows the result of thresholding figure 5-9 at a gray level of 20 to remove many of the weaker contours from the edge map. This reduces the number of edge points from approximately 75,000 to 30,000 . This reduced edge map is then added to the original image using edge addition (figure 5-12). Finally, to illustrate the effect of changing the $\sigma$ value with the DoG filter, figure 5-13 shows the edge map created when $\sigma=1.0$. Figure 5-14 shows figure 5-13 thresholded to black.

\subsection{Discussion/Comparison of Results}

It is difficult to compare the overall capabilities of one technique over another as the DoG filter results included a weighting for edges detected whereas the LoG results were not weighted. The resulting enhanced images shown in figures 5-6 and 5-12 illustrate this point quite readily as the DoG result is much clearer and provides much more information to an analyst due to the maintenance of edge strength differences within the image. Nonetheless, figures 5-5 and 5-14 provide a good means of comparing the gross number of edge points detected. The LoG approach produces approximately 67,000 points when $\sigma=1.0$ as opposed to 55,000 points for the DoG filter with the same resolution. This only indicates that the DoG filter is more selective in its determination of edge candidates; whether this makes it more accurate or not is 


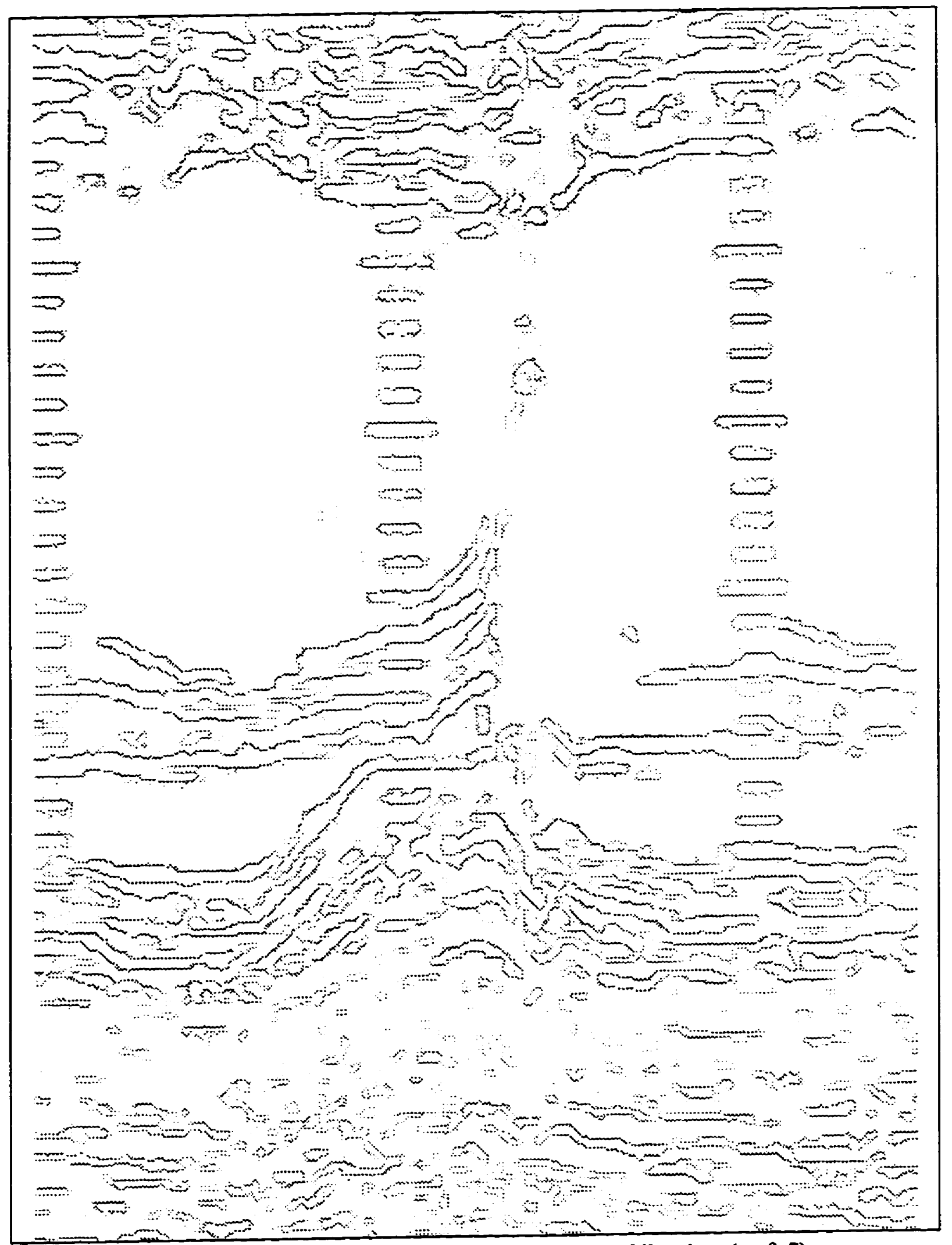

Figure 5-9 Edge Map Derived from DoG Filtering $(\sigma=0.5)$ 


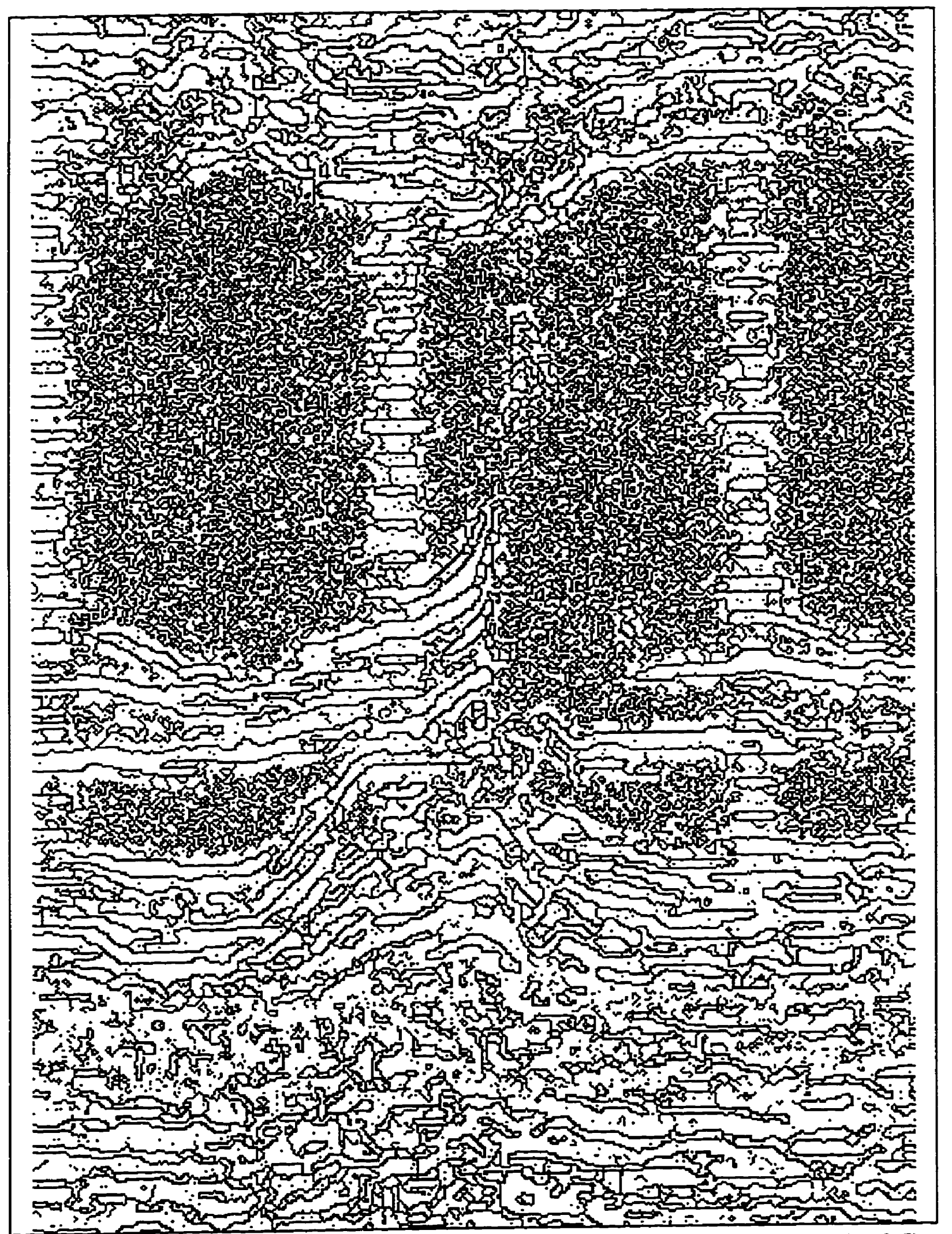

Figure 5-10 DoG Edge Map Thresholded with all Contours set to Black $(\sigma=0.5)$ 


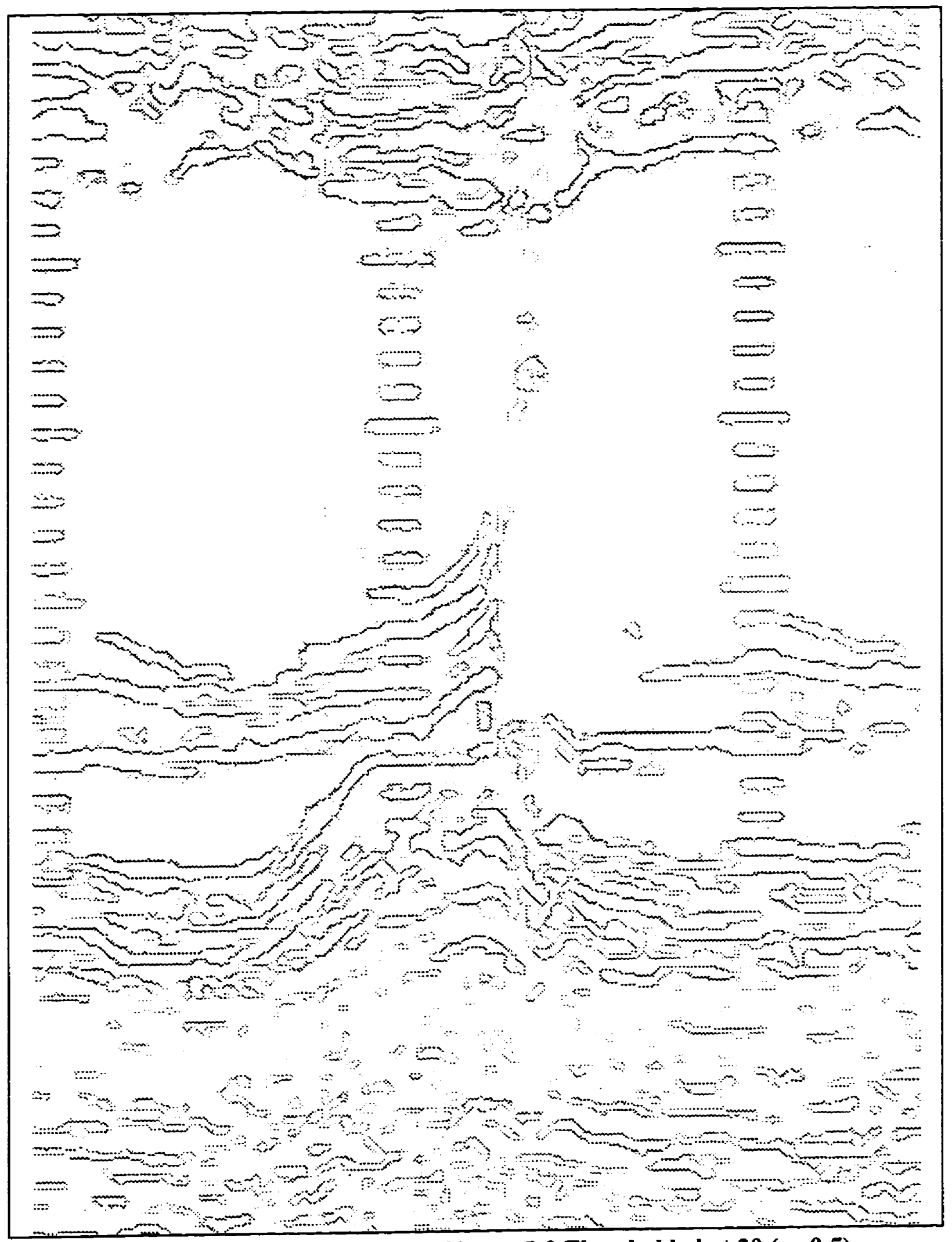

Figure 5-11 Edge Map from Figure 5-9 Thresholded at $20(\sigma=0.5)$ 


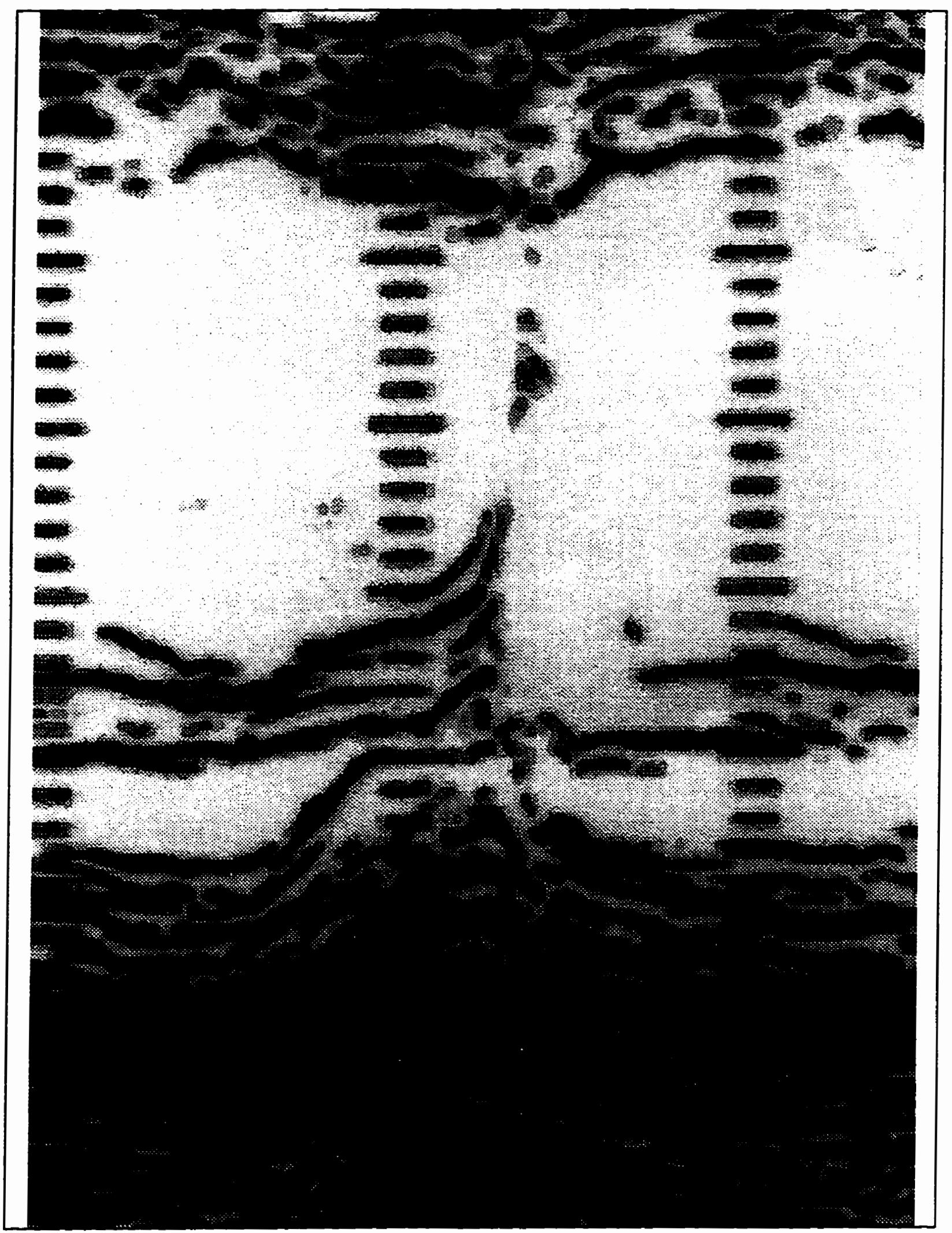

Figure 5-12 Addition of Figure 5-11 $(\sigma=0.5)$ to Original Image 


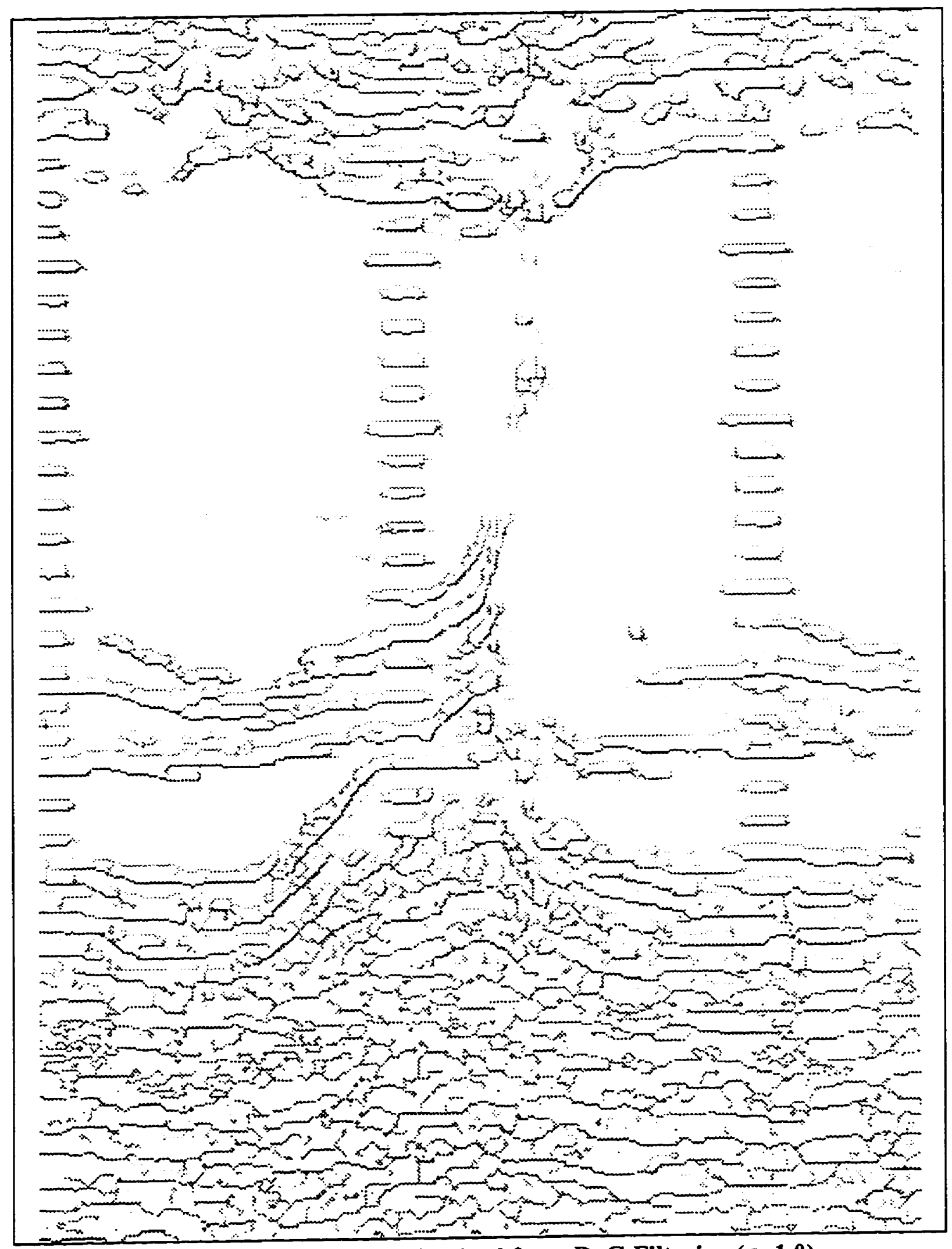

Figure 5-13 Edge Map Derived from DoG Filtering ( $\sigma=1.0)$ 


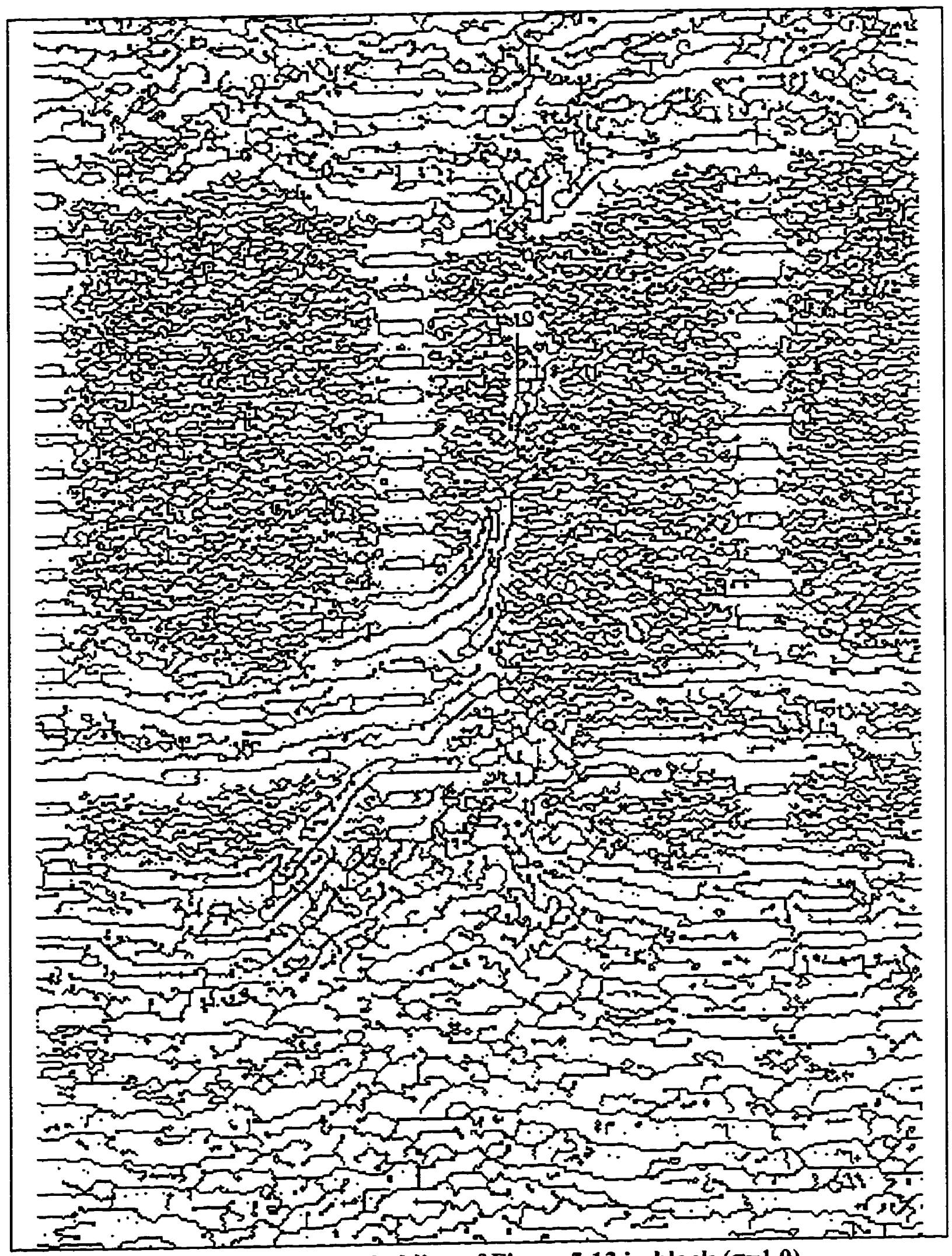

Figure 5-14 Thresholding of Figure 5-13 in black $(\sigma=1.0)$ 


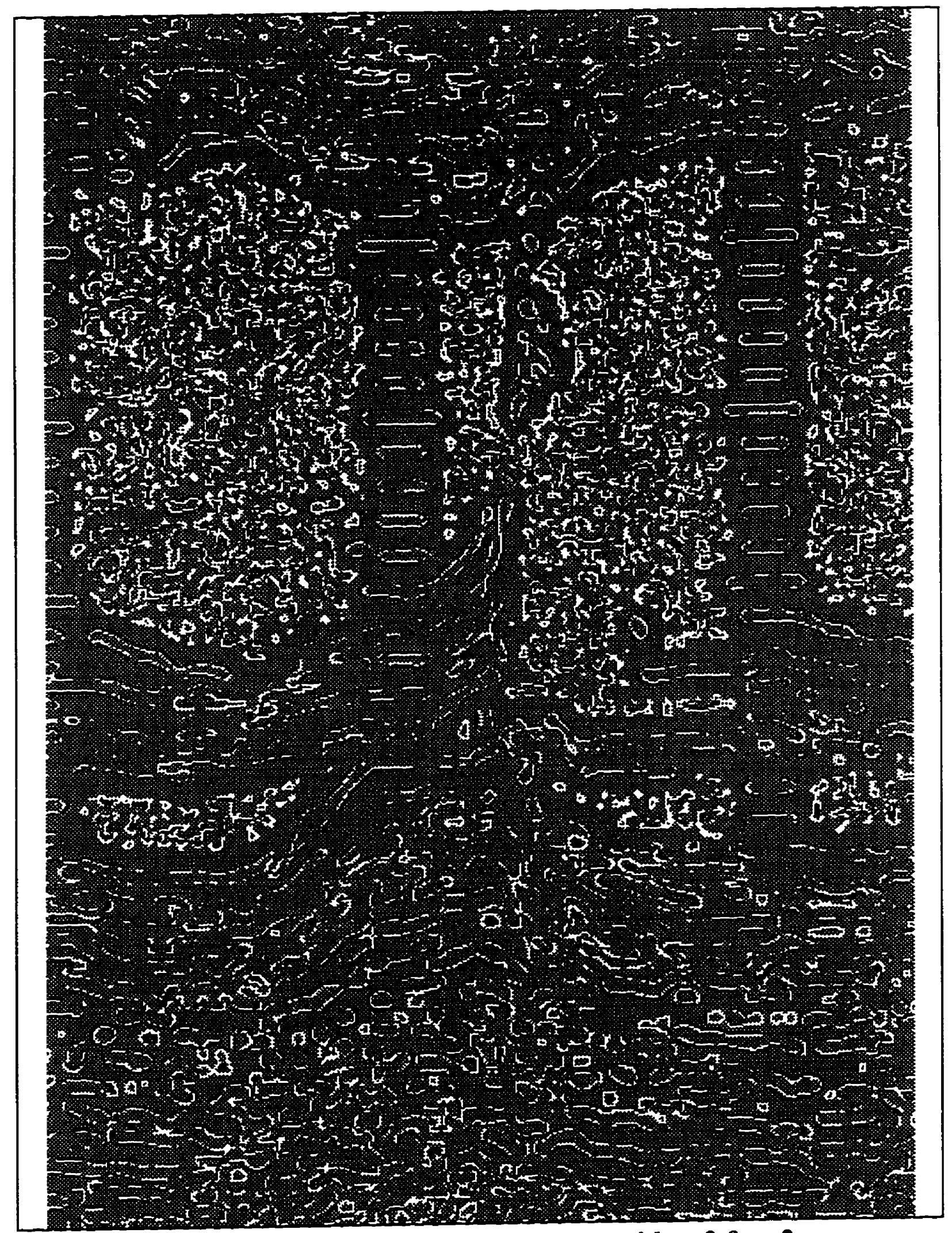

Figure 5-15 Subtraction of LoG Images with $\sigma=3 \& \sigma=2$ 
difficult to determine.

Another result which can be derived from the images presented is the effect of varying the $\sigma$, or resolution, factor. For both filters, increasing the size of the filter causes the original image to become more blurred. This can be seen by referring to the lower half of figures 5-5, 5-7 and 5-8 for the LoG filter and figures 5-9 and 5-13 for the DoG filter. It is possible to see how this affects the spatial accuracy of the processed image by subtracting the LoG edge map for $\sigma=3.0$ and $\sigma=2.0$. This result is shown in figure 5-15. The white lines are the edges found in $\sigma=2.0$ and the black lines are those for $\sigma=3.0$. This result appears to support the earlier contention that the loss of spatial accuracy is less than $1.4 \sigma$ when an image is smoothed with a Gaussian as part of the edge detection process as the pixels for $\sigma=2.0$ are not further than one pixel away from those of $\sigma=3.0$ (expected to be less than 1.4 pixels away). Further, the larger numbers of white pixels further support the contention that higher $\sigma$ values result in a greater loss of edge data.

Finally, the DoG enhanced image shown in figure 5-12 appears to be superior to the original (figure 5-1) in that edges are more sharply defined. There is no loss of information as the results of the edge detection process were merely added without normalization to the original image. It would, however, require objective analysis by trained professionals to confirm this hypothesis. Additionally, extensive testing on more heavily degraded images (higher noise levels) would help to validate the usefulness of this technique in actual diagnostic circumstances. 


\section{Chapter Six}

\section{Concluding Material}

\section{6-1 Introductory Comments}

This final chapter contains the concluding material for the thesis. It begins with a discussion of the major accomplishments of the thesis with regards to both of the stated aims:

1. To explore a wide range of aspects in low level vision including image storage. display, and enhancement; and

2. To develop a user-friendly software package capable of performing the functions studied.

The chapter ends with a discussion of possible areas of future research in the field of image processing.

\section{6-2 Summary of Thesis}

Chapters Two and Three of the thesis were intended to meet the first aim by providing a broad background of image processing concepts to the reader. In Chapter Two, the Tagged Image File Format was introduced as an example of one common technique to store digitized images. It was shown that the TIFF format is a powerful and dynamic tool for creating the link 
between the visual world and the digital environment of computers. This was followed by a brief discussion on M-mode Echocardiograms as the more advanced techniques explored in the thesis were applied to this field. A number of image processes were then presented which were broken down into four basic types: point, area, algebraic and geometric. All operations (algorithms) used in image processing fall into one of these four categories. The operations within any of these four groupings can be further broken down into one of two types of operations: unary and binary. Seven different types of unary operations (covering three types of processes) were presented within this chapter:
1. negation
2. brightness adjustment
(point process),
3. cropping
4. scaling
5. histogram equalization
(point process),
(geometric process),
6. histogram thresholding (geometric process), (point process),
7. median filtering (point process), and (area process).

The chapter ended with the presentation of three algorithms for binary operations which were all algebraic in nature:

1. addition,

2. subtraction, and

3. multiplication.

Chapter Three rounded off the examination of image processes by taking a detailed look at the area of image enhancement - specifically the detection of edges, or contours, within images. The chapter began with an discussion of some of the properties of edges within digitized images as well as a survey of many of the primary techniques which have been used to detect edges within digitized images over the past few decades. Two approaches were then selected from these to illustrate two different means of creating edge-maps for images. The first was 
based on the Laplacian of a Gaussian Filter as described by Dr F. Neycenssac in his article “Contrast Enhancement Using the Laplacian-of-a-Gaussian Filter" (1993). A detailed description of the algorithm employed and the mathematics behind it were provided as well as example results for the filter when applied to a small real-world image. To complete the chapter, the same was done for a second technique, the Derivative of a Gaussian Filter, based on a method described by Dr A. Korn in his article "Towards a Symbolic Representation of Intensity Changes in Images" (1988).

Chapter Four and Five were developed in response to the second aim of the thesis. Chapter Four provided a detailed description of a $C$ program which was written to implement each of the operations discussed in Chapter Two as well as the two edge detection techniques examined in Chapter Three. The program was designed using the top-down design approach and details of the conventions which were applied and the program layout were provided as a means of assisting the reader with following the layout and design of the coded routines. The processing environment was described next to introduce the reader to the interface that was developed to allow quick and easy access the various capabilities of the program. Several limitations of the equipment, code and the application associated with this environment were also provided. The chapter ended with an explanation of the various capabilities of the program for including importing images, "housekeeping" commands, delete commands, save commands and numerous processing commands.

Chapter Five demonstrated the capabilities of the program on a scanned image of an $\mathrm{M}$ mode Echocardiogram. The presentation was restricted to edge-detection; however, other capabilities were displayed or involved peripherally such as the list command (for TIFF 
information), histogram thresholding, addition and subtraction. To complete this chapter, the results of the application of the two edge detection schemes studied were then discussed and compared in general terms.

\section{6-3 Recommendations for Future Research}

The realm of image processing is vast and growing rapidly even as this research was being undertaken. Literally everyday, new advances in image processing areas such as animation, three-dimensional imaging, texture/motion analysis and pattern recognition are being made throughout the world. Concurrently, technologies for scanning, storing, manipulating and displaying digital images are being advanced equally quickly. This leaves an interested party with an almost overwhelming wealth of opportunities to choose from in this field. In regards to the specific areas examined within this work, a number of suggestions can be made for research intended to complement that which has been provided here. These include, but are certainly not limited to, the following:

1. An investigation of parallel processing to speed up the implementation of many of the processes selected, particularly the edge detection operations;

2. An analysis of other image storage formats such as GIF or PCX:

3. A subjective analysis of the results of the two edge detection schemes by specialists:

4. A study of techniques for automatically detecting structures derived from the implementation of the edge detectors on M-mode images (pattern matching/ pattern recognition);

5. The implementation of the LoG filter with a "slope of zero crossing" weight 
assignment scheme;

6. An analysis of the effects of changing the resolution of the edge detection filters described, sometimes called "focussing" [Berg87] within the literature: and

7. An investigation of other edge detection techniques such as those discussed in section 3-3.

Certainly, an interested reader will not find any shortage of material on any of these subjects. 


\section{References}

[Ak185] Akl Selim G, Parallel Sorting Algorithms, Academic Press Inc, Orlando, Florida. 1985.

[Akl89] Akl Selim G, The Design and Analysis of Parallel Algorithms, Prentice Hall, Englewood Cliffs, New Jersey, 1989.

[Alde63] Alder B et al, Methods in Computational Physics - Advances in Research and Applications, Vol 2, Academic Press, New York, 1963.

[Bank92] Banks Gary, "Masters Thesis: User Interfaces for Image Processing Systems." Kingston, Ontario: Royal Military College of Canada, 1992

[Berg84] Bergholm Fredrik, "Detection of Edges and Busy Regions Using Canny's Edge Detector," Royal Institute of Technology, Sweden, TRITA-NA-E8472, 1984.

[Berg87] Bergholm Fredrik, "Edge Focusing," IEEE Transactions on Pattern Analysis and Machine Intelligence, Vol PAMI-9, No 6, pp.726-741, 1987.

[Berz84] Berzins Valdis, “Accuracy of Laplacian Edge Detectors," Computer Vision. Graphics, and Image Processing, Vol 27, pp.195-210, 1984.

[Bor192-1] Borland C++ Library Reference: Runtime Library, Global Variables, CrossReference, Borland International Inc, Scotts Valley, California, 1992.

[Bor192-2] Borland C++User's Guide: Integrated Environment, Optimization, Command-Line Compiler, Installation, Borland International Inc, Scotts Valley, California, 1992.

[Borl92-3] Borland C++ Tools and Utilities Guide: Error Messages, Winsight, Make, Help/Resource Compilers, TLink, Borland International Inc, Scotts Valley, California, 1992.

[Bor192-4] Borland C++ Programmer's Guide: Language Structure, Class Libraries, Advanced Programming Techniques, ANSI C Implementation, Borland International Inc, Scotts Valley, California, 1992.

[Buf90] Du Buf JMH, "Gabor Phase in Texture Discrimination," Signal Processing, Vol 21. pp. 221-240, 1990.

[Cann83] Canny John, "Technical Report 720: Finding Edges and Lines in Images," Cambridge Massachusetts: MIT Artificial Intelligence Laboratory, 1983. 
[Cann86] Canny John, "A Computational Approach to Edge Detection", IEEE Transactions on Pattern Anaiysis and Machine Intelligence, Vol PAMI-8, No 6, pp. 679-698, 1986.

[Carl87] Carlotto Mark J, "Histogram Analysis Using a Scale-Space Approach," IEEE Transactions on Pattern Analysis and Machine Intelligence, Vol PAMI-9, No 1. pp. 121-129, 1987.

[Cast79] Castleman Kenneth R, Digital Image Processing, Prentice Hall, Englewood Cliffs. New Jersey, 1979.

[Chen89] Chen JS and Medioni G, "Detection, Localization, and Estimation of Edges," IEEE Transactions on Pattern Analysis and Machine Intelligence, Vol 11, No 2, pp.191$198,1989$.

[Chu88] Chu C Henry, "Detecting Left Ventricle Endocardial and Epicardial Boundaries by Digital Two-Dimensional Echocardiography," IEEE Transactions on Medical Imaging, Vol 7, No 2, pp. 81-90, 1983.

[Coll87] Collins Steve $\mathrm{M}$ et al, "Computer-Assisted Edge Detection in Two-Dimensional Echocardiography: Comparison with Anatomic Data," The American Journal of Cardiography, Vol 53, 1987.

[DaPo88] DaPonte JS and Fox MD, "Enhancement of Chest Radiographs with Gradient Operators," IEEE Transactions on Medical Imaging, Vol 7, No 2, pp. 109-11 7. 1988.

[Deri93] Deriche R and Giraudon G, "A Computational Approach for Corner and Vertex Detection," International Journal of Computer Vision, Vol 10, No 2, pp.101-124. 1993.

[Doug94] Dougherty Edward R, Digital Image Processing Methods, Marcel Dekker Inc. New York, New York, 1994.

[Dume79] Dumesnil JG, Shoucri RM, Laurenceau JL and Turcot J: "A mathematical model of the dynamic geometry of the intact left ventricle and its application to clinical data." Circulation 59, pp. 1024-1034, 1979.

[Ekst84] Ekstrom Michael P, Digital Image Processing Techniques, Academic Press Inc, Orlando, Florida, 1984.

[Fais91] Faison Edmund W Jr, Graphical User Interfaces with Turbo C++, Sams, A Division of Macmillan Inc, Carmel, Indiana, 1991.

[Free91] Freeman WT and Adelson EH, "The Design and Use of Steerable Filters," IEEE Transactions on Pattern Analysis and Machine Intelligence, Vol 13, No 9, pp.891906, 1991.

[Galb90] Galbiati Louis J Jr., Machine Vision and Digital Image Processing Fundamentals, Prentice Hall, Englewood Cliffs, New Jersey, 1990. 
[Gonz79] Gonzalez Rafael C and Wintz Paul, Digital Image Processing, Addison-Wesley Publishing Company, Reading Massachusetts, 1979.

[Grim85] Grimson WEL and Hildreth EC, "Comments on "Digital Step Edges from Zero Crossing of Second Directional Derivatives"," IEEE Transactions on Pattern Analysis and Machine Intelligence, Vol PAMI-7, No 1, pp. 121-129, 1985.

[Guid88] Guiducci Antonio, "Comer characterization by differential geometry techniques," Pattern Recognition Letters, Vol 8, pp.311-318, 1988.

[Hara83] Haralick Robert M, "Ridges and Valleys on Digital Images," Computer Vision. Graphics, and Image Processing, Vol 22, pp. 28-38, 1983.

[Hara84] Haralick Robert M, "Digital Step Edges from Zero Crossing of Second Directional Derivatives," IEEE Transactions on Pattern Analysis and Machine Intelligence, Vol PAMI-6, No 1, pp. 58-68, 1984.

[Hart85] Hartley Ralph, "A Gaussian-Weighted Multiresolution Edge Detector", Computer Vision, Graphics, and Image Processing, Vol 30, pp.70-83, 1985.

[Hext90] Hext Jan, Programming Structures - Volume I Machines and Programs, Prentice Hall Canada Inc, Toronto, 1990.

[Hild83] Hildreth Ellen C, "The Detection of Intensity Changes by Computer and Biological Systems," Computer Vision, Graphics, and Image Processing, Vol 22, pp. 1-27, 1983.

[John87] Johnson Nelson, Advanced Graphics in C: Programming and Techniques, McGrawHill Inc, Berkeley, Califormia, 1987.

[Kana80] Kanade Takeo, "Region Segmentation: Signal vs Semantics," Computer Vision. Graphics, and Image Processing, Vol 13, pp.279-297, 1980.

[Kern 78] Kernighan BW and Ritchie DM, The C Programming Language, Prentice-Hall Inc. Englewood Cliffs, New Jersey, 1978.

[Korn88] Korn Axel F, 'Towards a Symbolic Representation of Intensity Changes in Images.' IEEE Transactions on Pattern Analysis and Machine Intelligence, Vol 10, No 5, pp. 610-625, 1988.

[Laur81] Laurenceau $\mathrm{JL}$ and Malergue MC, The Essentials in Echocardiography, Martinus Nijhoff Publishers, The Hague, The Netherlands, 1981.

[Law96] Law Todd, "Image Filtering, Edge Detection, and Edge Tracing Using Fuzzy Reasoning," IEEE Transactions on Pattern Analysis and Machine Intelligence, Vol 18, No 5, pp. 481-491, 1996. 
[Liaw95] Liaw Wilson MacGyver, Reading GIF Files, Dr. Dobb's Journal, pp. 56, 58, 60. 103. 106-107.

[Lind91] Lindley Craig A, Practical Image Processing in C : Acquisition, Manipulation and Storage, John Wiley \& Sons, Inc, New York, 1991.

[Ling79] Linger Richard C et al, Structured Programming: Theory and Practice, AddisonWesley Publishing Company, Massachusetts, 1979.

[Luns86] Lunscher WHHJ and Beddoes MP, "Optimal Edge Detector Design I: Parameter Selection and Noise Effects," IEEE Transactions on Pattern Analysis and Machine Intelligence, Vol PAMI-8, No 2, pp. 164-177, 1986.

[Luse93] Luse Marv, Bitmapped Graphics Programming in C++, Addison-Wesley Publishing Company, Massachusetts, 1993.

[Mach83] Machuca R and Phillips K, "Applications of Vector Fields to Image Processing," IEEE Transactions on Pattern Analysis and Machine Intelligence, Vol PAMI-5. No 3. pp. 316-329, 1983.

[Marr80] Marr D and Hildreth E, "Theory of Edge Detection," Proceedings Royal Society of London, B207, pp. 187-217, 1980.

[Marr82] Marr David, Vision, WH Freeman and Company, San Francisco, 1982.

[Mehr90] Mehrotra R and Nichani S, "Corner Detection," Pattern Recognition, Vol 23. No Il. pp.1223-1233, 1990.

[Mehr92] Mehrotra R et al., "Gabor Filter-Based Edge Detection," Pattern Recognition, Vol 25. No 12, pp. 1479-1494, 1992.

[Mill87] Miller PL and Miller LW, Programming by Design - A First Course in Structured Programming, Wadsworth Publishing Company, Belemont, Califomia, 1987.

[Mull90] Mullin Mark, Object Oriented Program Design - With Examples in C++, AddisonWesley Publishing Company Inc, Massachusetts, 1990.

[Murr94] Murray Mike, "Masters' Thesis: Constraint Programming Applied to a Scheduling Problem," Kingston, Ontario: Royal Military College of Canada, 1994.

[Namu94] Namuduri KR et al, "Efficient Computation of Gabor Filter Based Multiresolution Responses," Pattern Recognition, Vol 27, No 7, pp. 925-938, 1994.

[Neyc93] Neycenssac Franck, "Contrast Enhancement Using the Laplacian-of-a-Gaussian Filter," CVGIP: Graphical Models and Image Processing, Vol 55, No 6, pp. 447-463, 1993. 
[Nibl86] Niblack Wayne, An Introduction to Digital Image Processing, Prentice Hall International (UK) Ltd, Hertfordshire, Denmark, 1986.

[Niem87] Nieminen Ari et al, "A New Class of Detail-Preserving Filters for Image Processing." IEEE Transactions on Pattem Analysis and Machine Intelligence, Vol PAMI-9. No 1, pp.74-90, 1987.

[Papo80] Papoulis Athanasios, Circuits and Systems - A Modern Approach, Holt, Rinehart and Winston Inc, Mew York, 1980.

[PCom1] Personal communications with Dr. Rachad Shoucri, Professor, Department of Mathematics and Computer Science, Royal Military College of Canada, Kingston. Ontario, I June 95 to 28 July 96.

[PCom2] Personal communications with Major J.A.M. Poliquin, Canadian Forces Communication and Electronics Branch and Assistant Professor, Royal Military College of Canada, Kingston, Ontario, 1 June 95 to 15 June 1996.

[PCom3] Personal communications with Dr. Mike Wilmut, Professor, Department of Mathematics and Computer Science, Royal Military College of Canada, Kingston. Ontario, 1 September 95 to 15 June 96.

[Pel193] Pelle G and Belamari MY, "Microcomputer-based system for automatic analysis of M-mode Echocardiograms," Journal of Biomedicine Eng, Vol 15, pp.274-278, 1993.

[Ruiz95] Ruiz EES and Fairhurst MC, "Improved approach to boundary location in twodimensional echocardiographic images," IEE Proceedings - Visual Image Signal Processing, Vol 142, No 3, pp. 121-127, 1995.

[Sark91] Sarkar S and Boyer KL, "On Optimal Infinite Impulse Response Edge Detection Filters," IEEE Transactions on Pattern Analysis and Machine Intelligence, Vol 13. No 11, pp.1154-1171, 1991.

[Shen95] Shen J and Shen W, "Image Smoothing and Edge Detection by Hermite Integration," Pattern Recognition, Vol 28, No 8, pp.1159-1166, 1995.

[Simo76] Simon Jean Claude and Rosenfeld Azriel, Digital Image Processing and Analvsis. Noordhoff International Publishing, Netherlands, 1976.

[Tadr95] Tadrous Paul J, "A Simple and Sensitive Method for Directional Edge Detection in Noisy Images," Pattern Recognition, Vol 28, No 10, pp.1575-1586, 1995.

[Taga90] Tagare HD and deFigueiredo RJP, "On the Localization Performance Measure and Optimal Edge Detection," IEEE Transactions on Pattern Analysis and Machine Intelligence, Vol 12, No 12, pp.1186-1190, 1990.

[Talt85] Talton David, "Implementation of a Gaussian-Smoothing Gradient-Edge Detector," University of Pennsylvania, MS-CIS-85-12, 1985. 
[Tond85] Tondo CL and Gimpel SE, The C Answer Book, Prentice-Hall Inc, Englewood Cliffs, New Jersey, 1985.

[Torr86] Torre V and Poggio TA, "On Edge Detection," IEEE Transactions on Pattern Analysis and Machine Intelligence, Vol PAMI-8, No 2, pp. 147-163, 1986.

[Tuai85] Tuai Gregory, "Masters' Thesis: Development of an Image Processing Technique to Enhance Cardiac Wall Structures in Medical Ultrasound Images," Washington DC: University of Washington, 1985.

[Vett93] Vetterling WT, Teukolsky SA, Press WH and Flannery BP, Numerical Recipes Example Book (C), Press Syndicate of the University of Cambridge. New York. 1993.

[Wate95] "Graduate Thesis Regulations," University of Waterloo, Waterloo, Ontario, 1995.

[Will90] Williams DJ and Shah M, "Edge Contours Using Multiple Scales," Computer Vision. Graphics, and Image Processing, Vol 51, pp.256-274, 1990.

[Xin95] Xin Kai et al, “A Scale-space Filtering Approach for Visual Feature Extraction," Pattem Recognition, Vol 28, No 8, pp.1145-1158, 1995. 


\section{Appendix A}

\section{List of C++ Drivers}

The following is a list of the drivers that are required to run the program:

1. bgiobj.exe

2. bold.chr

3. egavga.bgi

4. bold.chr and

5. goth.chr

The following is a list of drivers that may be required to run the program if the system being used is not EGA/VGA:
1. att.bgi
2. cga.bgi
3. herc.bgi
4. ibm8514.bgi and
5. pc3270.bgi

Finally, the following drivers may be required for users who add code to the existing program which involves text being displayed in a font other than the two currently used by the program:
1. euro.chr
2. Icom.chr
3. litt.chr
4. sans.chr
5. scri.chr
6. simp.chr
7. trip.chr
8. tscr.chr

and 


\section{Appendix B}

\section{List of $\mathrm{C}++$ Libraries Used by the Program}

The following is a list of the $\mathrm{C}++$ libraries which are currently used by the application program:

1. alloc.h

2. conio.h

3. dir.h

4. dos.h

5. graphics.h

6. math.h

7. stdio.h

8. stdlib.h and

9. string.h

As code is added to the program in the future, a programmer must be certain to include any new libraries required by any new $\mathrm{C}++$ library routines [Borl92-1] which are incorporated. These libraries can be included with an "include" statement at the beginning of the new routine or simply be added to the file "c_libs.h" which is a master library file created for the program. 


\section{Appendix C}

\section{Program Image Processing Routines}

The following is a list of each of the image processing operations which can be executed by the program and their applicable function names within the application program. Also included is a page reference for where the theory for each process is provided within the thesis.

\section{Operation}

Addition of two images

Brightness adjustment:

Crop

Flip

Filters:

Histogram Operations:

Multiply two images

Negate an image

Subtract two images

Scale an image
Function Name

ADDO

BRIGHTNESS()

BRIGHTNESS()

CROPO

FLIP()

BLURO

DOGO

LOG()

MEDIANO

HISTOGRAMO

HISTOGRAMO

EQUALIZEO

MULTIPLYO

NEGATEO

SUBTRACT0

ZOOMO

\section{Page Reference}

\section{$31-32$}

24

25

25

23

39

58-73

48-58

31

27

27-28

28

33

24

$32-33$

26 


\section{Appendix D}

\section{List of Common Tags and Data Types Used by TIFF}

The following is a list of data types used by TIFF:

$\begin{array}{cll}\text { data type } & \text { size (in bytes) } & \text { description } \\ 1 & 1 & \text { BYTE } \\ 2 & 1 & \text { ASCII code } \\ 3 & 2 & \text { unsigned SHORT integer } \\ 4 & 4 & \text { unsigned LONG integer } \\ 5 & 8 & \text { two LONGs, the first is the numerator of } \\ & \text { the fraction, the second is the denominator }\end{array}$

These data types are used by IFD entries to identify the type of data to which a tag is referring. The count field indicates the number of such data types that the tag requires to complete its description. Thus the data_length for the tag's information is

$$
\text { data_length }=\text { count } * \text { sizeof }(\text { type }) \text {. }
$$

If the data_length is less than four bytes, the tag's information is contained within the IFD entry's valofs field. Otherwise, this field points to the starting location (offset from the beginning of the TIFF file) of the tag's information. The length of the consecutive string of bytes containing the information in this case is length as defined above. The following is a list of tags, and their data types, which are commonly found with TIFF files:

\begin{tabular}{||l|l|l|l|}
\hline \multicolumn{2}{|c|}{ Tags } & \multicolumn{1}{c|}{ Type } & Meaning \\
\hline Decimal & Hexadecimal & \multicolumn{1}{|c|}{} \\
\hline 254 & FE & LONG & new subfile type \\
\hline 256 & 100 & SHORT / LONG & image width \\
\hline
\end{tabular}




\begin{tabular}{||l|l|l|l|}
\hline \multicolumn{2}{|c|}{ Tags } & \multicolumn{1}{|c|}{ Type } & \multicolumn{1}{|c|}{ Meaning } \\
\hline Decimal & Hexadecimal & \multicolumn{1}{|c||}{} \\
\hline 257 & 101 & SHORT / LONG & image length (height) \\
\hline 258 & 102 & SHORT & bits per sample \\
\hline 259 & 103 & SHORT & compression \\
\hline 262 & 106 & SHORT & photometric interpretation \\
\hline 270 & 10 & ASCII & image description \\
\hline 271 & $10 \mathrm{~F}$ & ASCI & make of scanner or digitizer used \\
\hline 272 & 110 & ASCU & model of scanner or digitizer used \\
\hline 273 & 111 & SHORT / LONG & strip offsets \\
\hline 277 & 115 & SHORT & samples per pixel \\
\hline 278 & 116 & SHORT / LONG & rows per strip \\
\hline 279 & 117 & SHORT / LONG & strip byte counts \\
\hline 282 & $11 \mathrm{~A}$ & RATIONAL & x resolution \\
\hline 283 & $11 \mathrm{~B}$ & RATIONAL & y resolution \\
\hline 290 & 122 & SHORT & gray response unit \\
\hline 291 & 123 & SHORT & gray response curve \\
\hline 296 & 128 & SHORT & resolution unit \\
\hline 301 & $12 \mathrm{D}$ & SHORT & colour response curve \\
\hline 305 & 131 & ASCII & name and release number of software used to create \\
\hline 306 & 132 & ASCII & date and time of image creation \\
\hline 315 & $13 \mathrm{~B}$ & ASCII & image's creator \\
\hline 320 & 140 & SHORT & colour map \\
\hline
\end{tabular}

For more information on these and other tags, see [Lind91] Appendix 2. 